\title{
LAND OF RETREATING GLACIERS - CHECKLIST OF LIVERWORTS ON SVALBARD
} СТРАНА ОТСТУПАЮЩИХ ЛЕДНИКОВ - ЧЕКЛИСТ ПЕЧЕНОЧНИКОВ СВАЛЬБАРДА

\author{
LARS SÖDERSTRÖM ${ }^{1}$, NADEZHDA A. KONSTANTINOVA ${ }^{2}$, ANDERS HAGBORG ${ }^{3}$, TOMMY \\ PREST $^{4}$, KRISTIAN HASSEL ${ }^{4} \&$ MATT VON KONRAT ${ }^{3}$ \\ ЛАРС СЁДЕРСТРЁМ ${ }^{1}$, НАДЕЖДА А. КОНСТАНТИНОВА ${ }^{2}$, АНДЕРС ХАГБОРГ ${ }^{3}$, ТОММЫ ПРЕСТЁ ${ }^{4}$, \\ КРИСТИАН ХАССЕЛЬ ${ }^{4}$, МАТТ ФОН КОНРАТ ${ }^{3}$
}

Abstract

\begin{abstract}
The checklist of liverworts of the Svalbard archipelago contains all published data on the occurrence of liverworts in the Svalbard archipelago. The list includes 105 species, five more reported species that only doubtfully occur there, and 51 species previously listed for the archipelago that are excluded from Svalbard. In addition, the locations of 67 species new to different regions of the archipelago are reported. Two species, Neoorthocaulis hyperboreus and Riccardia chamedryfolia, are listed as new to the archipelago. Two old names are typified and synonymized with taxa common on Svalbard.

Резюме

Чек лист печеночников Свальбарда обобщает все опубликованные данные о распространении видов на архипелаге. Список включает 105 видов, находки еще пяти видов представляются сомнительными, а 51 вид, приводившийся ранее для архипелага исключен из состава флоры. Для 67 видов сообщается о находках, которые являются новыми для отдельных регионов архипелага. Два вида, Neoorthocaulis hyperboreus и Riccardia chamedryfolia, приводятся как новые для Свальбарда. Типифицированы два старых названия таксонов, которые отнесены к распространенным видам.
\end{abstract}

KEYWORDS: Checklist, Hepaticae, Svalbard, New reports

\section{GENERAL INTRODUCTION}

Svalbard is situated between $74^{\circ}$ to $81^{\circ} \mathrm{N}$ within the Arctic. It was first sighted by the Dutch Willem Barentsz in 1596, although there are indications that Vikings and/ or Pomors may have been there before. In the earliest times Svalbard was mostly visited for whale hunting. Smeerenburg was one of the first settlements, established by the Dutch in 1619, and served as a main hub for whaling, but was abandoned already in 1660. Several other temporary whaling / hunting settlements existed but by mid-19th century whaling was basically over (Arlov 1994).

Russian Pomors were active as hunters from the mid $16^{\text {th }}$ century with a peak period during the $18^{\text {th }}$ century. Unlike whaling, this activity was sustainable as they moved around. Today there are many remnants of this period (Arlov 1994).

Coal mining started in the beginning of the 20th century. Several settlements were established by various nations around coal mines. One of the largest, Pyramiden, was founded by the Swedish but sold to the Soviet Union. At most
1200 persons lived there but it was abandoned in 1998 .

Although there have been many settlements of various duration and for various reasons, today only four permanent settlements exist. The two commercial centres are Longyearbyen, the main settlement with the international airport and about 2400 inhabitants, and Barentsburg, the main Russian settlement with some 400 inhabitants. In addition, there are two year-around manned research centres, Ny-Ålesund, the large research centre hosting several nations research institutions, and Isbjørnhamna at Hornsund, the main Polish research centre. Norway also has two additional year-around manned weather stations, Bjørnøya and Hopen.

Politically, the archipelago belongs to Norway, but through the Svalbard Treaty of 1920 all signatory countries have right to perform commercial activities (today mainly coal mining and tourism). Sometimes Jan Mayen is included in the concept of Svalbard (mainly based on the unfortunate ISO code SJ, "Svalbard and Jan Mayen") but this is neither politically nor geographically motivated.

1 - Norwegian University of Science and Technology, Department of Biology, N-7491 Trondheim, Norway; email: lars.soderstrom@ntnu.no; ORCID: 0000-0002-9315-4978

2 - Polar-Alpine Botanical Garden, Kola Sci. Centre, Russ. Acad. Sci., Kirovsk-6, Murmansk Province, 184256 Russia E-mail: nadya50@list.ru; ORCID 0000-0002-7600-0512

3 - Field Museum of Natural History, Chicago, Illinois, 60605 USA; email: (MK) mvonkonrat@fieldmuseum.org, (AH) ahagborg@fieldmuseum.org; ORCID (MK): 0000-0001-9579-5325, (AH) 0000-0001-8390-319x

4 - Norwegian University of Science and Technology, University Museum, N-7491 Trondheim; email: (KH) kristian.hassel@ntnu.no, ORCID: 0000-0002-1906-8166; (TP) tommy.presto@ntnu.no; ORCID 0000-0003-3770-6296 


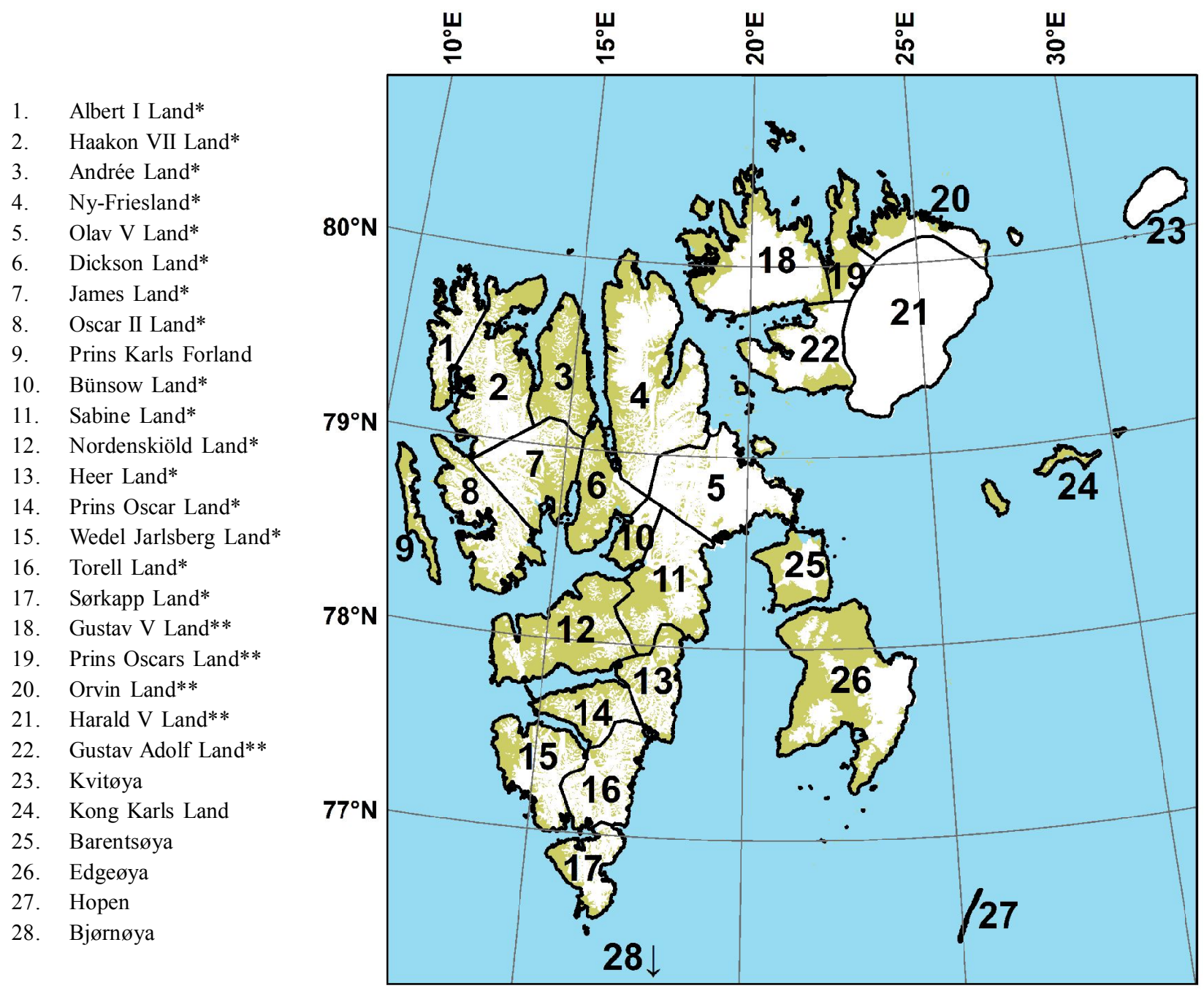

Fig. 1. Geographical districts of Svalbard. 1-8, 10-17 are situated on Spitsbergen (marked with an asterisk, *) and 18-22 on Nordaustlandet (marked with **). Bjørnøya (no. 28) is not shown on map, cf. Fig. 3. Based on Norwegian Polar Institute (2003) with additions from https://stadnamn.npolar.no/sak?sort=-\% $40 \mathrm{id}$.

The first part of Svalbard sighted was the westernmost island which was named "Spitsbergen" not knowing that it was an archipelago. The archipelago was officially named Svalbard around the 1920's, and Spitsbergen restricted to the largest island. However, the name Spitsbergen has been used frequently for the whole archipelago also later and is to some degree still so.

The archipelago consists of several islands with Spitsbergen and Nordaustlandet being the largest. Geographically the archipelago is divided into 28 districts, 6 consists of islands or smaller archipelagos, 5 are situated on Nordaustlandet and 16 on Spitsbergen (Norwegian Polar Institute 2003; see Fig. 1).

About $60 \%$ of Svalbard is glaciated, $30 \%$ barren rocks and $10 \%$ vegetated. On a global scale the ice-free land is classified as "Arctic Desert" (Ecoregions of the World; Olson et al., 2001). However, on a more local scale the flora and vegetation vary within the archipelago. Elvebakk (1999) divided Svalbard into three subzones, Arctic Polar Desert, Northern Arctic Tundra and Middle
Arctic Tundra. Along the western coast and fjords of Spitsbergen the flora is quite diverse given the high latitudes of the islands. A main explanation for this is the proximity to the Gulf Stream (Rønning, 1996).

The Arctic land areas have over the last 2-3 decades experienced more warming than any other region on earth (ACIA, 2005). Alarmingly, novel projections focussing on the Svalbard region indicate a future warming rate up to year 2100 three times stronger than observed during the latest 100 years (Førland, 2011). Today the glaciers are retreating fast, and new land becomes available to colonize (Nuth et al., 2013). This newly available land areas seem to be quickly colonized by liverworts and other plants which was noted during our recent expedition to SW Spitsbergen.

Checklists are powerful and important tools that can integrate the almost overwhelmingly scattered information concerning taxonomy, systematics, nomenclature, distribution, and even frequency (Söderström et al., 2008). We here present a checklist for liverworts of Sval- 


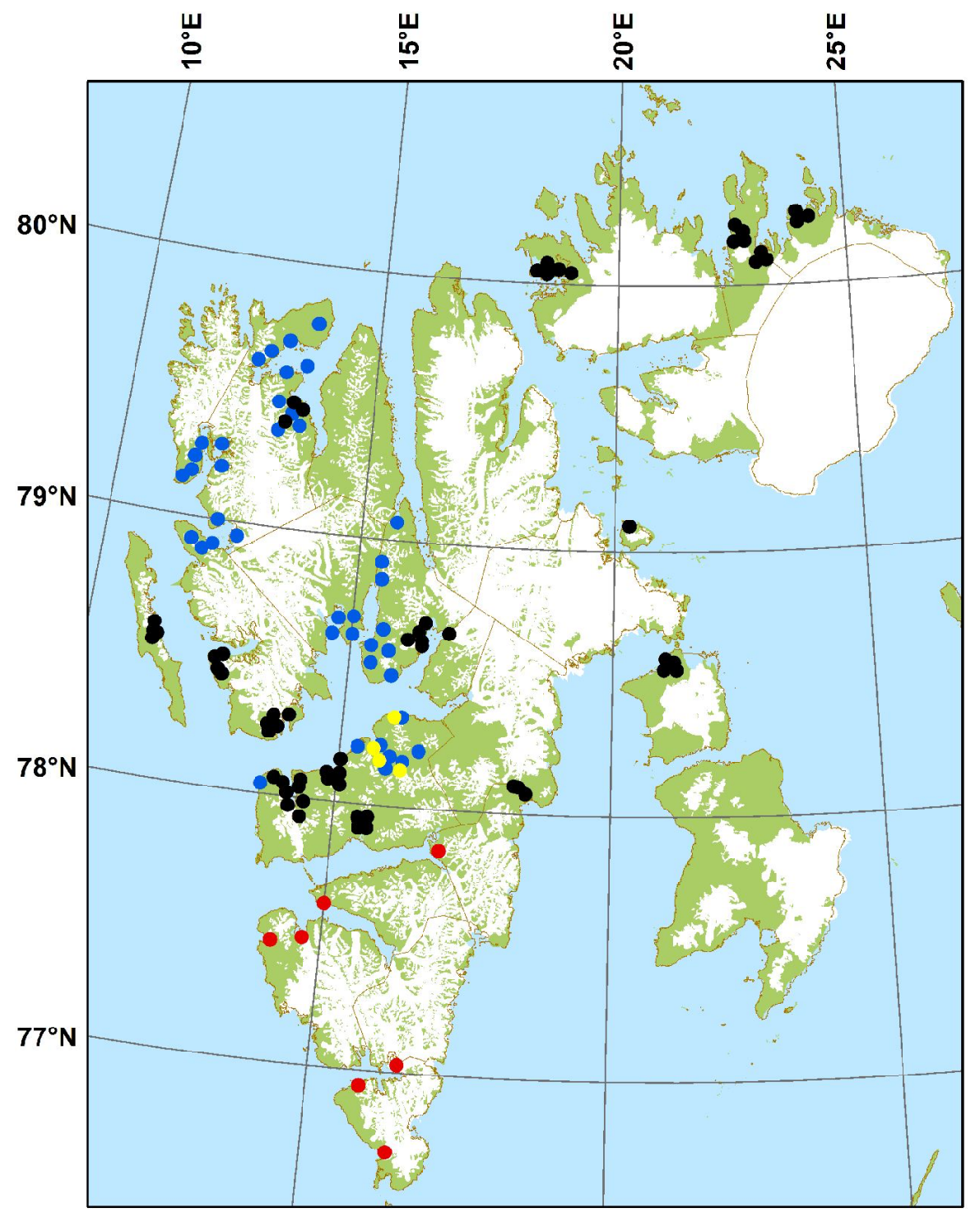

Fig. 2. Main collection points for Frisvoll (blue dots), Konstantinova and co-workers (black dots), NBS excursion (yellow dots) and the NorwegianRussian 2018 expedition (red dots).

bard derived from all published references that we are aware of as well as some new regional records for Svalbard.

\section{RECENT EXPLORATIONS}

The earliest record of bryophytes from the archipelago was by Phips (1777) who reported five mosses and two liverworts. The bryological exploration from the earliest time to 1875 is described by Frisvoll \& Elvebakk (1996), who also made good comments on later sources.

Recent bryological exploration is scattered, but three main projects resulting in the increase of the knowledge of liverwort distribution on the islands should be mentioned.

Arne Frisvoll made three main visits to the archipelago between 1973 and 1977. The "Man and the Biosphere Programme" (MAB) focusing on Svalbard reindeer ecology included mapping of flora and vegetation making it possible for Frisvoll to study the bryophytes. In 1973 he visited the area around Nordfjord (James Land and Dickson Land), in 1974 he studied the NW area of Spitsber- gen (Nordenskiöld Land, Oscar II Land, Haakon VII Land and Albert I Land) and in 1977 Nordenskiöld Land (mainly Adventdalen near Longyearbyen; Fig. 2). The collections of Frisvoll are deposited in TRH and all data registered, but there are many specimens which are yet identified only to genus (e.g., Lophozia s.lat., Cephalozia, Cephaloziella and Scapania). The majority of his collections were identified by him and published. These results, along with a critical overview of early publications, are given by him in the incredibly valuable Cata$\log$ of Bryophytes of Svalbard in Frisvoll \& Elvebakk (1996) which for the past 25 years has been the main guide to the flora of the liverworts of Svalbard.

Since 2004, within the framework of the Russian Interdepartmental Program of Scientific Research of the Nature of Svalbard, the Polar-Alpine Botanical GardenInstitute has studied liverworts of the archipelago under the leadership of N.A. Konstantinova. A brief description of the Russian expeditions that explored the flora and vegetation of the Svalbard archipelago is given in chapter 3 


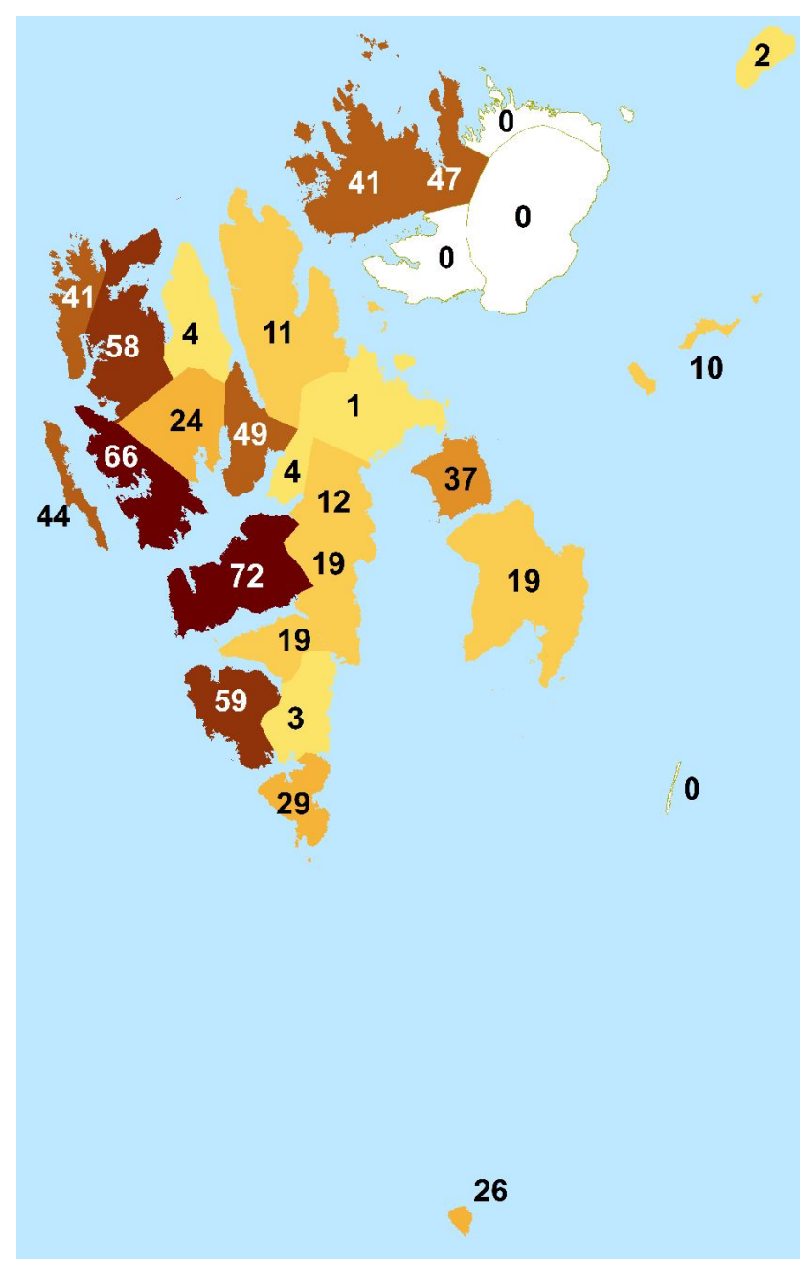

of the book "Science on Svalbard. The history of Russian research" (Konstantinova et al., 2009). Along with an outline of vegetation, this chapter provides the detailed maps of the regions with collection sites that were studied from 2004 to 2008 but provides only the most general information about the most common and some rare liverworts species as well as the general ideas on diversity of liverworts of Svalbard. From 2008 until 2017, the Russian group annually visited additional localities. In total, liverworts have been collected in 15 main areas (Fig. 2, Tab. 1) including five in Nordaustlandet. Three of the collecting areas were visited several times (Colesbukta, vicinity of Pyramiden and Barentsburg towns). Basically, the samples were collected during 5-8 days within a radius of 5-7 km, rarely $10 \mathrm{~km}$ from the location base.

Only a part of the specimens has been identified but annotated lists of species of vicinity of Barentsburg, Nordenskiöld Land (Konstantinova \& Savchenko, 2008b), north coast of Murchisonfjorden, Gustav V Land on Nordaustlandet (Konstantinova \& Savchenko, 2012), the Barents Island (Konstantinova \& Savchenko, 2018) and Prins Karls Forland (Konstantinova \& Savchenko, 2020a) have been published. Findings of new and rare species for some districts of Svalbard have been published (Konstantinova \& Savchenko, 2008a; Konstantinova et al., 2014), particularly from the vicinity of the Pyramiden,
Fig. 3. Number of species known from each district. The darker colour, the more species known.

Dickson Land (Konstantinova \& Savchenko, 2008c), Bockfjorden, Haakon VII Land (Konstantinova \& Savchenko, 2006), and Nordaustlandet (Konstantinova $\&$ Savchenko, 2015) including some that recently have been described from the other parts of Arctic, e.g., Lophozia fuscovirens (Ellis et al., 2019c).

In 2013 the Nordic Bryological Society had a weeklong excursion attended by 15 bryologists visiting several places in Adventdalen and Diabasodden (Fig. 2). After this a photographic flora of some bryophytes in Adventdalen was published (Prestø et al., 2014).

As part of a joint Norwegian-Russian collaboration, a group of six bryologists participated in a field trip in 2018 visiting eight localities on the SW coast of Spitsbergen from Sørkapp Land in the south to Isfjorden in the north. The expedition was led by L. Söderström, Trondheim, and N.A. Konstantinova, Kirovsk, and included Tommy Prestø, Trondheim, Matt von Konrat, Chicago, Anatoliy Savchenko, Kirovsk, and Mikhail Kozhin, Moscow. In addition, the lichenologist Lyudmila Konoreva, Kirovsk, participated. The base for this expedition was a ship with which we started to travel overnight to the first collecting point in Sørkapp Land. The expedition worked its way north along the coast and fjords with 1-2 stops each day (Fig. 2).

The first locality visited ( $15^{\text {th }}$ Aug.) was Stormbukta ( $\left.76^{\circ} 43^{\prime} \mathrm{N}, 16^{\circ} 19^{\prime} \mathrm{E}\right)$ on Sørkapp Land. The area was dry, and the bryophyte vegetation scattered and richest in the wettest parts. In the depressions along the beds of temporary streams, some bryophytes common in Svalbard were found. In addition to species widespread in Svalbard some rare species were also found. However, in total the bryophyte flora of this area is poor.

Next locality to visit ( $16^{\text {th }}$ Aug.) was below Mezenryggen in Torrell Land $\left(77^{\circ} 03^{\prime} \mathrm{N}, 16^{\circ} 30^{\prime} \mathrm{E}\right)$. This is a newly ice-free area between two glaciers, Storbreen and Hornbreen, with only sparse vegetation. The depressions were wet and mostly clayey, and the bryophytes were scattered. However, the bryophyte flora was rather rich, considering the short time it had been ice-free.

In the afternoon, a short stop was made below Krykkjestupet on the N side of Sørkapp Land ( $\left.76^{\circ} 58^{\prime} \mathrm{N}, 16^{\circ} 00^{\prime} \mathrm{E}\right)$ where a couple of interesting species were found.

On the $17^{\text {th }}$ Aug. the $\mathrm{N}$ side of Dunderbukta below Dunderfjellet on Wedel Jarlsberg Land (77²9'N, $\left.14^{\circ} 01^{\prime} \mathrm{E}\right)$ was visited. Of all studied sites on the expedition this was the richest both in abundance and diversity of bryophytes. Common and sometimes abundant were species that are widespread in Svalbard, but also several rare species were found.

On the $18^{\text {th }}$ Aug. Ottoneset on Heer Land $\left(77^{\circ} 50^{\prime} \mathrm{N}\right.$, $\left.16^{\circ} 49^{\prime} \mathrm{E}\right)$ was visited. The bryophyte flora is relatively poor in sense of species diversity but some species widespread in Svalbard were rather abundant here. 
Table 1. Main investigation sites for Russian expeditions 2004-2016. Coordinates are for the base camp at each locality.

\author{
Visiting period \\ 23 July - 1 August 2004 \\ $21-24$ July 2005 \\ $27-29$ July 2005 \\ 27 July - 1 August 2006 \\ $3-7$ August 2006 \\ $2-10$ August 2007
}

August $13-20,2007$

$18-21$ July 2008

25 July -2 August 2008

29 July - 2 August 2009

$6-10$ August 2009

$23-29$ July 2010

$2-5$ August 2010

$23-30$ July 2011

$3-6$ August 2011

$15-18$ July 2012

$27-31$ July 2012

$22-31$ July 2014

2 August 2015

5 - 9 August 2015

13 August 2015

2 - 9 July 2016
Main investigation areas with coordinates of base camp

Spitsbergen, Nordenskiöld Land: Grønfjord Area $78^{\circ} 03^{\prime} 48^{\prime \prime} \mathrm{N}, 14^{\circ} 15^{\prime} 07^{\prime \prime} \mathrm{E}$

Spitsbergen, Nordenskiöld Land: Kapp Starostin $78^{\circ} 04^{\prime} 48^{\prime \prime} \mathrm{N}, 13^{\circ} 44^{\prime} 44^{\prime \prime E}$

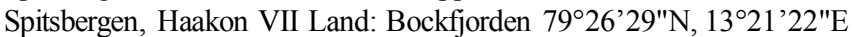

Spitsbergen, Nordenskiöld Land: Reindalen. $77^{\circ} 59^{\prime} 46^{\prime \prime} \mathrm{N}, 15^{\circ} 51^{\prime} 27^{\prime \prime} \mathrm{E}$

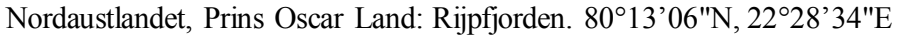

Spitsbergen, Nordenskiöld Land: Barentsburg- Gunnarvarden, Storheia.

$78^{\circ} 05^{\prime} 02^{\prime \prime} \mathrm{N}, 14^{\circ} 13^{\prime} 29^{\prime \prime} \mathrm{E}$

Nordaustlandet, Gustav V Land: Murchisonfjorden, Nordvika.

$80^{\circ} 02^{\prime} 03^{\prime \prime} \mathrm{N}, 18^{\circ} 49^{\prime} 07^{\prime \prime} \mathrm{E}$

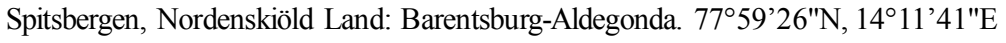

Spitsbergen, Dickson Land: Billefjorden, Pyramiden. 78 $39^{\prime} 20^{\prime \prime} \mathrm{N}, 16^{\circ} 21^{\prime} 08^{\prime \prime} \mathrm{E}$

Spitsbergen, Nordenskiöld Land: Rusanovodden, Colesbukta. 7807’ $52^{\prime \prime} \mathrm{N}, 14^{\circ} 59^{\prime} 16^{\prime \prime} \mathrm{E}$

Spitsbergen, Oscar II Land: Trygghamna. 78 $13^{\prime} 49^{\prime \prime} \mathrm{N}, 13^{\circ} 48^{\prime} 53^{\prime \prime} \mathrm{E}$

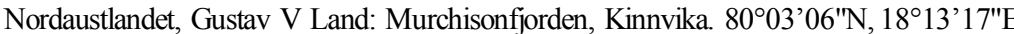

Spitsbergen, Sabine Land: Agardhbukta. $78^{\circ} 04^{\prime} 51^{\prime \prime N}, 18^{\circ} 30^{\prime} 52^{\prime \prime} \mathrm{E}$

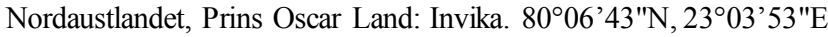

Barentsøya, Frankenhalvøya: Heimland. $78^{\circ} 35^{\prime} 09^{\prime \prime} \mathrm{N}, 21^{\circ} 06^{\prime} 45^{\prime \prime} \mathrm{E}$

Spitsbergen, Oscar II Land: Ymerbukta. $78^{\circ} 14^{\prime} 41^{\prime \prime} \mathrm{N}, 13^{\circ} 55^{\prime} 50^{\prime \prime} \mathrm{E}$

Nordaustlandet, Orvin Land: Polarkluben. $80^{\circ} 16^{\prime} 01^{\prime \prime N}, 23^{\circ} 57^{\prime} 50^{\prime \prime E}$

Spitsbergen, Oscar II Land: St. Jonsfjorden. $78^{\circ} 28^{\prime} 18^{\prime \prime N}, 12^{\circ} 23^{\prime} 33^{\prime \prime E}$

Spitsbergen, Nordenskiöld Land: Barentsburg, Grumantbyen. 78¹0’30"N, 1506’38"E

Spitsbergen, Nordenskiöld Land: Rusanovodden, Colesbukta. 7807’52"N, 1459'16"E

Spitsbergen, Nordenskiöld Land: Longyearbyen, Platåberget. 78¹2’59"N, 15³6’47"E

Prins Karls Forland, Selvågen. $78^{\circ} 33^{\prime} 02^{\prime \prime N}, 11^{\circ} 15^{\prime} 28^{\prime \prime} \mathrm{E}$
Number of Number of colstudied sites lected specimens

$48 \quad 180$

$16 \quad 90$

$10 \quad 80$

$64 \quad 155$

$57 \quad 153$

14

140

29

120

18

222

183

197

160

110

158

56

127

154

96

30

83

16

92
On the $19^{\text {th }}$ Aug. a visit to the $\mathrm{N}$ side of Nathorst Land in Van Mijenfjorden was planned, but the wind made landing impossible. Instead, the expedition visited Svarthamaren on the E side of Recherchefjorden on Wedel Jarlsberg Land $\left(77^{\circ} 32^{\prime} \mathrm{N}, 14^{\circ} 15^{\prime} \mathrm{E}\right)$. The bryophyte flora of this site is rather typical for some sites in inner fjord areas of Svalbard. Several species that are widespread in Svalbard are rather abundant but also some species that were regarded as rare in Svalbard occur sporadically here.

The wind made landing difficult also next day, but the expedition was able to visit Strandvollsletta below Bravaisberget on the S side of Nathorst Land $\left(77^{\circ} 37^{\prime} \mathrm{N}, 14^{\circ} 55^{\prime} \mathrm{E}\right)$. This is a rich site where we found many species, both common and widespread ones and more rare species.

A fair part, but not all, of the collected specimens from this expedition is identified. However, among the identified, there are many new species records for the visited areas, increasing the knowledge of their distribution on Svalbard.

In spite of all those expeditions, only one species, $\mathrm{LO}$ phozia svalbardensis, has been described from Svalbard during the last 20 years (Konstantinova et al., 2020a).

\section{METHODS}

In this report, we report all published references to liverworts on Svalbard that we are aware of has been included. This includes both floristic reports, reports of voucher specimens from taxonomic reports, list of species from phytosociological and other ecological reports, as well as occasional citations in other sources we have come across.
Many of those references only mention Svalbard (or Spitsbergen) in a general outline of the occurrence of a species. As there are several errors in such reports, we include them in the list but comment on the ones we think are erroneous or when it is unclear to us what primary data such a report is based on. When only the term "Spitsbergen" is used we refer it to Svalbard, unless further information (e.g. a reference to place name on Spitsbergen that can not be placed for certain in any district) is available.

We also cite many specimens from districts of Svalbard where they have not been published earlier, some new for a district, some for one of the major islands and one new genus for Svalbard.

Arne Frisvoll (in Frisvoll \& Elvebakk, 1996) discussed many doubtful reports and rejected many taxa from the archipelago. Most of them were old names where the concept has changed, or names of very unlikely taxa occurring in list of species from various districts. Some of them have later proven to occur on Svalbard, but we also doubt or exclude some other more recently reported taxa.

Some old names, mainly of infraspecific taxonomic rank, have never been dealt with since their description $100-150$ years ago. We have not been able to find types for some of them, and we thus just mention the names without placing them anywhere. Two of the names are typified here and their reports moved to the taxon they are synonymized with.

The nomenclature follows Hodgetts et al. (2020) except for Blepharostoma brevirete and Protochilopsis grandiretis which have been published recently. 


\section{NEW REPORTS}

We here list specimens from districts where they have not been reported earlier in the literature. We mostly report only one specimen from each district although we are often aware of several specimens. Some of the specimens are for the first time reported from Svalbard here, others are new for one of the larger islands while the majority of the reports are new to a district. Specimens are mainly deposited in TRH (Frisvoll's specimens) or KPABG (Konstantinova's specimens), in addition to a few in TROM (extracted from GBIF data).

Aneura pinguis (L.) Dumort.

- Spitsbergen, James Land: Ekmanfjorden. Coraholmen ( $78^{\circ} 40^{\prime}$ N, $14^{\circ} 42^{\prime}$ E) , 18.VI.1973 Frisvoll, det. N.A. Konstantinova 2016 (TRH B74281/a). New to James Land.

- Spitsbergen, Nathorst Land: Bellsund. Van Keulenfjorden. Strandvollsletta $\left(77^{\circ} 37^{\prime} 22^{\prime \prime} \mathrm{N}, 14^{\circ} 54^{\prime} 47^{\prime \prime} \mathrm{E}\right)$, hollow with huge rocks and small stream, at base of slope. 19.VIII.2018 Konstantinova \& Savchenko K59-18 (KPABG 123698). New to Nathorst Land.

Anthelia juratzkana (Limpr.) Trevis.

- Spitsbergen, Albert I Land: Lilliehöökfjorden, Signehamna, ved

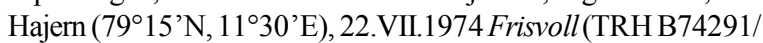
1). New to Albert I Land.

- Spitsbergen, Heer Land: Ottoneset, 7 m a.s.1. (77'50'14"N, $16^{\circ} 49^{\prime} 19^{\prime \prime E}$ ), Arctic heathland. Thin peaty soil in stony slope, 18.VIII.2018 Söderström 2018/011. New to Heer Land.

- Spitsbergen, James Land: Blomesletta (78 $\left.37^{\circ} 41,14^{\circ} 51^{\prime} \mathrm{E}\right)$, volcanic rock on the plain, 26.VII.1973 Frisvoll, det. Prestø 2004 (TRH B74294/1). New to James Land.

- Spitsbergen, Nathorst Land: Strandvollsletta, $3 \mathrm{~m}$ a.s.1. $\left(77^{\circ} 37^{\prime} 20^{\prime \prime} \mathrm{N}, 14^{\circ} 54^{\prime} 22^{\prime \prime} \mathrm{E}\right)$, On moist peaty heathland, 20.VIII.2018 Söderström 2018/022. New to Nathorst Land.

Arnellia fennica (Gottsche) Lindb.

- Spitsbergen, Sabine Land: Moskusdalen mot Sassendalen, på sørsiden av Brattlihøgda (78¹7’N, 17³4'E), , VII.1990 Bryhn Jacobsen, det. Frisvoll (TRH B74064/1). New to Sabine Land. Mentioned to occur in Moskusdalen by Frisvoll \& Elvebakk (1996) but without citing any specimen.

Barbilophozia barbata (Schmidel ex Schreb.) Loeske

- Spitsbergen, Albert I Land: Lilliehöökfjorden. Ved Hajern (Signehamna) $\left(79^{\circ} 15^{\prime} \mathrm{N}, 11^{\circ} 30^{\prime} \mathrm{E}^{\prime}\right), 22$. VII.1974 Frisvoll 74085 (KPABG 122643). New to Albert I Land.

Barbilophozia hatcheri (A.Evans) Loeske

— Nordaustlandet, Prins Oscars Land: Innvika Bay. Ringgåsdalen, eastern shore of the Ringgåsvatnet, right bank of Ringgåsdalen River $\left(80^{\circ} 06^{\prime} 19^{\prime \prime} \mathrm{N}, 23^{\circ} 01^{\prime} 50^{\prime \prime} \mathrm{E}\right)$, the bottom of north facing slope. 25.VII.2011 Konstantinova K309-6-11 (KPABG 115412). New to Prins Oscars Land.

- Spitsbergen, Dickson Land: Sauriedalen. Toppen av Ibsenfjella V (78³3’N, 15³4'E), 31.VII.1973 Frisvoll (TRH B74076/ 1). New to Dickson Land.

- Spitsbergen, Haakon VII Land: Biscayahuken mot Yermaktangen (7950'N, $\left.12^{\circ} 23^{\prime} \mathrm{E}\right), 18$.VIII.1958 Rønning, det. A.A. Frisvoll 1979 (TRH B74502/1). New to Haakon VII Land.

Barbilophozia sudetica (Nees ex Huebener) L.Söderstr., De Roo et Hedd.

- Spitsbergen, Dickson Land: Kap Wijk, S of Oxaashytta $\left(78^{\circ} 33^{\prime} 59^{\prime \prime} \mathrm{N}, 15^{\circ} 14^{\prime} 40^{\prime \prime} \mathrm{E}\right)$, Low arctic tundra. On soil in wet heathland, 22 m a.s.1., 29.VIII.2004 Söderström 2004/345. New to Dickson Land.
Blepharostoma brevirete (Bryhn et Kaal.) Vilnet et Bakalin

- Spitsbergen, Nathorst Land: Bellsund. Van Keulenfjorden. Strandvollsletta $\left(77^{\circ} 37^{\prime} 22^{\prime \prime} \mathrm{N}, 23^{\circ} 01^{\prime} 50^{\prime \prime} \mathrm{E}\right)$, steep slope, south exposure covered by mosses with scattered Oxyria digyna, Polygonum viviparum, Saxifraga oppositifolia, between gravel on loamy soil. 19.VIII.2018 Konstantinova \& Savchenko K503a-18 (KPABG 123677). New to Nathorst Land.

- Spitsbergen, Ny-Friesland: Outer part of Ringhorndalen, northern entrance to the valley, north side of the westernmost lake $\left(79^{\circ} 20^{\prime} \mathrm{N}, 16^{\circ} 01^{\prime} \mathrm{E}\right)$. 4.VIII.2017 Bronken Eidesen, det. Hassel (TRH B39458/4). New to Ny-Friesland. However, the report of Blepharostoma trichophyllum by Wulf (1902) probably belongs here.

- Spitsbergen, Sørkapp Land: Stormbukta $\left(76^{\circ} 42^{\prime} 45^{\prime \prime} \mathrm{N}, 016^{\circ} 17^{\prime} 46^{\prime \prime} \mathrm{E}\right)$, in crevices at the base of the outliers. 14.VIII.2018 Konstantinova \& Savchenko K2-1b-18 (KPABG 123540). New to Sørkapp Land.

Cephalozia ambigua C.Massal.

- Nordaustlandet, Prins Oscars Land: east coast of Rijp Fjorden, north faced cliffs of Bluffvarden Mount on southern bank of Heieren Lake $\left(80^{\circ} 12^{\prime} 39^{\prime \prime} \mathrm{N}, 2^{\circ} 28^{\prime} 31^{\prime \prime} \mathrm{E}\right)$, slope mount, on muddy rocks in mossy bed of temperate stream. 4.VII.2006 Konstantinova \& Savchenko K78-1f-15 (KPABG 122999). New to Prins Oscars Land.

- Spitsbergen, Haakon VII Land: Reinsdyrflya, Worsleyhamna (7941’N, 1326'E), lichen heath, 5.VIII.1981 Dahle. (TRH B732473/16). New to Haakon VII Land.

Cephaloziella integerrima (Lindb.) Warnst.

- Spitsbergen, Sørkapp Land: Stormbukta $\left(76^{\circ} 42^{\prime} 53^{\prime \prime N}\right.$, $16^{\circ} 19^{\prime} 01^{\prime \prime E}$ ), hollow, the bed of a temporary watercourse, at base of rock. 14.VIII.2018 Konstantinova \& Savchenko K5-1a18 (KPABG 123543)]. New to Sørkapp Land.

- Spitsbergen, Wedel Jarlsberg Land: Dunderbukta, Dunderdalen, northern part $\left(77^{\circ} 28^{\prime} 47^{\prime \prime} \mathrm{N}, 14^{\circ} 02^{\prime} 10^{\prime \prime} \mathrm{E}\right)$, on slope, on side of earth hummock. 16.VIII.2018 Konstantinova \& Savchenko K242-18 (KPABG 123728). New to Wedel Jarlsberg Land.

Cephaloziella uncinata R.M.Schust.

- Spitsbergen, Dickson Land: Dicksonfjorden. Idodalen, Sskråningen av Heimenfjellet (73³4'N, 15²6'E), 29.VII.1973 Frisvoll, det. Prestø 2004 (TRH B732311/1). New to Dickson Land.

- Spitsbergen, Haakon VII Land: Kongsfjorden. Ossian Sarsfjellet, Alkekongedal. 20.VII.1974 Frisvoll, det. Hassel 2021 (TRH 67010/2). New to Haakon VII Land.

Cephaloziella varians (Gottsche) Steph.

- Spitsbergen, Heer Land: Van Mijenfjorden. Rindersbukta. Ottoneset $\left(76^{\circ} 57^{\prime} 56^{\prime \prime} \mathrm{N}, 16^{\circ} 00^{\prime} 04^{\prime \prime} \mathrm{E}\right)$, on solifluction mantle, on loamy soil. 17.VIII.2018 Konstantinova \& Savchenko K37-1a18 (KPABG 123646). New to Heer Land.

- James Land: Dicksonfjorden, in the valley between Tolmodryggen and Trollfuglfjella ( $\left.78^{\circ} 40^{\prime} \mathrm{N}, 14^{\circ} 58^{\prime} \mathrm{E}\right)$. 27.VII.1973 Frisvoll, det. N.A. Konstantinova 2016 (TRH B73799/2)]. New to James Land.

- Spitsbergen, Nathorst Land: Bellsund. Van Keulenfjorden. Strandvollsletta $\left(77^{\circ} 37^{\prime} 23^{\prime \prime} \mathrm{N}, 14^{\circ} 54^{\prime} 16^{\prime \prime} \mathrm{E}\right)$, hollow with huge rocks, bank of small stream, on rock. 19.VIII.2018 Konstantinova \& Savchenko K56-18 (KPABG 123690). New to Nathorst Land.

- Spitsbergen, Sørkapp Land: Hornsund Bay, Rasstupet bird cliffs Krykkjestupet $\left(76^{\circ} 57^{\prime} 56^{\prime \prime} \mathrm{N}, 16^{\circ} 00^{\prime} 04^{\prime \prime} \mathrm{E}\right)$, at base of large rocky outcrops, (weakly altered carbonate rocks, seeping cliffs), steep mossy slope dominated with Cochlearia arctica, Oxyria digy- 
$n a$ and mosses, on mosses. 15.VIII.2018 Konstantinova \& Savchenko K19-1-2-18 (KPABG 123603). New to Sørkapp Land.

Clevea hyalina (Sommerf.) Lindb.

- Spitsbergen, Nordenskiöld Land: Adventdalen. Dryas-polygon i Foxdalen V, ved elv fra Foxfonna fremst i dalen $\left(78^{\circ} 09^{\prime} \mathrm{N}\right.$, 16²'’). 3.VIII.1977 Frisvoll 73831 (KPABG 122666). New to Nordenskiöld Land.

- Spitsbergen, Wedel Jarlsberg Land: Dunderbukta, Dunderdalen northern part $\left(77^{\circ} 28^{\prime} 45^{\prime \prime} \mathrm{N}, 1^{\circ} 01^{\prime} 53^{\prime \prime} \mathrm{E}\right)$, Ptilidium dominated tundra, on loamy soil on nonsorted circle. 16.VIII.2018 Konstantinova \& Savchenko K20-3a-18 (KPABG 123613). New to Wedel Jarlsberg Land.

Diplophyllum taxifolium (Wahlenb.) Dumort.

- Nordaustlandet, Prins Oscars Land: east coast of Rijp Fjorden, north faced cliffs of Bluffvarden Mount on southern bank of Heieren Lake $\left(80^{\circ} 12^{\prime} 39^{\prime \prime} \mathrm{N}, 22^{\circ} 28^{\prime} 31^{\prime \prime} \mathrm{E}\right)$, slope mount, on muddy rocks in mossy bed of temperate stream. 4.VII.2006 Konstantinova \& Savchenko K78-1b-15 (KPABG 122996). New to Nordaustlandet.

Fuscocephaloziopsis albescens (Hook.) Váňa et L.Söderstr.

- Nordaustlandet, Prins Oscars Land: East coast of Rijp Fjorden, north faced cliffs of Bluffvarden Mount on southern bank of Heieren Lake $\left(80^{\circ} 12^{\prime} 39^{\prime \prime} \mathrm{N}, 2^{\circ} 28^{\prime} 31^{\prime \prime} \mathrm{E}\right)$, slope mount, on muddy rocks in mossy bed of temperate stream. 4.VII.2006 Konstantinova \& Savchenko K78-1f-15 (KPABG 122999). New to Nordaustlandet.

- Spitsbergen, Dickson Land: Billefjorden SV. Rotundafjellet (78²9’N, 1550'E), 19.VIII.1973 Frisvoll, det. N.A. Konstantinova 2016 (TRH B73819/1). New to Dickson Land.

Fuscocephaloziopsis pleniceps (Austin) Váňa et L.Söderstr.

- Nordaustlandet, Prins Oscars Land: South of Blyfjorden, valley of an unnamed large river that flows into Ripfjorden (80¹1’33"N, 22³0’47"E). 6.VIII.2006 Konstantinova Ę1151-06 (KPABG 111814). New to Nordaustlandet.

— Spitsbergen, James Land: Blomesletta (78³7’41, 1451’E), volcanic rock on the plain, solifluction ground in boulder field. 28.VII.1973 Frisvoll (TRH B73891/1). New to James Land but mentioned in Frisvoll \& Elvebakk (1996) as unpublished without any specimen detail.

Gymnocolea inflata (Huds.) Dumort.

- Nordaustlandet, Prins Oscars Land: west coats of Invika Bay, north-east end of Ringgåsdalen, on soil in bed of a weak-flowing stream, submerged. 28.VII.2011 Konstantinova \& Savchenko K373-5-11 (KPABG 124049. Mentioned as new for Nordaustlandet in Konstantinova \& Savchenko (2015b) without citing any specimen.

Gymnomitrion concinnatum (Lightf.) Corda

— Spitsbergen, Dickson Land: Nordfjorden. Kongressfjellet N, 20.VII.1973. Frisvoll (TRH B73631/1). New to Dickson Land although mentioned in Belkina et al. (2015) without citing any specimen.

Gymnomitrion corallioides Nees

- Spitsbergen, Dickson Land: Sauriedalen. Toppen av Ibsenfjella V (78³3'N, 15³4'E), 31.VII.1973 Frisvoll (TRH B74012/ 1). New to Dickson Land.

- Ny-Friesland: Mosselhalvøya (79 $\left.56^{\prime} \mathrm{N}, 16^{\circ} 18^{\prime} \mathrm{E}\right)$, under the edge of a boulder in a boulder field. 22.VII.2008 Hagen, det. Prestø 2008 (TRH B38443/2). New to Ny-Friesland.

- Oscar II Land: Kongsfjorden. Ny-Ålesund, Zeppelinerhamna (78 55’ N, 11 59'E), 27.VI.1974 Frisvoll (TRHB74015/1). New to Oscar II Land.
Jungermannia polaris Lindb.

— Nordaustlandet, Prins Oscars Land: Innvika Bay, north-west coast of Depotlaguna, left bank of Ringgåsdalen River $\left(80^{\circ} 06^{\prime} 48^{\prime \prime} \mathrm{N}, 23^{\circ} 03^{\prime} 48^{\prime \prime} \mathrm{E}\right)$, landslide on slope to the river. 30.VII.2011 Konstantinova K343-2c-11 (KPABG 115466)]. New to Prins Oscars Land.

- Spitsbergen, Heer Land: Van Mijenfjorden. Rindersbukta. Ottoneset, at base of slope $\left(77^{\circ} 50^{\prime} 13^{\prime \prime} \mathrm{N}, 16^{\circ} 49^{\prime} 46^{\prime \prime} \mathrm{E}\right)$, grassmoss community on waterlogged soil. 17.VIII.2018 Konstantinova \& Savchenko K38-1-18 (KPABG 123705). New to Heer Land.

- Spitsbergen, James Land: Blomesletta, på østsida nær hytte (78³7’N, 1458’E), 4.VII.1973 Frisvoll (TRH B732315/2). New to James Land.

- Spitsbergen, Nathorst Land: Bellsund. Van Keulenfjorden. Strandvollsletta $\left(77^{\circ} 37^{\prime} 22^{\prime \prime} \mathrm{N}, 1^{\circ} 54^{\prime} 47^{\prime \prime E}\right)$, hollow with huge rocks and small stream, rock field at base of slope, on rock. 19.VIII.2018 Konstantinova \& A.N.Savchenko K58-1e-18 (KPABG 123696). New to Nathorst Land.

- Spitsbergen, Sørkapp Land: Stormbukta (76²4'09"N, $\left.16^{\circ} 18^{\prime} 44^{\prime \prime} \mathrm{E}\right)$, at the base of the slope of the moraine terrace, moss tundra, on humus soil on side a hummock 4-5 cm high, among mosses. 14.VIII.2018 Konstantinova \& Savchenko K92-18 (KPABG 123553). New to Sørkapp Land.

- Spitsbergen, Wedel Jarlsberg Land: Recherchefjorden. Lagerneset ( $\left.77^{\circ} 31^{\prime} 08^{\prime \prime} \mathrm{N}, 14^{\circ} 48^{\prime} 09^{\prime \prime} \mathrm{E}\right)$, gentle slope with numerous rivulets and moss mats, on side of hummock. 18.VIII.2018 Konstantinova \& Savchenko K49-4b-18(KPABG 123741). New to Wedel Jarlsberg Land.

Jungermannia pumila With.

- Spitsbergen, Dickson Land: Billefjorden. Pyramiden, southeast coast of Mimerbukta, at the bottom of Yggdrasilkampen Mtn, delta of Mimerselva ( $\left.78^{\circ} 38^{\prime} 18^{\prime \prime} \mathrm{N}, 16^{\circ} 20^{\prime} 52^{\prime \prime} \mathrm{E}\right)$, steep north facing slope, in upper part of bank of deep cutting brook, on soil near rock. 28.VII.2008 Konstantinova K54-1-08 (KPABG 118807). New to Dickson Land.

- Spitsbergen, Nathorst Land: Bellsund. Van Keulenfjorden. Strandvollsletta $\left(77^{\circ} 37^{\prime} 22^{\prime \prime} \mathrm{N}, 1^{\circ} 54^{\prime} 18^{\prime \prime} \mathrm{E}\right)$, steep slope, south exposure covered by mosses with scattered Oxyria digyna, Polygonum viviparum, Saxifraga oppositifolia, between gravel on loamy soil. 19.VIII.2018 Konstantinova \& Savchenko K501-18 (KPABG 123675). New to Nathorst Land.

- Spitsbergen, Sørkapp Land: Stormbukta $\left(76^{\circ} 42^{\prime} 55^{\prime \prime N}\right.$, $\left.16^{\circ} 18^{\prime} 42^{\prime \prime E}\right)$, under rock on the slope of the northern exposition. 14.VIII.2018 Konstantinova \& Savchenko K4-2-18 (KPABG 123542). New to Sørkapp Land.

- Spitsbergen, Wedel Jarlsberg Land: Dunderbukta, Dunderdalen, northern part $\left(77^{\circ} 28^{\prime} 47^{\prime \prime} \mathrm{N}, 14^{\circ} 02^{\prime} 20^{\prime \prime} \mathrm{E}\right)$, on side of moss covered rock, on peat soil. 16.VIII.2018 Konstantinova \&.Savchenko K26-2b-18 (KPABG 123625)]. New to Wedel Jarlsberg Land. Lophozia fuscovirens Bakalin et Vilnet

- Spitsbergen, Heer Land: Van Mijenfjorden. Rindersbukta. Ottoneset cabin, valley between two hills $\left(77^{\circ} 50^{\prime} 13^{\prime \prime} \mathrm{N}\right.$, $\left.16^{\circ} 49^{\prime} 46^{\prime \prime} \mathrm{E}\right)$, north faced slope, nearest part, among small rocks, on soil. 17.VIII.2018 Konstantinova \& Savchenko K39-2-18 (KPABG 123706)]. New to Heer Land.

Lophozia savicziae Schljakov

- Svalbard, Olav V Land, Storfjorden, Ginevrabotnen, Diabastangen, among mosses in tundra (78 $\left.36^{\prime} 27^{\prime \prime} \mathrm{N}, 20^{\circ} 00^{\prime} 34^{\prime \prime E}\right)$. 9.VIII.1899 Birula, det. Bakalin (KPABG 124054). New to Olav $V$ Land. This is the specimen that Bakalin's (2005) unspecified report from Svalbard is based on. 
Lophozia ventricosa (Dicks.) Dumort.

- Nordaustlandet, Prins Oscars Land: Rock outcrops on the northern slope of Bluffvarden (80¹2’39"N, 22²8'31"E). 4.VIII.2006 Konstantinova K79-1-06 (KPABG 111792). New to Nordaustlandet.

Lophozia wenzelii (Nees) Steph.

- Nordaustlandet, Gustav V Land: Kinnvika, Florabukta $\left(80^{\circ} 02^{\prime} 06^{\prime \prime} \mathrm{N}, 18^{\circ} 40^{\prime} 58^{\prime \prime} \mathrm{E}\right)$, at the bottom of cliffs with bird colonies south-west slope of Floraberget. 24.VII.2010 Konstantinova \& Savchenko K16-2-10 (KPABG 114007). New to Nordaustlandet.

- Nordaustlandet, Prins Oscars Land: Innvika Bay, north-west coast of Depotlaguna, left bank of Ringgåsdalen River $\left(80^{\circ} 06^{\prime} 48^{\prime \prime} \mathrm{N}, 23^{\circ} 03^{\prime} 48^{\prime \prime} \mathrm{E}\right)$, landslide on slope to the river. 30.VII.2011 Konstantinova Ę342-11 (KPABG 115457). New to Prins Oscars Land.

Lophoziopsis excisa (Dicks.) Konstant. et Vilnet

- Nordaustlandet, Prins Oscars Land: Innvika Bay, north-west coast of Depotlaguna, left bank of Ringgåsdalen River $\left(80^{\circ} 06^{\prime} 48^{\prime \prime} \mathrm{N}, 23^{\circ} 03^{\prime} 48^{\prime \prime} \mathrm{E}\right)$, landslide on steep slope of sea terrace. 30.VII.2011 Konstantinova Ė341-3ŕ-11 (KPABG 115455). New to Nordaustlandet.

- Spitsbergen, Heer Land: Van Mijenfjorden. Rindersbukta. Ottoneset $\left(77^{\circ} 50^{\prime} 14^{\prime \prime} \mathrm{N}, 16^{\circ} 49^{\prime} 20^{\prime \prime} \mathrm{E}\right)$, solifluction mantle (slope with numerous rocks and loamy soil), on loamy soil. 17.VIII.2018 Konstantinova \& Savchenko K34-1a-18 (KPABG 123635). New to Heer Land.

- Spitsbergen, Sørkapp Land: Hornsund Bay, Rasstupet bird cliffs Krykkjestupet $\left(76^{\circ} 57^{\prime} 47^{\prime \prime} \mathrm{N}, 15^{\circ} 58^{\prime} 49^{\prime \prime} \mathrm{N}\right)$, at base of large rocky outcrops weakly altered carbonate rocks (seeping cliffs), among mosses. 15.VIII.2018 Konstantinova \& Savchenko K14-1a-18 (KPABG 123592). New to Sørkapp Land.

Lophoziopsis jurensis (Meyl. ex Müll.Frib.) Mamontov et Vilnet.

- Nordaustlandet, Prins Oscars Land: Innvika Bay. North-west coast of Depotlaguna, left bank of Ringgåsdalen River, landslide on slope to the river. 30.VII.2011 Konstantinova K3432c-11 (KPABG 115466)]. New to Nordaustlandet.

— Barentsøya: Frankenhalvøya. 6.IX.2011 Savchenko CA-3671b-11 (KPABG 119733). New to Barentsøya.

Lophoziopsis pellucida (R.M.Schust.) Konstant. et Vilnet.

- Spitsbergen, Dickson Land: Billefjorden. Pyramiden, upper part of Mimerdalen, northern slope under glacier Jotunfonna $\left(78^{\circ} 38^{\prime} 03^{\prime \prime} \mathrm{N}, 16^{\circ} 06^{\prime} 46^{\prime \prime} \mathrm{E}\right)$, rocky steep north facing slope on left shore of brook, block field of red Devonian sandstone. In clay soil under rock. 27.VII.2008 Konstantinova K41-2c-08 (KPABG 116544)]. New to Dickson Land.

- Spitsbergen, Ny-Friesland: Ringhorndalen, Dellingstupa $\left(79^{\circ} 20^{\prime} \mathrm{N}, 16^{\circ} 10^{\prime} \mathrm{E}\right)$, in the sanddunes, 13.VIII.2015 Bronken Eidesen, det. Hassel 2017 (TRH B38905/2). New to NyFriesland.

Lophoziopsis polaris (R.M.Schust.) Konstant. et Vilnet.

- Spitsbergen, Dickson Land: Billefjorden. Pyramiden. Mimerdalen, extreme southeastern part of Odinfjellet, Planteryggen Ridge $\left(78^{\circ} 39^{\prime} 48^{\prime \prime} \mathrm{N}, 16^{\circ} 05^{\prime} 52^{\prime \prime} \mathrm{E}\right)$, mossy depression with seepage. In small hollow. 30.VII.2008 Konstantinova K73-3a-08 (KPABG 118863). New to Dickson Land.

- Spitsbergen, James Land: Dicksonfjorden SV. I dalen mellom Tolmodryggen og Trollfuglfjella ( $\left.78^{\circ} 40^{\prime} \mathrm{N}, 14^{\circ} 58^{\prime} \mathrm{E}\right)$, fuglefjell. 27.VII.1973 Frisvoll, det. Konstantinova 2016 (TRH B73838/ 2). New to James Land.

- Spitsbergen, Nathorst Land: Bellsund. Van Keulenfjorden. Strandvollsletta $\left(77^{\circ} 37^{\prime} 22^{\prime \prime} \mathrm{N}, 14^{\circ} 54^{\prime} 47^{\prime \prime E}\right)$, hollow with huge rocks and small stream, rock field at base of slope, on rock. 19.VIII.2018 Konstantinova \& Savchenko K58-1e-18 (KPABG 123696). New to Nathorst Land.

- Spitsbergen, Ny-Friesland: Ringhorndalen, Dellingstupa $\left(79^{\circ} 20^{\prime} \mathrm{N}, 16^{\circ} 10^{\prime} \mathrm{E}\right)$, in the mossy run-off next to the Cassiope heath. 13.VIII.2015 Bronken Eidesen, det. Söderström, 2021 (TRH B38899/2). New to Ny-Friesland.

- Spitsbergen, Sørkapp Land: Hornsund Bay, Rasstupet bird cliffs Krykkjestupet ( $76^{\circ} 57^{\prime} 47^{\prime \prime} \mathrm{N}, 15^{\circ} 58^{\prime} 49^{\prime \prime E}$ ), at base of large rocky outcrops weakly altered carbonate rocks (seeping cliffs), among mosses. 15.VIII.2018 Konstantinova \& Savchenko K14-2c-18 (KPABG 123724). New to Sørkapp Land.

- Spitsbergen, Wedel Jarlsberg Land: Recherchefjorden. Lagerneset $\left(77^{\circ} 31^{\prime} 20^{\prime \prime} \mathrm{N}, 14^{\circ} 47^{\prime} 14^{\prime \prime} \mathrm{E}\right)$, rock outcrops in wetland on gentle slope, on bare soil. 18.VIII.2018 Konstantinova \& Savchenko K47-3a-18 (KPABG 123667). New to Wedel Jarlsberg Land.

Marchantia polymorpha L. subsp. montivagans Bischl. et Boissel.-Dub.

- Spitsbergen, James Land: Ekmanfjorden. Flintholmen (78 $39^{\prime}$ N, 14³5'E), 17.VII.1973 Frisvoll, det. Konstantinova 2016 (TRH B73661/1). New to James Land.

Marchantia polymorpha L. subsp. ruderalis Bischl. et Boissel.-Dub. - Spitsbergen, Dickson Land: Billefjorden. Pyramiden, south coast of Mimerbukta, the bottom of Yggdrasilkampen Mtn (78 $\left.38^{\prime} 24^{\prime \prime} \mathrm{N}, 16^{\circ} 20^{\prime} 35^{\prime \prime} \mathrm{E}\right)$, anthropogenic site in delta of Mimerselva, between embankment and the north faced slope. Deschampsia-Bryum community overgrow bones, on mosses. 28.VII.2008 Konstantinova K51-08 (KPABG 116557). New to Dickson Land.

Marchantia quadrata Scop.

- Nordaustlandet, Prins Oscars Land: Innvika Bay. South-west coast of Depotlaguna, right bank of Ringgåsdalen River $\left(80^{\circ} 06^{\prime} 46^{\prime \prime} \mathrm{N}, 23^{\circ} 03^{\prime} 40^{\prime \prime} \mathrm{E}\right)$, north facing slope to the river. 23.VII.2011 Konstantinova E303-1-11 (KPABG 115397). New to Prins Oscars Land.

- Spitsbergen, Nathorst Land: Bellsund. Van Keulenfjorden. Strandvollsletta $\left(77^{\circ} 37^{\prime} 23^{\prime \prime} \mathrm{N}, 1^{\circ} 54^{\prime} 16^{\prime \prime} \mathrm{E}\right)$, hollow with huge rocks, bank of small stream, between rocks. 19.VIII.2018 Konstantinova \& Savchenko K55-2b-18 (KPABG 123688). New to Nathorst Land.

- Spitsbergen, Nordenskiöld Land: Adventfjorden. Hanaskogdalen $\left(78^{\circ} 17^{\prime} \mathrm{N}, 15^{\circ} 48^{\prime} \mathrm{E}\right)$, on the south side of the river, 23.VII.1977 Frisvoll, det. Konstantinova 2016 (TRH B73836/ 1). New to Nordenskiöld Land although Prestø et al. (2014) mentioned its occurrence there.

Marchantia quadrata Scop. subsp. hyperborea (R.M.Schust.) Borovich.

- Spitsbergen, Dickson Land: Dicksonfjorden, innermost part of the fjord on the E side (78 $\left.50^{\prime} \mathrm{N}, 15^{\circ} 25^{\prime} \mathrm{E}\right)$. 5.VIII.1973 Frisvoll, det. Konstantinova 2016 (TRH B73844/1). New to Dickson Land.

— Spitsbergen, Haakon VII Land: Bockfjorden. Trollkjeldene, ved 1. kilde nordfra $\left(79^{\circ} 23^{\prime} \mathrm{N}, 13^{\circ} 27^{\prime} \mathrm{E}\right)$. Late snow patch. 23.VIII.1974 Frisvoll, det. Konstantinova 2016 (TRH B73835/ 1). New to Haakon VII Land.

- Spitsbergen, James Land: Ekmanfjorden. Flintholmen (78³9’N, 14³5’E). 17.VII.1973 Frisvoll, det. Konstantinova 2016 (TRH B73839/1). New to James Land.

- Spitsbergen, Oscar II Land: Trygghamna, Protektorfjellet (78 $\left.{ }^{\circ} 13^{\prime} 59^{\prime \prime} \mathrm{N}, 13^{\circ} 49^{\prime} 26^{\prime \prime E}\right), 7 . V I I I .2009$ Borovichev ÁA196-1109 (KPABG 114798). New to Oscar II Land. 
- Prins Karls Forland: North facing slope Fullittknausen Mountain $\left(78^{\circ} 32^{\prime} 08^{\prime \prime} \mathrm{N}, 11^{\circ} 22^{\prime} 13^{\prime \prime} \mathrm{E}\right)$, cliffs under bird colony, on soil and peat. 6.VIII.2016 Savchenko SA16-36-2a (KPABG 121477). New to Prins Karls Forland.

Marchantia romanica (Radian) D.G.Long, Crand.-Stotl., L.L.Forrest et J.C.Villarreal

- Spitsbergen, Haakon VII Land: Reinsdyrflya, south of Velkomstvarden $\left(79^{\circ} 50^{\prime} \mathrm{N}, 13^{\circ} 48^{\prime} \mathrm{E}\right), 20 . \mathrm{VIII} .1974$ Frisvoll, det. Konstantinova 2016 (TRH B73834/1). New to Haakon VII Land.

- Spitsbergen, Oscar II Land: Brøggerhalvøya, innermost of

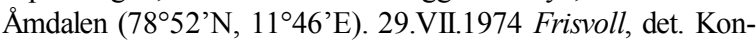
stantinova 2016 (TRH B73843/1). New to Oscar II Land.

Marsupella condensata (Ångstr. ex C.Hartm.) Lindb. ex Kaal.

- Nordaustlandet, Prins Oscars Land: east coast of Rijp Fjorden, north faced cliffs of Bluffvarden Mount on southern bank of Heieren Lake $\left(80^{\circ} 12^{\prime} 39^{\prime \prime} \mathrm{N}, 2^{\circ} 28^{\prime} 31^{\prime \prime} \mathrm{E}\right)$, slope mount, on muddy rocks in mossy bed of temperate stream. 2006.VII.04 Konstantinova \& Savchenko K78-1f-15 (KPABG 122999). New to Nordaustlandet.

Mesoptychia badensis (Gottsche ex Rabenh.) L.Söderstr. et Váňa

- Spitsbergen, Dickson Land: Nordfjorden. Kapp Wijk, on the shore close to Oxaashytta (78³4'N, 15¹4'E), 30.VI.1973 Frisvoll, det. Konstantinova 2016 (TRH B74044/1). New to Dickson Land.

- Spitsbergen, Sørkapp Land: Stormbukta $\left(76^{\circ} 43^{\prime} 05^{\prime \prime N}\right.$, $\left.16^{\circ} 18^{\prime} 46^{\prime \prime} \mathrm{E}\right)$, the gentle slope of the northern exposure to the stream valley, the seepage gravely soil, between small rocks. 14.VIII.2018 Konstantinova \& Savchenko K8-3-18 (KPABG 123552). New to Sørkapp Land.

Mesoptychia collaris (Nees) L.Söderstr. et Váňa

— Spitsbergen, Dickson Land: Billefjorden. Pyramiden, northern coast of Mimerbukta, the bottom of Pyramida Mtn. (78³9'34"N, $16^{\circ} 24^{\prime} 52$ "E), south-east facing slope, Cassiope-Dryas-lichen community with many stones, on spot of fine earth, between small rocks. 25.VII.2008 Konstantinova K22-2-08 (KPABG 118856)]. New to Dickson Land.

- Spitsbergen, Haakon VII Land: Bockfjorden. At the end of Karlsbremorena inland of Trollkjeldene $\left(79^{\circ} 23^{\prime} 51^{\prime \prime N}\right.$, $13^{\circ} 27^{\prime} 59^{\prime \prime E}$ ), wet sand at a stream. 13.VIII.1974 Frisvoll 74050 a (KPABG 122652). New to Haakon VII Land.

- Spitsbergen, Sørkapp Land: Stormbukta $\left(76^{\circ} 42^{\prime} 53^{\prime \prime N}\right.$, $16^{\circ} 19^{\prime} 02^{\prime \prime E}$ ), hollow, the bed of a temporary watercourse, at base of rock. 14.VIII.2018 Konstantinova \& Savchenko K5-1a18 (KPABG 123543). New to Sørkapp Land.

Mesoptychia gillmanii (Austin) L.Söderstr. et Váňa

- Spitsbergen, Wedel Jarlsberg Land: Dunderbukta, Dunderdalen northern part $\left(77^{\circ} 28^{\prime} 45^{\prime \prime} \mathrm{N}, 14^{\circ} 01^{\prime} 53\right.$ "E), Ptilidium dominated tundra, on loamy soil on nonsorted circle. 16.VIII.2018 Konstantinova \& Savchenko K20-3b-18 (KPABG 123614). New to Wedel Jarlsberg Land.

Mesoptychia heterocolpos (Thed. ex Hartm.) L.Söderstr. et Váňa var. heterocolpos

- Spitsbergen, Nathorst Land: Bellsund. Van Keulenfjorden. Strandvollsletta $\left(77^{\circ} 37^{\prime} 22^{\prime \prime} \mathrm{N}, 1^{\circ} 54^{\prime} 18^{\prime \prime} \mathrm{E}\right)$, steep slope, south exposure covered by mosses with scattered Oxyria digyna, Polygonum viviparum, Saxifraga oppositifolia, between gravel on loamy soil. 19.VIII.2018 Konstantinova \& Savchenko K50$3 d-18$ (KPABG 123730). New to Nathorst Land.

Mesoptychia heterocolpos var. arctica (S.W.Arnell) L.Söderstr. et Váña.

- Nordaustlandet, Gustav V Land: Murchison Fjord, Kinnvika, west slope of Raudstupet Mtn. (8002’38'N, 18³3’23"E),
24.VII.1910 Konstantinova K9-6-10 (KPABG 114688). New to Nordaustlandet.

- Spitsbergen, Dickson Land: Billefjorden. Pyramiden, middle part of Mimerdalen, right bank of Mimerelva $\left(78^{\circ} 38^{\prime} 40^{\prime \prime} \mathrm{N}\right.$, $\left.16^{\circ} 08^{\prime} 57^{\prime \prime} \mathrm{E}\right)$, huge depression at the bottom of second river terrace, on left side of road. În soil $(1-3 \mathrm{~cm})$ on side of rock on slope. 2.VIII.2008 Konstantinova K92-4b-08 (KPABG 116590). New to Dickson Land.

Mesoptychia heterocolpos var. harpanthoides (Bryhn et Kaal.) L.Söderstr. et Váňa

- Nordaustlandet, Prins Oscars Land: Innvika Bay, south-west coast of Depotlaguna, right bank of Ringgåsdalen River $\left(80^{\circ} 06^{\prime} 46^{\prime \prime} \mathrm{N}, 23^{\circ} 03^{\prime} 40^{\prime \prime} \mathrm{E}\right)$, north facing slope to the river. 23.VII.2011 Konstantinova K303-2-11 (KPABG 115398). New to Nordaustlandet.

— Spitsbergen, Dickson Land: Billefjorden. Pyramiden, middle part of Mimerdalen, right bank of Mimerelva $\left(78^{\circ} 38^{\prime} 40^{\prime \prime} \mathrm{N}\right.$, $\left.16^{\circ} 08^{\prime} 57^{\prime \prime} \mathrm{E}\right)$, huge depression at the bottom of second river terrace, on left side of road. On rocks between mosses. 2.VIII.2008 Konstantinova K90-2-08 (KPABG 118865). New to Dickson Land.

- Spitsbergen, Nathorst Land: Bellsund. Van Keulenfjorden.

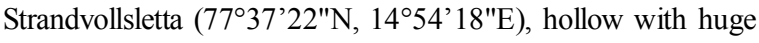
rocks and small stream, rock field at base of slope, on rock. 19.VIII.2018 Konstantinova \& Savchenko K57-2a-18 (KPABG 123721)].

- Spitsbergen, Nordenskiöld Land: Adventdalen, Gruve 7 fjellet ( $\left.78^{\circ} 09^{\prime} 48^{\prime \prime} \mathrm{N}, 16^{\circ} 04^{\prime} 58^{\prime \prime} \mathrm{E}\right)$. Steep snowbed community. 27.VII.2013 Hassel, det. Konstantinova 2016 (TRH B37527/ 2). New to Nordenskiöld Land.

- Spitsbergen, Ny-Friesland: Ringhorndalen, Dellingstupa. In the mossy run-off next to the Cassiope heath. 13.VIII.2015 Bronken Eidesen, det. Söderström 2021 (TRH B38901/2). New to NyFriesland.

- Spitsbergen, Sørkapp Land: South coast Hornsund Bay, at the base of Krykkjestupet (76 $\left.57^{\prime} 54^{\prime \prime} \mathrm{N}, 15^{\circ} 59^{\prime} 33^{\prime \prime} \mathrm{E}\right)$, seeping cliffs under bird colonies, on humus and fine earth between rocks. 15.VIII.2018 Konstantinova \& Savchenko K18-1-18 (KPABG 123478). New to Sørkapp Land.

- Spitsbergen, Wedel Jarlsberg Land: Dunderbukta, Dunderdalen, northern part $\left(77^{\circ} 28^{\prime} 47^{\prime \prime} \mathrm{N}, 14^{\circ} 02^{\prime} 20^{\prime \prime} \mathrm{E}\right)$, at base of slope, on huge rock covered by Ptilidium and lichens. 16.VIII.2018 Konstantinova \& Savchenko K28-1a-18 (KPABG 123628). New to Wedel Jarlsberg Land.

Mesoptychia sahlbergii (Lindb. et Arnell) A.Evans

— Spitsbergen, Nordenskiöld Land: Sassendalen, 2-3 km SSE of Fredheim $\left(78^{\circ} 18^{\prime} \mathrm{N}, 17^{\circ} 00^{\prime} \mathrm{E}\right)$, along a SW exposed, small spring on limestone with Carex saxatilis, Fissidens adianthoides \& Meesia triquetra. 12.VIII1986 Elvebakk 86:558 (TROM 410381/ 1). New to Nordenskiöld Land.

Nardia geoscyphus (De Not.) Lindb.

- Spitsbergen, Heer Land: Van Mijenfjorden. Rindersbukta. Ot-

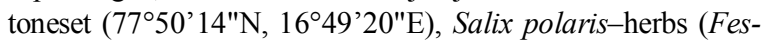
tuca arctica, Bistorta viviparum, Oxyria digyna) with Scapania obcordata dominated in the ground cover, on gentle slope, on muddy ground. 17.VIII.2018 Konstantinova \& Savchenko K35-2-18 (KPABG 123640). New to Heer Land.

Neoorthocaulis hyperboreus (R.M.Schust.) L.Söderstr., De Roo et Hedd. New to Svalbard.

- Spitsbergen, Albert I Land: Lilliehöökfjorden. Ved Hajern

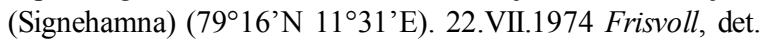
Konstantinova 2016, conf. Söderström \& Hassel 2021 (TRH B74085/1). 
- Spitsbergen, Dickson Land: Sauriedalen, Ibsenfjella V (78³3’06"N, 15³2’30"E), 31.VII.1973 Frisvoll 74084 (KPABG 122647).

- Spitsbergen, Nathorst Land: Bellsund. Van Keulenfjorden. Strandvollsletta $\left(77^{\circ} 37^{\prime} 22^{\prime \prime} \mathrm{N}, 1^{\circ} 54^{\prime} 18^{\prime \prime} \mathrm{E}\right)$, hollow with huge rocks and small stream, rock field at base of slope, on rock. 19.VIII.2018 Konstantinova \& Savchenko K57-1-18 (KPABG 123691)].

- Spitsbergen, Nordenskiöld Land, Bjørndalen, on the E side, c. $500 \mathrm{~m}$ from the sea, in rich fen, $25 \mathrm{~m}$ a.s.1. $\left(78^{\circ} 13^{\prime} \mathrm{N}, 15^{\circ} 19^{\prime \prime} \mathrm{E}\right)$, 19.VII.1977 Frisvoll, det. Konstantinova 2016, conf. Söderström \& Hassel 2021 (TRH B73810)

- Prins Karls Forland: South-east slope of Alfred Larsentoppen Mountain $\left(78^{\circ} 32^{\prime} 59^{\prime \prime} \mathrm{N}, 11^{\circ} 11^{\prime} 58^{\prime \prime} \mathrm{E}\right)$, on overgrowing plot of slope. 8.VIII.2016 Savchenko SA16-44-1c (KPABG 121526).

- Spitsbergen, Sørkapp Land: Stormbukta $\left(76^{\circ} 43^{\prime} 11^{\prime \prime N}\right.$, $16^{\circ} 18^{\prime} 06^{\prime \prime E}$ ), boggy coastal glacial terrace, at base of cliff, under rock, on rock. 14.VIII.2018 Konstantinova \& Savchenko K11-1d-18 (KPABG 123570)].

- Spitsbergen, Wedel Jarlsberg Land: Dunderbukta, Dunderdalen, northern part $\left(77^{\circ} 28^{\prime} 48^{\prime \prime} \mathrm{N}, 14^{\circ} 02^{\prime} 10^{\prime \prime} \mathrm{E}\right)$, earth hummocks at base of gentle slope, on waterlogged soil. 16.VIII.2018 Konstantinova \& Savchenko K21-2-18 (KPABG 123616)

Odontoschisma elongatum (Lindb.) A.Evans

- Nordaustlandet, Prins Oscars Land: Innvika Bay. North-west coast of Depotlaguna, left bank of Ringgåsdalen River $\left(80^{\circ} 07^{\prime} 00^{\prime \prime} \mathrm{N}, 23^{\circ} 03^{\prime} 23^{\prime \prime E}\right)$, valley of rocky stream on terrace. 30.VII.1911 Konstantinova K336-2a-11 (KPABG 115441), New to Nordaustlandet.

Odontoschisma macounii (Austin) Underw.

- Nordaustlandet, Gustav V Land: Laponiahalvøya, Depotvika (80²3’N 1943'E), Phippsia algida snow patch, 20.VIII.1979 Brattbakk (TRH B19085). New to Nordaustlandet.

- Nordaustlandet, Prins Oscars Land: The valley of an unnamed lake between Bluffvarden and the Sanfordhøgdene hill (80¹3’05"N, 22²9'39"E). 3.VIII.2006 Konstantinova K65-306 (KPABG 111780).

- Spitsbergen, Wedel Jarlsberg Land: Recherchefjorden. Lagerneset $\left(77^{\circ} 31^{\prime} 32^{\prime \prime} \mathrm{N}, 14^{\circ} 45^{\prime} 54^{\prime \prime} \mathrm{E}\right)$, river terrace, patterned ground tundra on gentle slope, on frost hollows, in crevices. 18.VIII.2018 Konstantinova \& Savchenko K45-1b-18 (KPABG 123657). New to Wedel Jarlsberg Land.

Peltolepis quadrata (Saut.) Müll.Frib.

- Spitsbergen, Nathorst Land: Bellsund. Van Keulenfjorden. Strandvollsletta $\left(77^{\circ} 37^{\prime} 22^{\prime \prime} \mathrm{N}, 1^{\circ} 54^{\prime} 18^{\prime \prime} \mathrm{E}\right)$, steep slope, south exposure covered by mosses with scattered Oxyria digyna, Polygonum viviparum, Saxifraga oppositifolia, between gravel on loamy soil. 19.VIII.2018 Konstantinova \& Savchenko K505a-18 (KPABG 123680)]. New to Nathorst Land.

- Spitsbergen, Sørkapp Land: Stormbukta $\left(76^{\circ} 43^{\prime} 11^{\prime \prime N}\right.$, $\left.16^{\circ} 18^{\prime} 06^{\prime \prime} \mathrm{E}\right)$, the gentle slope of the northern exposure to the stream valley, the seepage gravelly soil, between small rocks. 14.VIII.2018 Konstantinova \& Savchenko K8-3-18 (KPABG 123552). New to Sørkapp Land.

- Spitsbergen, Wedel Jarlsberg Land: Dunderbukta, Dunderdalen northern part $\left(77^{\circ} 28^{\prime} 48^{\prime \prime} \mathrm{N}, 14^{\circ} 02^{\prime} 10^{\prime \prime} \mathrm{E}\right)$, Ptilidium dominated tundra, on loamy soil on nonsorted circle. 16.VIII.2018 Konstantinova \& Savchenko K20-2c-18 (KPABG 123610). New to Wedel Jarlsberg Land.

Ptilidium ciliare (L.) Hampe.

— Nordaustlandet, Prins Oscars Land: Bluffvarden $\left(80^{\circ} 12^{\prime} 40^{\prime \prime} \mathrm{N}\right.$, $\left.22^{\circ} 27^{\prime} 39^{\prime \prime} \mathrm{E}\right)$, the slope of the north-western exposure.
6.VIII.2006 Konstantinova E109-1ŕ-06 (KPABG 111802). New to Prins Oscars Land.

— Spitsbergen, Andrée Land: Jakobsenbukta ( $\left.79^{\circ} 36^{\prime} \mathrm{N}, 14^{\circ} 12^{\prime} \mathrm{E}\right)$, 12.VIII.1958 Rønning, det. Frisvoll 1973 (TRH B74897/5). New to Andrée Land.

- Spitsbergen, Dickson Land: Nordfjorden, at Hagahytta (78²9’N, 15²0’E), 9.VII.1973 Frisvoll (TRH B73852/1). New to Dickson Land although mentioned as occurring the by Pyramiden by Belkina et al. (2015) without any further details.

— Spitsbergen, James Land: Ekmanfjorden. Flintholmen (78³9’ N 14³'E), 17.VII.1973 Frisvoll (TRH B73849/1). New to James Land.

Riccardia chamedryfolia (With.) Grolle

- Spitsbergen, Nordenskiöld Land, Bolterdalen, partly dead Sphagnum hummock (78 $\left.09^{\prime} 03^{\prime \prime} \mathrm{N}, 15^{\circ} 59^{\prime} 18^{\prime \prime} \mathrm{E}\right), 114 \mathrm{~m}$ a.s.l. 21.VIII.2019 Hassel (TRH B116276/1). Genus new to Svalbard.

- Spitsbergen, Nordenskiöld Land, Colesbukta, peaty soil on moist tundra, together with Schistochilopsis opacifolia (78 $\left.07^{\prime} 21^{\prime \prime} \mathrm{N}, 15^{\circ} 01^{\prime} 02^{\prime \prime} \mathrm{E}\right)$. 23.VIII.2019 Hassel (TRH B695465/1). NOTE: The nearest known locality is in northern Norway. The specimens were typical Riccardia chamedryfolia with some shoots tri-pinnate and with a large oilbody in all cells.

Saccobasis polita (Nees) H.Buch

- Spitsbergen, Haakon VII Land: Bockfjorden. Trollkjeldene ( $\left.79^{\circ} 23^{\prime} \mathrm{N} 13^{\circ} 27^{\prime} \mathrm{E}\right)$, svovelkjelder, 5.VIII.1974 Frisvoll (TRH B74194/1). New to Haakon VII Land.

- Spitsbergen, Nordenskiöld Land: Adventdalen, Longyearbyen, Nybyen by "Huset" $\left(78^{\circ} 12^{\prime} 29^{\prime \prime N}, 15^{\circ} 35^{\prime} 22^{\prime \prime E}\right)$, wet part in Cassiope heath, 22.VII. 2013 Hassel (TRH B37352/1). New to Nordenskiöld Land.

Saccobasis polymorpha (R.M.Schust.) Schljakov.

- Spitsbergen, Sørkapp Land: Stormbukta $\left(76^{\circ} 43^{\prime} 11\right.$ "N, $16^{\circ} 18^{\prime} 06^{\prime \prime E)}$ boggy coastal glacial terrace, at base of cliff, under rock, on rock. 14.VIII.2018 Konstantinova \& Savchenko K11-1a-18 (KPABG 123564). New to Sørkapp Land.

Sauteria alpina (Nees) Nees.

- Spitsbergen, Bünsow Land: Gipsdalen, middle part, to $\mathrm{S}$ of river $\left(78^{\circ} 30^{\prime} \mathrm{N} 16^{\circ} 52^{\prime} \mathrm{E}\right)$, snow-bed, below ridge, on calcareous silt. 18.VII.1985 Engelskjøn (TROM 340446/1). New to Bünsow Land.

- Spitsbergen, James Land: Ekmanfjorden. Flintholmen (78³9’ N 14³5"'E), 17.VII.1973 Frisvoll, det. N.A. Konstantinova 2016 (TRH B73908/1). New to James Land.

- Spitsbergen, Nordenskiöld Land: Van Mijenfjorden, Braganzavågen (77 $\left.55^{\prime} \mathrm{N}, 16^{\circ} 51^{\prime} \mathrm{E}\right)$. 6.VIII.1986 Schwenke \& Øvstedal (TROM 340417/1). New to Nordenskiöld Land.

Scapania cuspiduligera (Nees) Müll.Frib.

- Nordaustlandet, Prins Oscars Land: Innvika Bay, south-west coast of Depotlaguna, right bank of Ringgåsdalen River $\left(80^{\circ} 07^{\prime} 00^{\prime \prime} \mathrm{N}, 23^{\circ} 03^{\prime} 23^{\prime \prime} \mathrm{E}\right)$, north facing slope to the river. 23.VII.2011 Konstantinova K303-1-11 (KPABG 115397). New to Nordaustlandet.

- Spitsbergen, Nathorst Land: Bellsund. Van Keulenfjorden. Strandvollsletta $\left(77^{\circ} 37^{\prime} 22^{\prime \prime} \mathrm{N}, 14^{\circ} 54^{\prime} 18^{\prime \prime} \mathrm{E}\right)$, hollow with huge rocks and small stream, rock field at base of slope, on rock. 19.VIII.2018 Konstantinova \& Savchenko (KPABG 123696). New to Nathorst Land.

- Spitsbergen, Wedel Jarlsberg Land: Dunderbukta, Dunderdalen, northern part $\left(77^{\circ} 28^{\prime} 48^{\prime \prime} \mathrm{N}, 14^{\circ} 02^{\prime} 10^{\prime \prime} \mathrm{E}\right)$, on side of moss covered rock, on rock. 16.VIII.2018 Konstantinova \& Savchenko 
K26-1-18 (KPABG 123623). New to Wedel Jarlsberg Land. Scapania gymnostomophila Kaal.

- Spitsbergen, Nathorst Land: Bellsund. Van Keulenfjorden. Strandvollsletta $\left(77^{\circ} 37^{\prime} 22^{\prime \prime} \mathrm{N}, 14^{\circ} 54^{\prime} 18^{\prime \prime} \mathrm{E}\right)$, hollow with huge rocks and small stream, rock field at base of slope, on rock. 19.VIII.2018 Konstantinova \& Savchenko K57-1-18 (KPABG 123691). New to Nathorst Land.

Scapania kaurinii Ryan.

- Nordaustlandet, Prins Oscars Land: The plain between Bluffvarden and an unnamed large river that flows into the Rijp Fjord (80¹2'04"N, 22³1'45"E). 6.VIII.2006 Konstantinova K1131-06 (KPABG 111809). New to Nordaustlandet.

Scapania ligulifolia R.M.Schust.

- Nordaustlandet, Prins Oscars Land: Innvika Bay, south-west coast of Depotlaguna, right bank of Ringgåsdalen River $\left(80^{\circ} 07^{\prime} 00^{\prime \prime} \mathrm{N}, 23^{\circ} 03^{\prime} 23^{\prime \prime} \mathrm{E}\right)$, north facing slope to the river. 23.VII.2011 Konstantinova K303-1-11 (KPABG 115397). New to Prins Oscars Land.

- Spitsbergen, Nathorst Land: Bellsund. Van Keulenfjorden. Strandvollsletta $\left(77^{\circ} 37^{\prime} 22^{\prime \prime} \mathrm{N}, 1^{\circ} 54^{\prime} 18^{\prime \prime} \mathrm{E}\right)$, steep slope, south exposure covered by mosses with scattered Oxyria digyna, Polygonum viviparum, Saxifraga oppositifolia, between gravel on loamy soil. 19.VIII.2018 Konstantinova \& Savchenko K503b-18 (KPABG 123678). New to Nathorst Land.

- Spitsbergen, Sørkapp Land: Stormbukta $\left(76^{\circ} 42^{\prime} 55^{\prime \prime N}\right.$, $16^{\circ} 18^{\prime} 42^{\prime \prime E}$ ), under rock on the slope of the northern exposition. 14.VIII.2018 Konstantinova \& Savchenko K4-2-18 (KPABG 123542). New to Sørkapp Land.

— Spitsbergen, Wedel Jarlsberg Land: Recherchefjorden. Lagerneset $\left(77^{\circ} 31^{\prime} 08^{\prime \prime} \mathrm{N}, 14^{\circ} 48^{\circ} 09^{\prime \prime} \mathrm{E}\right)$, gentle slope with numerous rivulets and moss mats, on side of hummock. 18.VIII.2018 Konstantinova \& Savchenko K49-4a-18(KPABG 123740). New to Wedel Jarlsberg Land.

Scapania obcordata (Berggr.) S.W.Arnell

- Spitsbergen, Dickson Land: Billefjorden. Pyramiden, Mimerdalen, extreme southeastern part of Odinfjellet, Planteryggen

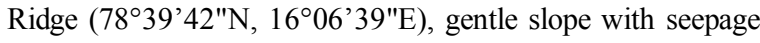
and brooks, dry Salix polaris-Dryas- moss-lichen community with many crevices, in crevices and on under crust of lichens. 30.VII.2008 Konstantinova K71-1a-08 (KPABG 116567). New to Dickson Land.

- Spitsbergen, Haakon VII Land: Bockfjorden, plateaux east on Trolltindane, 900 m (79²2’N, 13¹9'E), 16.VIII.1974 Frisvoll, det Konstantinova 2016 (TRH B73985/1). New to Haakon VII Land.

- Spitsbergen, Heer Land: Van Mijenfjorden. Rindersbukta. Ottoneset $\left(77^{\circ} 50^{\prime} 14^{\prime \prime} \mathrm{N}, 16^{\circ} 49^{\prime} 20^{\prime \prime} \mathrm{E}\right)$, solifluction mantle (slope with numerous rocks and loamy soil), on loamy soil. 17.VIII.2018 Konstantinova \& Savchenko (KPABG 123635). New to Heer Land.

- Spitsbergen, James Land: Blomesletta (78³8’N 1452’E), volcanic rock on the plain. 28.VII.1973 Frisvoll, det. Konstantinova 2016 (TRH B73940/1). New to James Land.

Scapania paludicola Loeske et Müll.Frib.

— Nordaustlandet, Prins Oscars Land: Innvika Bay, north-west coast of Depotlaguna, left bank of Ringgåsdalen River $\left(80^{\circ} 06^{\prime} 19^{\prime \prime} \mathrm{N}, 23^{\circ} 01^{\prime} 50^{\prime \prime} \mathrm{E}\right)$, valley of rocky stream on terrace. 30.VII.2011 Konstantinova K336-1a-11 (KPABG 115440). New to Nordaustlandet.

- Spitsbergen, Nathorst Land: Bellsund. Van Keulenfjorden. Strandvollsletta $\left(77^{\circ} 37^{\prime} 22^{\prime \prime} \mathrm{N}, 14^{\circ} 54^{\prime} 18^{\prime \prime} \mathrm{E}\right)$, steep slope, south exposure covered by mosses with scattered Oxyria digyna,
Polygonum viviparum, Saxifraga oppositifolia, between gravel on loamy soil. 19.VIII.2018 Konstantinova \& Savchenko K503a-18 (KPABG 123677). New to Nathorst Land.

Scapania praetervisa Meyl.

- Spitsbergen, Dickson Land: Nordfjorden. Kapp Wijk, ravine in Kongressfjellet E of Oxaashytta (78 $\left.35^{\prime} \mathrm{N}, 15^{\circ} 16^{\prime} \mathrm{E}\right)$, 5.VII.1973 Frisvoll (TRH B73963/1). New to Dickson Land.

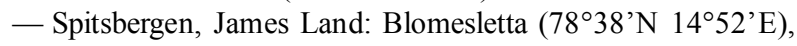
volcanic rock on the plain, 28.VII.1973 Frisvoll (TRH B73964/ 1). New to James Land.

- Spitsbergen, Nordenskiöld Land: Kapp Linné (7804’N, $\left.13^{\circ} 37^{\prime} \mathrm{E}\right)$, inland from the radio station, 1.VII.1974 Frisvoll (TRH B73966/1). New to Nordenskiöld Land.

Scapania tundrae (Arnell) H.Buch

- Nordaustlandet: Prins Oscars Land: Innvika Bay, south-west coast of Depotlaguna, the bottom of western slope of Vikvaktaren $\left(77^{\circ} 50^{\prime} 14^{\prime \prime} \mathrm{N}, 23^{\circ} 05^{\prime} 13^{\prime \prime} \mathrm{E}\right)$, huge boulders with numerous rivulets. 24.VII.2011 Konstantinova K306-12-11 (KPABG 115406). New to Nordaustlandet although mentioned to occur there without further details by Konstantinova \& Savchenko (2015b).

- Spitsbergen, Haakon VII Land: Bockfjorden, top of Trolltindane (79²2’N, 1316'E). 16.VIII.1974 Frisvoll, det. Konstantinova 2016 (TRH B73948/1). New to Haakon VII Land.

Schljakovia kunzeana (Huebener) Konstant. et Vilnet

- Spitsbergen, Dickson Land: Sauriedalen, Ibsenfjella V (78 33’N, 15³3’E), 31.VII.1973 Frisvoll, det. Konstantinova 2016 (TRH B74084/1). New to Dickson Land.

- Spitsbergen, Nathorst Land: Bellsund. Van Keulenfjorden. Strandvollsletta $\left(77^{\circ} 37^{\prime} 22^{\prime \prime} \mathrm{N}, 1^{\circ} 54^{\prime} 18^{\prime \prime E}\right)$, hollow with huge rocks. 19.VIII.2018 Konstantinova \& Savchenko K51-1-18 (KPABG 123682). New to Nathorst Land.

— Spitsbergen, Sørkapp Land: Stormbukta $\left(76^{\circ} 42^{\prime} 55^{\prime \prime N}\right.$, $16^{\circ} 18^{\prime} 42^{\prime \prime E}$ ), hollow, the bed of a temporary watercourse, at base of rock. 14.VIII.2018 Konstantinova \& Savchenko K5-1b18 (KPABG 123560). New to Sørkapp Land.

- Spitsbergen, Wedel Jarlsberg Land: Dunderbukta, Dunderdalen northern part $\left(77^{\circ} 28^{\prime} 48^{\prime \prime N}, 1^{\circ} 02^{\prime} 10^{\prime \prime} \mathrm{E}\right)$, Ptilidium dominated tundra, in crevices on nonsorted circle. 16.VIII.2018 Konstantinova \& Savchenko K20-1a-18 (KPABG 123606). New to Wedel Jarlsberg Land.

Schljakovianthus quadrilobus (Lindb.) Konstant. et Vilnet

— Spitsbergen, Andrée Land: Jacobsenbukta (79³6’ N, 14 $\left.{ }^{\circ} 12^{\prime} \mathrm{E}\right)$, 12.VIII.1958 Rønning, det. Frisvoll 1976 (TRH B74555/5). New to Andrée Land.

- Spitsbergen, James Land: Dicksonfjorden SV, in the valley between Trollfuglfjella and Tolmodryggen $\left(78^{\circ} 40^{\prime} \mathrm{N}, 14^{\circ} 58^{\prime} \mathrm{E}\right)$, 27.VII.1973 Frisvoll (TRH B73805/1). New to James Land.

- Spitsbergen, Nathorst Land: Bellsund. Van Keulenfjorden. Strandvollsletta $\left(77^{\circ} 37^{\prime} 22^{\prime \prime} \mathrm{N}, 14^{\circ} 54^{\prime} 18^{\prime \prime} \mathrm{E}\right)$, hollow with huge rocks and small stream, rock field at base of slope, on rock. 19.VIII.2018 Konstantinova \& Savchenko K58-1e-18 (KPABG 123696)].

— Spitsbergen, Sørkapp Land: Stormbukta $\left(76^{\circ} 42^{\prime} 55^{\prime \prime N}\right.$, $16^{\circ} 18^{\prime} 42^{\prime \prime E}$ ), hollow, the bed of a temporary watercourse, at base of rock. 14.VIII.2018 Konstantinova \& Savchenko K5-1a18 (KPABG 123543). New to Sørkapp Land.

Solenostoma confertissimum (Nees) Schljakov

- Spitsbergen, Nordenskiöld Land: The eastern shore of the Grøndalen Valley (7801'25"N, 14²2'33"E). 24.VII.2004 Konstantinova \& Savchenko K108-2-04 (KPABG 109946). New to Nordenskiöld Land. 
Solenostoma obovatum (Nees) C.Massal.

- Spitsbergen, Dickson Land: Billefjorden SV, near Svenskehuset $\left(78^{\circ} 29^{\prime} \mathrm{N}, 15^{\circ} 42^{\prime} \mathrm{E}\right), 19$.VIII.1973 Frisvoll, det. Konstantinova 2016 (TRH B74038/1). New to Dickson Land.

- Spitsbergen, Nathorst Land: Bellsund. Van Keulenfjorden. Strandvollsletta $\left(77^{\circ} 37^{\prime} 22^{\prime \prime} \mathrm{N}, 1^{\circ} 54^{\prime} 18^{\prime \prime E}\right)$, hollow with huge rocks. 19.VIII.2018 Konstantinova \& Savchenko K51-1-18 (KPABG 123682). New to Nathorst Land.

- Spitsbergen, Sørkapp Land: Stormbukta $\left(76^{\circ} 42^{\prime} 55^{\prime \prime N}\right.$, $\left.16^{\circ} 18^{\prime} 42^{\prime \prime} \mathrm{E}\right)$ in crevices at the base of the outliers. 14.VIII.2018 Konstantinova \& Savchenko K2-1b-18 (KPABG 123540). New to Sørkapp Land.

- Spitsbergen, Wedel Jarlsberg Land: Dunderbukta, Dunderdalen northern part $\left(77^{\circ} 28^{\prime} 48^{\prime \prime} \mathrm{N}, 14^{\circ} 02^{\prime} 10^{\prime \prime} \mathrm{E}\right)$, Ptilidium dominated tundra, on loamy soil on nonsorted circle. 16.VIII.2018 Konstantinova \& Savchenko K20-2b-18 (KPABG 123609). New to Wedel Jarlsberg Land.

Solenostoma sphaerocarpum (Hook.) Steph.

- Spitsbergen, Dickson Land: Billefjorden. Pyramiden, middle part of Mimerdalen, southern bank of lake $\left(78^{\circ} 38^{\prime} 23^{\prime \prime} \mathrm{N}\right.$, $16^{\circ} 07^{\prime} 31 \mathrm{E}$ ), solifluction slope, on clay along small brook. 2.VIII.2008 Konstantinova K99-1b-08 (KPABG 116601). New to Dickson Land.

- Spitsbergen, Nordenskiöld Land: The eastern shore of the Grøndalen Valley (7801'51"N, 14²0’38"E). 25.VII.2004 Konstantinova \& Savchenko K119-1-04 (KPABG 109986). New to Nordenskiöld Land.

Sphenolobus minutus (Schreb.) Berggr.

— Kvitøya: Giles Land (80¹0’N, 32³0’E), 19.VIII.1898 Andersson \& Hesselman (TRH B673383/1). New to Kvitøya.

- Spitsbergen, Dickson Land: Dicksonfjorden SE. Heimenfjellet $\mathrm{V}, \mathrm{NE}$ of a deep ravine ( $\left.78^{\circ} 35^{\prime} \mathrm{N} 15^{\circ} 27^{\prime} \mathrm{E}\right)$, 30.VII.1973 Frisvoll (TRH B74270/1). New to Dickson Land.

- Spitsbergen, James Land: Ekmanfjorden, Flintholmen (78³9’N, 14³5’E), 17.VII.1973 Frisvoll (TRH B74268). New to James Land.

- Spitsbergen, Ny-Friesland: Mosselhalvøya (79 $\left.56^{\prime} \mathrm{N}, 16^{\circ} 18^{\prime} \mathrm{E}\right)$, under edge of a boulder in a boulderfield. 22.VII.2008 Hagen, det. Prestø 2008 (TRH B38443/1). New to Ny-Friesland.

Tetralophozia setiformis (Ehrh.) Schljakov

— Nordaustlandet, Prins Oscars Land: Innvika Bay $\left(80^{\circ} 07^{\prime} 23^{\prime \prime N}\right.$, $\left.23^{\circ} 02^{\prime} 16^{\prime \prime} \mathrm{E}\right)$. Mountain terrace with snow fields, huge boulders. 30.VII.2011 Konstantinova K340-1-11 (KPABG 115450). New to Prins Oscars Land.

Tritomaria scitula (Taylor) Jørg.

- Spitsbergen, James Land: Blomesletta (783’N 1452’E), late snow patch on the plain, 24.VII.1973 Frisvoll (TRH B74175). New to James Land.

- Spitsbergen, Nathorst Land: Bellsund. Van Keulenfjorden. Strandvollsletta $\left(77^{\circ} 37^{\prime} 22^{\prime \prime} \mathrm{N}, 1^{\circ} 54^{\prime} 18^{\prime \prime} \mathrm{E}\right)$, steep slope, south exposure covered by mosses with scattered Oxyria digyna, Polygonum viviparum, Saxifraga oppositifolia, between gravel on loamy soil. 19.VIII.2018 Konstantinova \& Savchenko K501-18 (KPABG 123675). New to Nathorst Land.

- Spitsbergen, Sørkapp Land: Hornsund Bay, Rasstupet bird cliffs Krykkjestupet ( $\left.76^{\circ} 57^{\prime} 47^{\prime \prime} \mathrm{N}, 15^{\circ} 58^{\prime} 49^{\prime \prime} \mathrm{E}\right)$, at base of large rocky outcrops weakly altered carbonate rocks (seeping cliffs), among mosses. 14.VIII.2018 Konstantinova \& Savchenko K14-2b-18 (KPABG 123723). New to Sørkapp Land.

TYPIFICATION OF TWO NAMES

Original material of two of Lindberg's subspecific names were localized at a visit to $H$. The identity of those names has previously been unclear as the supposed parent species' are rejected from Svalbard.

Jungermannia attenuata (Mårt.) Lindenb. $\beta *$ laxifolia Lindb., Öfvers. Kongl. Vetensk.-Akad. Förh. 23: 560, 1866 (Lindberg 1866). Lectotype (here designated): Svalbard, Albert I Land, "Spitsb., Kobbebay, 1861 M-n [= Malmgren]", H-SOL Barcode H3400237 (http:// id.luomus.fi/HA.H3400237). This specimen is rather typical Trilophozia quinquedentata and is considered syn. nov. to it.

Jungermannia floerkei F.Weber et D.Mohr [I densifolia A major] $\delta$ gemmipara Lindb., Öfvers. Kongl. Vetensk.-Akad. Förh. 23: 560, 1866 (Lindberg 1866), nom. inval. (ICN Art 38.1(a); no description). Original material ("lectotype"): Svalbard, Albert I Land, "Kobbebay", 1861 Malmgren, H-SOL Barcode H3400238 (http://id.luomus.fi/ HA.H3400238). This specimen is a form of Schljakovianthus quadrilobus and is considered syn. nov. to it.

\section{CHECKLIST}

We recognize 105 species of liverworts from Svalbard (Fig. 3). In addition, there are five that are questionable and needs confirmation and 51 that are reported from the archipelago that we reject as error in identification or unlikely to be correctly identified, either because of known distribution and habitat requirements, or because of changed taxonomic understanding. In addition we recognize several infraspecific taxa.

The known number of species has increased considerably the last 25 years from the 85 taxa reported by Frisvoll \& Elvebakk (1996). This number of taxa is rather high compared with other Arctic areas (Daniëls et al. 2013). For instance only 42 species are known from the nearby Franz Josef Islands (Konstantinova et al. 2020c). This relative richness is partly due to the fact that it is one of the best studied (and easiest accessible) areas in the Arctic. Another factor is the relatively warm climate due to the North Atlantic Current as an extension of the Gulf Stream, reaching the western part of the archipelago.

References citing primary reports (citing specimens or material seen by the author) are given in bold. Secondary references not citing any material seen are given in normal font. In cases where we are not aware of any primary source, or the earliest report is a secondary source without any clear connection to a primary source published later, we comment on the situation. The taxonomic value for each here accepted taxon is qualified using a 3-level ranking system as was done in the World Checklist (Söderström et al., 2016). The basic value (default value) is $* *$. In case where a significant proportion of recent publications accept the taxon without comment, it is accepted as a good taxon $(* * *)$. When there is any serious doubts about the value of the taxon in recent literature (e.g. not been studied recently) it is only given one *. Note that this ranking system is global and only refer to the taxonomic value, not to the systematic position of the taxon, or the occurrence in the region. The 
ratings follow Söderström et al. (2016) except in the very few cases where new information is at hand.

In the current checklist we note under which name a taxon is reported except when it is reported under the currently accepted name or a homotypic synonym of it.

\section{Aneura Dumort. \\ *** Aneura pinguis (L.) Dumort. [Riccardia pinguis (L.)} Gray]. SVALBARD: Berggren 1875b, Bryhn 1906, Müller 1954a, Düll 1983, Söderström 1995, Frisvoll \& Elvebakk 1996, Paton 1999, Damsholt 2002, Söderström et al. 2002a, 2002b, Konstantinova 2003, Hodgetts 2015, Hodgetts \& Lockhart 2020. BJøRNøYA: Söderström et al. 2002b. GUSTAV V LAND: Berggren 1875a, Konstantinova \& Savchenko 2012. B $A$ ARENTSøYA: Philippi 1973, Konstantinova \& Savchenko 2018. EDGEØYA: Philippi 1973, Heinemeijer 1979. SPITSBERGEN: Lindberg 1866 as Aneura pinguis a 3 tenuis [Kingsbay]. BüNSOW LAND: Acock 1940. DICKSON LAND: Konstantinova \& Savchenko 2008c, Konstantinova \& Savchenko 2011, Belkina et al. 2015. HAAKON_VII LAND: Lindberg 1866 as Aneura pinguis a 2 minor, Berggren 1875a, Arnell \& Mårtensson 1959, Frisvoll 1978. JAMES LAND: This publication. NATHORST LAND: This publication. NORDENSKIÖLD LAND: Berggren 1875a, Konstantinova \& Savchenko 2008b, Virtanen et al. 2013, Prestø et al. 2014. NYFRIESLAND: Lindberg 1866 as Aneura pinguis a 2 minor. OSCAR II LAND: Arnell \& Mårtensson 1959. SøRKAPP LAND: Dubiel \& Olech 1990. WEEDE_JARLSBERG LAND: RejmentGrochowska 1967, Karczmarz \& Święs 1988, Karczmarz \& Święs 1989, Święs \& Karczmarz 1993b.

Anthelia (Dumort.) Dumort.

** Anthelia juratzkana (Limpr.) Trevis. SVALBARD: Bryhn 1906, Müller 1914a, Frye \& Clark 1943, Müller 1954a, Schuster 1974, Düll 1983, Söderström 1995, Frisvoll \& Elvebakk 1996, Paton 1999, Damsholt 2002, Söderström et al. 2002a, 2002b, Damsholt 2013, Hodgetts 2015, Hodgetts \& Lockhart 2020. BృøRNøYA: Watson 1922, Söderström 1995, Frisvoll \& Elvebakk 1996, Damsholt 2002, Söderström et al. 2002b. GUSTAV V LAND: Konstantinova et al. 2020a. PRINS OSCARS LAND: Konstantinova \& Savchenko 2008a, Konstantinova et al. 2014, 2020b. BARENTSØYA: Philippi 1973, Konstantinova \& Savchenko 2018. EDGEØYA: Philippi 1973. SPITSBERGEN: Gottsche et al. 1847 as Jungermannia julacea $\beta$ clavuligera [Bellsund], Arnell \& Mårtensson 1959 [W. Spitsbergen]. ALBERT I LAND: This publication. DICKSON LAND: Konstantinova \& Savchenko 2008c, Belkina et al. 2015. HAAKON VII LAND: Wegener et al. 1992, Konstantinova \& Savchenko 2006. HEER LAND: This publication. JAMES LAND: This publication. NATHORST LAND: This publication. NORDENSKIÖLD LAND: Eurola 1968, Hadač 1989, Konstantinova \& Savchenko 2007, Thingsgaard \& Damsholt 2007, Konstantinova \& Savchenko 2008a, 2008b, Prestø et al. 2014. OSCAR II LAND: Brattbakk et al. 1978, Kobayashi et al. 1990. PRINS KARLS FORLAND: Ellis et al. 2019c, Konstantinova \& Savchenko 2020a. SØRKAPP LAND: Dubiel \& Olech 1990. WEDEL JARLSBERG LAND: Rejment-Grochowska 1967, Karczmarz \& Święs 1988, Karczmarz \& Święs 1989, Karczmarz \& Święs 1990b, Święs \& Karczmarz 1991a. Arnellia Lindb.

*** Arnellia fennica (Gottsche) Lindb. S Svalbard: Kaalaas 1893b, Bryhn 1906, Arnell 1928, Jørgensen 1934, Frye \& Clark 1943, Steere 1954, Arnell 1956, Schuster 1980, Düll
1983, Söderström 1995, Damsholt 2002, Söderström et al. 2002a, 2002b, Hodgetts 2015, Hodgetts \& Lockhart 2020. BARENTSøYA: Philippi 1973, Frisvoll \& Elvebakk 1996, Konstantinova \& Savchenko 2018. EDGEØYA: Philippi 1973, Frisvoll \& Elvebakk 1996. SPITSBERGEN: Müller 1909b [Kongsfjorden], Arnell \& Mårtensson 1959 [Kongsfjorden]. DICKSON LAND: Frisvoll 1981, Frisvoll \& Elvebakk 1996. HAAKON VII LAND: Frisvoll \& Elvebakk 1996, Konstantinova \& Savchenko 2006, 2008a, 2011. OSCAR II LAND: Berggren 1875a as Southbya fennica, Frisvoll \& Elvebakk 1996, Koroleva \& Borovichev 2014. SABINE LAND: This publication, Frisvoll \& Elvebakk 1996.

Barbilophozia Loeske

*** Barbilophozia barbata (Schmidel ex Schreb.) Loeske. [Jungermannia barbata Schmidel. ex Schreb., Lophozia barbata (Schmidel ex Schreb.) Dumort.]. Svalbard: Düll 1983, Söderström 1995, Paton 1999, Damsholt 2002, Söderström et al. 2002a, Damsholt 2013, Hodgetts 2015, Hodgetts \& Lockhart 2020. GUSTAV V LAND: Berggren 1875a as Jungermannia floerkei var. arcuata. SPITSBERGEN: Gottsche et al. 1847 ["Magdalenen Bay in Bell-Sound (Vahl)"]. ALBERT I LAND: This publication. DICKSON LAND: Frisvoll 1981, Frisvoll \& Blom 1992, Frisvoll \& Elvebakk 1996, Frisvoll \& Blom 1997. HAAKON VII LAND: Frisvoll \& Blom 1992, Frisvoll \& Elvebakk 1996. JAMES LAND: Frisvoll 1981, Frisvoll \& Blom 1997. NORDENSKIÖLD LAND: Hagen 1952, Frisvoll \& Blom 1992, 1997, Frisvoll \& Elvebakk 1996. NOTE: Berggren's (and other early bryologist's) concept of Barbilophozia barbata was very wide and most of the old reports have been (or should be) rejected.

*** Barbilophozia hatcheri (A.Evans) Loeske [Lophozia hatcheri (A.Evans) Steph.]. SvalBARD: Grolle 1960, Schuster 1969, Worley 1972, Düll 1983, Söderström 1995 as, Frisvoll \& Elvebakk 1996, Paton 1999, Bapna \& Kachroo 2000b, Damsholt 2002 as, Söderström et al. 2002a, 2002b, Hentschel et al. 2006, 2007, Vilnet et al. 2010, 2012a, 2012b, Damsholt 2013, Hodgetts 2015, Hodgetts \& Lockhart 2020. B JøRnøYA: Watson 1922, Summerhayes \& Elton 1923a, Arnell 1956, Grolle 1960, Söderström 1995, Frisvoll \& Elvebakk 1996, Damsholt 2002. GustaV_V LAND: TYPE of Jungermannia lycopodioides var. cavifolia, Berggren 1875a as Jungermannia lycopodioides var. cavifolia, Konstantinova \& Savchenko 2012. PRINS OSCARS LAND: This publication. BARENTSØYA: Philippi 1973, Konstantinova \& Savchenko 2018. EDGEØYa: Philippi 1973, Heinemeijer 1979. AlbBert I LAND: Arnell \& Mårtensson 1959, Thingsgaard \& Damsholt 2007. DICKSON LAND: This publication. HAAKON_VII LAND: This publication, Newsham \& GoodallCopestake 2021. HEER LAND: Philippi 1973. NORDENSKIÖLD LAND: Hagen 1952, Konstantinova \& Savchenko 2008a, 2008b. OSCAR II LAND: Arnell \& Mårtensson 1959, Kobayashi et al. 1990, Newsham \& Goodall-Copestake 2021. PRINS KARLS ForLAND: Konstantinova \& Savchenko 2020a. SØRKAPP LAND: Dubiel \& Olech 1990. WEDEL JARLSBERG LAND: Rejment-Grochowska 1967, Karczmarz \& Święs 1988, 1989, Święs \& Karczmarz 1991a, 1993b.

*** Barbilophozia sudetica (Nees ex Huebener) L.Söderstr., De Roo et Hedd. [Lophozia sudetica (Nees ex Huebener) Grolle, Pseudolophozia sudetica (Nees ex Huebener) Konstant. et Vilnet]. SvalBarD: Bryhn 1906 as Lophozia alpestris, Arnell 1928 as Jungermannia alpestris, Harmsen \& Seidenfaden 1932 as Lophozia alpestris, Frye \& Clark 1945 as Lophozia alpestris, Arnell 1956 as Lophozia alpestris, 
Schuster 1969, Worley 1972 as Lophozia alpestris, Düll 1983, Smith 1990, Söderström 1995, Frisvoll \& Elvebakk 1996, Paton 1999, Konstantinova 2000 as Lophozia rufescens, Damsholt 2002, Söderström et al. 2002a, 2002b, 2007 as Lophozia sudetica var. anomala, Vilnet et al. 2010, 2012b both as Pseudolophozia sudetica, Vilnet et al. 2012b as Pseudolophozia debiliformis, Hodgetts 2015, Hodgetts \& Lockhart 2020. B nia alpestris, Watson 1922 as Lophozia alpestris, Summerhayes \& Elton 1923a as Lophozia alpestris, Söderström 1995, Frisvoll \& Elvebakk 1996, Damsholt 2002, Söderström et al. 2002b. GuSTAV V LAND: Berggren 1875a as Jungermannia alpestris. PRINS OSCARS LAND: Konstantinova \& Savchenko 2008a. Barentsøya: Philippi 1973 as Lophozia alpestris. EDGEøya: Philippi 1973 as Lophozia alpestris. KONG KarLS_LAND: Arnell 1900 as Jungermannia alpestris. ALBERT I LAND: Gottsche et al. 1846 as Jungermannia alpestris, Berggren 1875a as Jungermannia alpestris, Arnell \& Mårtensson 1959 as Lophozia alpestris. BüNSOW LAND: Watson 1922 as Lophozia alpestris. DICKSON_LAND: This publication. HAAKON VII LAND: Arnell \& Mårtensson 1959 as Lophozia alpestris, Newsham \& Goodall-Copestake 2021. HeER LAND: Philippi 1973 as Lophozia alpestris. NoRDENSKIÖLD LAND: Berggren 1875a as Jungermannia alpestris, Watson 1922 as Lophozia alpestris, Hadač 1946 as Lophozia alpestris, Arnell \& Mårtensson 1959 as Lophozia alpestris, Hadač 1989 as Lophozia alpestris, Konstantinova \& Savchenko 2007, Konstantinova \& Savchenko 2008a, 2008b. OSCAR II LAND: Arnell \& Mårtensson 1959 as Lophozia alpestris. PRINS KARLS FORLAND: Berggren 1875a as Jungermannia alpestris, Watson 1922 as Lophozia alpestris, Summerhayes \& Elton 1923a, 1923b both as Lophozia alpestris. WEDEL JARLSBERG LAND: Rejment-Grochowska 1967 as Lophozia alpestris, Karczmarz \& Święs 1988, 1989 both as Lophozia alpestris, Święs \& Karczmarz 1991a, 1993b both as Lophozia alpestris.

Blasia L.

*** Blasia pusilla L. Svalbard: Düll 1983, Söderström 1995, Paton 1999, Damsholt 2002, Söderström et al. 2002a, Söderström et al. 2002b, Konstantinova 2003, Hodgetts 2015, Hodgetts \& Lockhart 2020. DiCKSON LAND: Frisvoll 1978, Frisvoll \& Blom 1992, 1997, Frisvoll \& Elvebakk 1996. NORDENSKIÖLD LAND: Frisvoll \& Elvebakk 1996, Frisvoll \& Blom 1997. OSCAR II LAND: Frisvoll \& Blom 1992, 1997, Frisvoll \& Elvebakk 1996.

Blepharostoma (Dumort.) Dumort.

** Blepharostoma brevirete (Bryhn et Kaal.) Vilnet et Bakalin [Blepharostoma trichophyllum var. brevirete Bryhn et Kaal., Blepharostoma trichophyllum subsp. brevirete (Bryhn et Kaal.) R.M.Schust.]. Svalbard: Schuster 1966, Düll 1983, Söderström 1995, Frisvoll \& Elvebakk 1996, Damsholt 2002, Söderström et al. 2002a, 2002b, Damsholt 2013, Hodgetts 2015, Bakalin et al. 2020c, Hodgetts \& Lockhart 2020. BJøRnØYA: Arnell 1951. Schuster 1966, Persson \& Viereck 1983, Söderström 1995, Damsholt 2002, Söderström et al. 2002b, Damsholt 2013. NordaustLandet: Konstantinova \& Savchenko 2015. GUSTAV V LAND: Konstantinova \& Savchenko 2011, 2012, Konstantinova et al. 2014. PRINS OSCARS LAND: Konstantinova \& Savchenko 2008a, Konstantinova et al. 2014. B BARENTSøya: Philippi 1973, Konstantinova et al. 2014, 2018. SPITSBERGEN: Arnell \& Mårtensson 1959 [W. Spitsbergen]. DICKSON_LAND: Konstantinova
\& Savchenko 2008c, 2011. HAAKON VII LAND: Konstantinova \& Savchenko 2006, 2008a, 2011, Thingsgaard \& Damsholt 2007. HeER LAND: Konstantinova et al. 2020b. JAMES LAND: Thingsgaard \& Damsholt 2007. NATHORST LAND: This publication. NORDENSKIÖLD LAND: Konstantinova \& Savchenko 2007, 2008a, 2008b, Stebel et al. 2018, Konstantinova et al. 2020b. NY-FRIESLAND: This publication. OSCAR II LAND: Thingsgaard \& Damsholt 2007, Borovichev 2010. PRINS KARLS FORLAND: Ellis et al. 2019c, Konstantinova \& Savchenko 2020a. S $ø$ RKAPP LAND: This publication. WEDEL JARLSBERG LAND: Rejment-Grochowska 1967, Karczmarz \& Święs 1988, Święs \& Karczmarz 1991a, 1993b. NOTE: We are not aware of any primary report from Bjørnøya unless the report of Blepharostoma trichophyllum by Watson (1922) can be referred there.

\section{Cephalozia (Dumort.) Dumort.}

*** Cephalozia ambigua C.Massal. [Cephalozia bicuspidata subsp. ambigua (C.Massal.) R.M.Schust.]. SvalbarD: Schuster 1974, Düll 1983, Smith 1990, Söderström 1995, Paton 1999, Damsholt 2002, Söderström et al. 2002a, 2002b, Vilnet et al. 2012a, Damsholt 2013, Potemkin 2014, Hodgetts 2015, Feldberg et al. 2016, Hodgetts \& Lockhart 2020. BJØRNØYA: Söderström 1995, Frisvoll \& Elvebakk 1996 [with doubt], Damsholt 2002, Söderström et al. 2002b. GustaV V LAND: Konstantinova \& Savchenko 2012. PRINS OSCARS LAND: This publication. BARENTSøYA: Philippi 1973, Konstantinova \& Savchenko 2018. EDGEØYA: Philippi 1973. SPITSBERGEN: Lindberg 1866 as Jungermannia bicuspidata A $\beta *$ gracillima [Isfjorden], Arnell \& Mårtensson 1959 [W. Spitsbergen]. HAAKON VII LAND: This publication. HEER LAND: Philippi 1973. NoRdenskiöld LAND: Eurola 1968, Hadač 1989, Konstantinova \& Savchenko 2007, 2008b, Thingsgaard \& Damsholt 2007. OSCAR II LAND: Brattbakk et al. 1978, Kobayashi et al. 1990. PRINS_KARLS_ForLAND: Ellis et al. 2019c, Konstantinova \& Savchenko 2020a. SABINE LAND: Philippi 1973. WEedel__arLSBERG LAND: Rejment-Grochowska 1967, Karczmarz \& Święs 1988, 1989, Święs \& Karczmarz 1991a, Frisvoll \& Elvebakk 1996. NOTE: We are not aware of any primary report from Bjørnøya unless the report of Cephalozia bicuspidata by Watson (1922) can be referred there.

*** Cephalozia bicuspidata (L.) Dumort. var. bicuspidata [Jungermannia bicuspidata L., Eucephalozia bicuspidata (L.) Schiffn.]. SvalbarD: Berggren 1875a, Schiffner 1898, Frye \& Clark 1945, Le Gallo 1951, Smith 1990, Söderström 1995, Damsholt 2002, Söderström et al. 2002a, 2002b, Vilnet et al. 2012a, Hodgetts, Feldberg et al. 2016, Hodgetts \& Lockhart 2020. BJøRnøYa: Schiffner 1898, Watson 1922, Summerhayes \& Elton 1923a, Söderström 1995, Söderström et al. 2002b [rejecting earlier reports]. GuSTAV V LAND: Konstantinova et al. 2020a. PRINS OSCARS LAND: Konstantinova \& Savchenko 2008a, Newsham \& Goodall-Copestake 2021. BARENTSØYA: Konstantinova \& Savchenko 2018. SPITSBERGEN: Arnell \& Mårtensson 1959 [rejecting report by Lindberg 1867 (Isfjorden) and Arnell 1900 as Cephalozia ambigua (Prins Karls Forland)]. AlbBert I LAND: Gottsche et al. 1847. NoRdenSKiöld LAND: Hadač 1946, Hadač 1989, Konstantinova \& Savchenko 2007, Konstantinova \& Savchenko 2008a, Konstantinova \& Savchenko 2008b, Stebel et al. 2018. OSCAR II LAND: Boińska \& Gugnacka-Fiedor 1983. PRINS KaRlS Forland: Arnell 1900 [rejected as Cephalozia ambigua by "may represent ssp. arctica" fide Schuster 1969], 
Watson 1922, Summerhayes \& EIton 1923b. SøRKAPP LAND: Dubiel \& Olech 1990. WEEDEL_JARLSBERG_LAND: RejmentGrochowska 1967, Karczmarz \& Święs 1989, 1990a, Święs \& Karczmarz 1991a, 1991b, Frisvoll \& Elvebakk 1996 [with doubt], Frisvoll \& Blom 1997. NOTE: Cephalozia bicuspidata has often been reported s.lat. (i.e. including Cephalozia ambigua) and several reports have been, or should be, rejected as the latter species.

Cephaloziella (Spruce) Schiffn.

*** Cephaloziella arctogena (R.M.Schust.) Konstant. $\underline{\text { Sval- }}$ BARD: Söderström et al. 2007, Hodgetts 2015, Hodgetts \& Lockhart 2020, Konstantinova \& Savchenko 2020b. ㅂAAKON VII LAND: Thingsgaard \& Damsholt 2007.

*** Cephaloziella integerrima (Lindb.) Warnst. [Dichiton integerrimum (Lindb.) H.Buch]. SvalbarD: Düll 1983, Smith 1990, Söderström 1995 [rejecting earlier reports], Damsholt 2002 [with a "?"], Söderström et al. 2002a [rejecting earlier reports], Söderström et al. 2002b [rejecting earlier reports], Damsholt 2013, Hodgetts 2015, Hodgetts \& Lockhart 2020. NORDENSKIÖLD LAND: Konstantinova \& Savchenko 2007, 2008a, 2008b, 2020b. OSCAR II LAND: Arnell \& Mårtensson 1959, Frisvoll \& Elvebakk 1996 [rejecting earlier reports], Konstantinova \& Savchenko 2020b. SøRKAPP LAND: This publication. WEDEL JARLSBERG LAND: This publication. *** Cephaloziella polystratosa (R.M.Schust. et Damsh.) Konstant. SvalbarD: Hodgetts 2015, Hodgetts \& Lockhart 2020, Hodgetts et al. 2020. NoRdAustLANDET: Konstantinova \& Savchenko 2020b. GuSTAV V LAND: Konstantinova \& Savchenko 2012, 2015.

*** Cephaloziella uncinata R.M.Schust. SVALBARD: Schuster 1980, Düll 1983, Davison 1993, Söderström 1995, Grolle \& Long 2000, Konstantinova 2000, Söderström et al. 2002a, 2002b, Prey et al. 2014, Hodgetts 2015, Hodgetts \& Lockhart 2020. PRINS_OSCARS LAND: Konstantinova \& Savchenko 2008a. AlBERT I LAND: Thingsgaard \& Damsholt 2007, Konstantinova \& Savchenko 2008a. DICKSON_LAND: This publication, Frisvoll \& Elvebakk 1996. HAAKON VII LAND: This publication. NORDENSKIÖLD LAND: Hadač 1946 as Cephalozia striatula [cf. Frisvoll \& Elvebakk 1996], Frisvoll \& Elvebakk 1996, Damsholt 2002, Konstantinova \& Savchenko 2008b, Damsholt 2013. OSCAR II LAND: Arnell \& Mårtensson 1959 as Cephaloziella subdentata [cf. Frisvoll \& Elvebakk 1996]. SABINE LAND: Crundwell 1978, Frisvoll \& Elvebakk 1996. WEDEL_JARLSBERG_LAND: Frisvoll \& Elvebakk 1996, Schuster \& Konstantinova 1996.

*** Cephaloziella varians (Gottsche) Steph. S Svalbard: Bryhn 1906 as Cephalozia divaricata var. incurva, Steere \& Inoue 1978 as Cephaloziella arctica, Schuster 1980 as Cephaloziella arctica, Düll 1983 as Cephaloziella arctica, Söderström 1995 as Cephaloziella arctica, Damsholt 2002, Söderström et al. 2002a, 2002b, Ellis et al. 2011, 2015a, Damsholt 2013, Hodgetts 2015, Mamontov \& Vilnet 2017, Hodgetts \& Lockhart 2020. BJøRNØYA: Berggren 1875a as Jungermannia divaricata var. incurva, Söderström 1995 as Cephaloziella arctica, Frisvoll \& Elvebakk 1996 as Cephaloziella arctica, Damsholt 2002, Söderström et al. 2002b. GuSTAV V LAND: TYPE of Jungermannia divaricata var. incurva, Berggren 1875a, 1875 both as Jungermannia divaricata var. incurva, Geissler \& Bischler 1987 as Jungermannia divaricata var. incurva, Konstantinova \& Savchenko 2011, 2012. PRINS OSCARS LAND: Konstantinova \& Savchenko 2008a, Konstantinova et al. 2014, 2020b. $\underline{B} A R-$
ENTSøya: Philippi 1973 as Cephaloziella arctica, Konstantinova \& Savchenko 2018. EDGEØYA: Berggren 1875a as Jungermannia divaricata var. incurva, Philippi 1973 as Cephaloziella arctica, Heinemeijer 1979 as Cephaloziella arctica, Heinemeijer \& van Dijk 2004 as Cephaloziella arctica. SPITSBERgen: Persson 1942 as Cephaloziella alpina [Van Meijens Bay], Arnell \& Mårtensson 1959 as Cephaloziella arctica [W. Spitsbergen]. ALBERT_L LAND: SYNTYPE of Jungermannia divaricata var. incurva, Lindberg 1866 as Jungermannia divaricata var. incurva, Berggren 1875a as Jungermannia divaricata var. incurva, Geissler \& Bischler 1987 as Jungermannia divaricata var. incurva, Frisvoll \& Elvebakk 1996 as Cephaloziella arctica. DiCKSON LAND: Konstantinova \& Savchenko 2008c, 2011. HAAKON VII LAND: Konstantinova \& Savchenko 2006, Newsham \& Goodall-Copestake 2021. HEER_LAND: This publication. JAMES LAND: This publication. NATHORST LAND: This publication. NORDENSKIÖLD LAND: Eurola 1968 as Cephaloziella arctica, Hadač 1989 as Cephaloziella arctica, Konstantinova \& Savchenko 2007, 2008a, 2008b, Konstantinova et al. 2020b. NY -FrIESLAND: SYNTYPE of Jungermannia divaricata var. incurva, Lindberg 1866 as Jungermannia divaricata var. incurva, Geissler \& Bischler 1987 as Jungermannia divaricata var. incurva. OSCAR II LAND: Kobayashi et al. 1990 as Cephaloziella arctica, Newsham \& GoodallCopestake 2021. PRINS KARLS ForLAND: Konstantinova \& Savchenko 2020a. SABINE LAND: Philippi 1973 as Cephaloziella arctica. S. $ø$ RKAPP LAND: This publication. TORELL LAND: Rejment-Grochowska 1967 as Cephaloziella arctica. WEDEL JARLSBERG_LAND: Rejment-Grochowska 1967 as Cephaloziella arctica, Eurola 1968 as Cephaloziella arctica, Karczmarz \& Święs 1988, 1989, 1990a all as Cephaloziella arctica.

Clevea Lindb.

*** Clevea hyalina (Sommerf.) Lindb. [Marchantia hyalina Sommerf., Sauteria hyalina (Sommerf.) Lindb., Athalamia hyalina (Sommerf.) S.Hatt.]. Svalbard: Berggren 1875b, Kaalaas 1893a, Stephani 1898, Bryhn 1906, Herzog 1926, Arnell 1928, Jørgensen 1934, Müller 1952, Steere 1954, Arnell 1956, Worley 1972, Bonner 1977 as Clevea hyalina f. rufescens, Düll 1983, Schuster 1992, Söderström 1995 , Damsholt 2002, Söderström et al. 2002a, 2002b, Long et al. 2003, Rubasinghe 2011, Damsholt 2013, Hodgetts 2015, Hodgetts \& Lockhart 2020. BJøRNøYA: Sommerfeldt 1833, Berggren 1875a, Berggren 1875b, Frisvoll \& Elvebakk 1996. GuSTAV V LAND: Berggren 1875a, Konstantinova \& Savchenko 2012 as Clevea hyalina var. rufescens. B ENTSØYA: Konstantinova \& Savchenko 2018. SPITSBERGEN: Lindberg 1866 [Kingsbay]. DICKSON LAND: Frisvoll \& Elvebakk 1996 as Athalamia hyalina, Belkina et al. 2015. HAAKON VII LAND: Arnell \& Mårtensson 1959 as Clevea hyali$n a$ f. rufescens. JAMES LAND: Frisvoll 1981, Frisvoll \& Elvebakk 1996. NORDENSKIÖLD LAND: This publication. NYFRIESLAND: Berggren 1875a [rejected as Sauteria alpina by Lindberg 1877]. OSCAR II LAND: Arnell \& Mårtensson 1959 as Clevea hyalina f. rufescens. PRINS KARLS ForLAND: Konstantinova \& Savchenko 2020a. SøRKAPP LAND: Dubiel \& Olech 1990, Frisvoll \& Elvebakk 1996. WEDEL_ JARLSBERG LAND: This publication.

Cryptocolea R.M.Schust.

*** Cryptocolea imbricata R.M.Schust. SvalBarD: Düll 1983, Schuster 1983, Davison 1993, Söderström 1995, 
Schuster \& Konstantinova 1996, Grolle \& Long 2000, Konstantinova 2000, Damsholt 2002, Söderström et al. 2002a, 2002b, Damsholt 2013, Hodgetts 2015, Hodgetts \& Lockhart 2020. ANDRÉE LAND: Frisvoll \& Blom 1992, 1997, Konstantinova \& Savchenko 2020b. DickSON LAND: Frisvoll 1981, Frisvoll \& Elvebakk 1996. O-SCAR II LAND: Ellis et al. 2021. Diplophyllum (Dumort.) Dumort.

*** Diplophyllum albicans (L.) Dumort. [Jungermannia albicans L.]. Svalbard: Berggren 1875b, Müller 1914b, Buch 1928, Frye \& Clark 1946, Schuster \& Damsholt 1974, Düll 1983, Miller et al. 1983 [with a '?'], Smith 1990, Söderström 1995, Bisang et al. 1998, Engel \& Smith Merrill 1998, Paton 1999, Damsholt 2002, Söderström et al. 2002a, 2002b, Konstantinova 2003, Vilnet et al. 2010, Heinrichs et al. 2012, Damsholt 2013, Hodgetts 2015, Bakalin \& Vilnet 2018, Bakalin et al. 2020a, Hodgetts \& Lockhart 2020. BJøRnøYA: Summerhayes \& Elton 1923a, Söderström 1995, Frisvoll \& Elvebakk 1996, Frisvoll \& Blom 1997, Damsholt 2002, Söderström et al. 2002b. PRINS OsCARS LAND: Konstantinova \& Savchenko 2008a, 2015. ALBERT I LAND: Berggren 1875a, Schuster 1974, Frisvoll \& Elvebakk 1996, Frisvoll \& Blom 1997, Konstantinova \& Savchenko 2008a. NORDENSKIÖLD LAND: Konstantinova \& Savchenko 2008a. *** Diplophyllum taxifolium (Wahlenb.) Dumort. [Jungermannia taxifolia Wahlenb.]. SvalBARD: Müller 1914b, Buch 1928, Jørgensen 1934, Frye \& Clark 1946, Müller 1956a, Schuster 1974, Schuster \& Damsholt 1974, Düll 1983 as Diplophyllum taxifolium var. macrostictum [with a '?'], Smith 1990, Söderström 1995, Frisvoll \& Elvebakk 1996, Engel \& Smith Merrill 1998, Paton 1999, Damsholt 2002, Söderström et al. 2002a, 2002b, Hodgetts 2015, Hodgetts \& Lockhart 2020. PRINS OSCARS LAND: This publication. ALBERT I LAND: Berggren 1875a. HAAKON VII LAND: Arnell \& Mårtensson 1959 as Diplophyllum taxifolium var. macrostictum. NOTE: Except for the new report here, Diplophyllum taxifolium is only known from old sources.

Fuscocephaloziopsis Fulford

*** Fuscocephaloziopsis albescens (Hook.) Váňa et L.Söderstr. [Pleuroclada albescens (Hook.) Spruce, Pleurocladula albescens (Hook.) Grolle]. Svalbard: Schofield \& Crum 1972, Smith 1990, Söderström 1995, Paton 1999, Damsholt 2002, Söderström et al. 2002b, Vilnet et al. 2012a, Damsholt 2013, Feldberg et al. 2016. BJøRnøYa: Jørgensen 1934, Söderström 1995, Frisvoll \& Elvebakk 1996, Damsholt 2002, Söderström et al. 2002b. PRINS OSCARS LAND: This publication. EDGEØYA: Heinemeijer 1979, Frisvoll \& Elvebakk 1996. Spitsbergen: Philippi 1973 [Kvalvågen], Frisvoll \& Elvebakk 1996 [Kvalvågen, Billefjorden]. ALBERT_I LAND: Arnell \& Mårtensson 1959 ["seems to be represented mainly by its var. islandica"], Frisvoll \& Elvebakk 1996. DiCKSON LAND: This publication. HAAKON VII LAND: Arnell \& Mårtensson 1959 ['seems to be represented mainly by its var. islandica"]. NORDENSKIÖLD LAND: Arnell \& Mårtensson 1959 ["seems to be represented mainly by its var. islandica"], Frisvoll \& Elvebakk 1996, Konstantinova \& Savchenko 2008b, Prestø et al. 2014. OSCAR II LAND: Arnell \& Mårtensson 1959 ['seems to be represented mainly by its var. islandica']. PRINS_KARLS_ForLAND: Frisvoll \& Elvebakk 1996 [s.lat.]. NOTE: All reports of Fuscocephaloziopsis albescens should probably be referred to var. islandica if the variety should be recognized at all. Frisvoll \& Elvebakk (1996) rejected var. albescens completely from Svalbard.
* - var. albescens [f. albescens]. Svalbard: Schuster 1974, Düll 1983 [with a '?'], Hodgetts 2015 [with a '?']. * - var. islandica (Nees) Váňa et L.Söderstr. [Jungermannia islandica Nees, Pleurocladula islandica (Nees) Grolle, Pleurocladula albescens var. islandica (Nees) L.Söderstr. et Váňa]. S VALBARD: Berggren 1875b, Düll 1983, Söderström 1995, Söderström et al. 2002a, Hodgetts 2015, Hodgetts \& Lockhart 2020. BJøRnøYA: Berggren 1875a. ALBERT I LAND: Berggren 1875a. NORDENSKIÖLD LAND: Berggren 1875a. PRINS KARLS FoRLAND: Berggren 1875a.

*** Fuscocephaloziopsis lunulifolia (Dumort.) Váňa et L.Söderstr. [Cephalozia lunulifolia (Dumort.) Dumort.]. SVALBARD: Söderström 1995, Damsholt 2002, Söderström et al. 2002a, 2002b, Konstantinova 2003, Damsholt 2013, Bakalin \& Vilnet 2014, Hodgetts 2015, Hodgetts \& Lockhart 2020. WEDEL_JARLSBERG_LAND: Rejment-Grochowska 1967 as Cephalozia media, Karczmarz \& Święs 1989 as Cephalozia media, Frisvoll \& Blom 1992, Frisvoll \& Elvebakk 1996, Frisvoll \& Blom 1997.

*** Fuscocephaloziopsis pleniceps (Austin) Váňa et L.Söderstr. var. pleniceps [Cephalozia pleniceps (Austin) Lindb.]. SVVALBARD: Smith 1990, Söderström 1995, Damsholt 2002, 2013, Söderström et al. 2002a, 2002b, Vilnet et al. 2012a, Hodgetts 2015, Feldberg et al. 2016, Hodgetts \& Lockhart 2020. PrIns OSCARS LAND: This publication. SPITSBERGEN: Persson 1942 [Kingsbay]. Dickson LAND: Konstantinova \& Savchenko 2008c. HAAKON VII LAND: Konstantinova \& Savchenko 2006, 2008a. JAMES LAND: This publication, Frisvoll \& Elvebakk 1996. NORDENSKIÖLD LAND: Hadač 1946, Arnell \& Mårtensson 1959, Frisvoll \& Elvebakk 1996, Konstantinova \& Savchenko 2007, 2008a, 2008b. OSCAR II LAND: Arnell \& Mårtensson 1959, Kobayashi et al. 1990, Frisvoll \& Elvebakk 1996. PRINS KARLS FORLAND: Konstantinova \& Savchenko 2020a. S ABINE LAND: Crundwell 1978, Frisvoll \& Elvebakk 1996. WEDEL JARLSBERG LAND: Eurola 1968.

Gymnocolea (Dumort.) Dumort.

*** Gymnocolea inflata (Huds.) Dumort. subsp. inflata [Jungermannia inflata Huds.]. SvalBARD: Düll 1983, Geissler \& Bischler 1987 as Jungermannia inflata var. rigidiuscula, Söderström 1995, Paton 1999, Damsholt 2002, 2013, Söderström et al. 2002a, 2002b, Vilnet et al. 2010, 2012a, Shaw et al. 2015, Hodgetts 2015, Mamontov \& Vilnet 2017, Hodgetts \& Lockhart 2020. PRINS OSSCARS LAND: This publication, Konstantinova \& Savchenko 2015. EDGEØYA: Heinemeijer 1979, Frisvoll \& Elvebakk 1996, Heinemeijer \& van Dijk 2004. SPITSBERGEN: Berggren 1875b. NORDENSKIÖLD LAND: TYPE of Jungermannia inflata var. rigidiuscula, Berggren 1875a as Jungermannia inflata var. rigidiuscula, Bonner 1976 as Jungermannia inflata var. rigidiuscula, Bednarek-Ochyra et al. 1987c, Hadač 1989, Frisvoll \& Elvebakk 1996, Konstantinova \& Savchenko 2008a, 2008b, Konstantinova et al. 2020b. OSCAR II LAND: Inoue 1988, Kobayashi et al. 1990, Frisvoll \& Elvebakk 1996, Newsham \& Goodall-Copestake 2021. SABINE LAND: Philippi 1973, Frisvoll \& Elvebakk 1996. WEDEL_JARLSBERG_LAND: Rejment-Grochowska 1967, Karczmarz \& Święs 1989, Frisvoll \& Elvebakk 1996.

Gymnomitrion Corda

*** Gymnomitrion concinnatum (Lightf.) Corda [Cesius concinnatus (Lightf.) Gray 'Cesia concinnata']. SvalBard: Berggren 1875b, Müller 1909a, Herzog 1926, Arnell 1928, 
Harmsen \& Seidenfaden 1932, Jørgensen 1934, Frye \& Clark 1943, Steere 1954, Arnell 1956, Müller 1956a, Worley 1972, Schuster 1974, Schljakov 1981, Smith 1990, Söderström 1995, Frisvoll \& Elvebakk 1996, Paton 1999, Damsholt 2002, Söderström et al. 2002a, 2002b, Hodgetts 2015, Shaw et al. 2015, Patzak et al. 2016a, Hodgetts \& Lockhart 2020. BJøRNøYA: Berggren 1875a, Söderström 1995, Frisvoll \& Elvebakk 1996, Damsholt 2002, Söderström et al. 2002 b. GuSTAV V LAND: Lindberg 1866, Berggren 1875a. PRINS OSCARS LAND: Konstantinova \& Savchenko 2008a. B BARENTSøYA: Philippi 1973, Konstantinova \& Savchenko 2018. EDgeøya: Philippi 1973. Albert I Land: Lindberg 1866, Berggren 1875a, Arnell \& Mårtensson 1959, Thingsgaard \& Damsholt 2007, Konstantinova \& Savchenko 2008a. DICKSON LAND: This publication, Belkina et al. 2015. HAAKON_VII LAND: Arnell \& Mårtensson 1959. NORDENSKIÖLD LAND: Berggren 1875a, Hagen 1952, Eurola 1968, Hadač 1989, Konstantinova \& Savchenko 2007, 2008a, 2008b, Virtanen et al. 2013. OSCAR II LAND: Arnell \& Mårtensson 1959. PRINS KARLS FORLAND: Berggren 1875a, Konstantinova \& Savchenko 2020a. WEDEL JARLSBERG LAND: RejmentGrochowska 1967, Karczmarz \& Święs 1988, 1990b, Święs \& Karczmarz 1991a, 1991b, 1993a.

*** Gymnomitrion corallioides Nees [Cesius corallioides (Nees) Carruth. 'Cesia corallioides']. Svalbard: Berggren 1875b, Bryhn 1906, Müller 1909a, Jørgensen 1934, Frye \& Clark 1943, Steere 1954, Schuster \& Damsholt 1974, Schuster 1974, Düll 1983, Smith 1990, Söderström 1995, Frisvoll \& Elvebakk 1996, Paton 1999, Damsholt 2002, Söderström et al. 2002a, Hentschel et al. 2006, 2007, Heinrichs et al. 2007, Vilnet et al. 2010, Damsholt 2013, Hodgetts 2015, Shaw et al. 2015, Mamontov et al. 2018, Mamontov et al. 2019, Hodgetts \& Lockhart 2020. BJøRNØYA: Söderström 1995, Frisvoll \& Elvebakk 1996, Damsholt 2002. GuSTAV V LAND: Berggren 1875a. PRINS OSCARS LAND: Konstantinova \& Savchenko 2008a. B ARENTSØYA: Hoffman 1968, Philippi 1973, Konstantinova \& Savchenko 2018. EDGEØYa: Philippi 1973. KONG_KarLS_LAND: Arnell 1900, Hoffman 1968. SPITSBERGEN: Arnell \& Mårtensson 1959 [W. Spitsbergen], Vilnet et al. 2011. ALbert I LAND: Berggren 1875a, Frisvoll 1978. DICKSON LAND: This publication. HEER LAND: Philippi 1973. NNORDENSKIÖLD_LAND: Hadač 1946, Hagen 1952, Eurola 1968, Hadač 1989, Konstantinova \& Savchenko 2007, 2008a, 2008b, Thingsgaard \& Damsholt 2007, Virtanen et al. 2013, Konstantinova et al. 2020b. NYY-FrIESLAND: This publication. OSCAR II LAND: This publication. PrINS KARLS FoRLAND: Konstantinova \& Savchenko 2020a. SABINE LAND: Philippi 1973. S- ØֵKAPP LAND: Dubiel \& Olech 1990, Wietrzyk et al. 2016. WEDEL JARLSBERG LAND: Rejment-Grochowska 1967, Eurola 1968, BednarekOchyra et al. 1987b, Karczmarz \& Święs 1990a. NOTE: We are not aware of any primary report from Bjørnøya.

* - var. minutum Lindb. Albert I LAND: TYPE, Lindberg 1866. HAAKON_VII LAND: Lindberg 1866. NOTE: The variety is not recognized in recent treatments, and we do not think it is worth to recognize either. However, we do not know if this name refers to Gymnomitrion coralloides or to any other species as we have not seen any type material.

Haplomitrium Nees

*** Haplomitrium hookeri (Lyell ex Sm.) Nees. Svalbard: Grolle 1964, Schuster 1966, 1967, Worley 1969, Schofield \& Crum 1972, Schuster \& Damsholt 1974, Schljakov 1976,
Godfrey 1977, Engel 1981, Düll 1983, Schuster 1983, Smith 1990, Bartholomew-Began 1991, Parihar et al. 1994, Söderström 1995, Schuster \& Konstantinova 1996, Paton 1999, Schumacker et al. 1999, Bapna \& Kachroo 2000a, Konstantinova 2000, 2003, Damsholt 2002, Söderström et al. 2002a, 2002b, Sotiaux et al. 2006, Engel \& Glenny 2008, Damsholt 2013, Duckett \& Slack 2013, Hodgetts 2015, Hodgetts \& Lockhart 2020. NNORDENSKIÖLD LAND: Arnell \& Mårtensson 1959, Frisvoll \& Blom 1992, Frisvoll \& Elvebakk 1996, Frisvoll \& Blom 1997.

Isopaches H.Buch

*** Isopaches bicrenatus (Schmidel ex Hoffm.) H.Buch [Lophozia bicrenata (Schmidel ex Hoffm.) Dumort.]. SvaLBARD: Frye \& Clark 1945, Schuster 1969 ['not confirmed'], Düll 1983, Söderström 1995, Paton 1999, Damsholt 2002, 2013, Söderström et al. 2002a, 2002b, Hodgetts 2015, Hodgetts \& Lockhart 2020. BüNSOW LAND: Summerhayes \& Elton 1923a, Frisvoll \& Blom 1997. NORDENSKIÖLD LAND: Watson 1922, Summerhayes \& Elton 1923b, Konstantinova \& Savchenko 2008b. WEDEL J Grochowska 1967, Frisvoll \& Blom 1992, 1997, Frisvoll \& Elvebakk 1996.

Jungermannia L.

*** Jungermannia polaris Lindb. [Jungermannia pumila * polaris (Lindb.) Berggr., Aplozia polaris (Lindb.) Bryhn et Kaal. 'Haplozia', Jungermannia pumila subsp. polaris (Lindb.) R.M.Schust., Solenostoma polare (Lindb.) R.M.Schust.]. SvalBarD: Berggren 1875b, Bryhn 1906, , Herzog 1926, Arnell 1928, Schuster et al. 1959, Düll 1983, Smith 1990, Söderström 1995, Paton 1999, Söderström et al. 2002a, Söderström et al. 2002b, Hodgetts 2015, Konstantinova \& Vilnet 2016, Hodgetts \& Lockhart 2020. BJøRNØYA: Berggren 1875a, Váňa 1973, Söderström 1995, Frisvoll \& Elvebakk 1996, Damsholt 2002, Söderström et al. 2002b. GustaV V LAND: Konstantinova \& Savchenko 2012. PRINS_OSCARS_LAND: This publication. BARENTSøYA: Konstantinova \& Savchenko 2018. DICKSON LAND: Frisvoll 1981, Konstantinova \& Savchenko 2008c, 2011. KON VII LAND: Lindberg 1866, Arnell \& Mårtensson 1959, Arnell \& Mårtensson 1959 as Jungermannia schiffneri, Váňa 1973, Grolle 1976, Geissler \& Bischler 1987, Konstantinova \& Savchenko 2006. HEER LAND: This publication. JAMES LAND: This publication. NATHORST LAND: This publication. NNORDENSKIÖLD LAND: Berggren 1875a, Konstantinova \& Savchenko 2007, 2008a, 2008b. NY-FRIESLAND: TYPE, Schuster 1969, Frisvoll \& Elvebakk 1996, Damsholt 2002, 2013. OSCAR II LAND: Arnell \& Mårtensson 1959. PRINS KaRLS_FORLAND: Konstantinova \& Savchenko 2020a. SØRKAPP LAND: This publication. WEDEL JARLSBERG LAND: This publication.

*** Jungermannia pumila With. [Solenostoma pumilum (With.) Müll.Frib.]. SVALBARD: Schuster 1969, Worley 1972, Váňa 1973, Söderström 1995, Damsholt 2002 [with a ?], Konstantinova 2003, Damsholt 2013, Schäfer-Verwimp et al. 2019. BృøRnøYA: Berggren 1875a, Söderström 1995. DICKSON_LAND: This publication. HAAKON_VII LAND: Arnell \& Mårtensson 1959. NATHORST LAND: This publication. OSCAR II LAND: Arnell \& Mårtensson 1959. PRINS KARLS FORLAND: Konstantinova \& Savchenko 2020a. SøRKAPP LAND: This publication. WEDEL JARLSBERG LAND: This publication. NOTE: Many identifications are s.lat. or only preliminary and may belong to Jungermannia polaris. 
Lophozia (Dumort.) Dumort.

** Lophozia fuscovirens Bakalin et Vilnet. SVALBARD: Hodgetts \& Lockhart 2020, Hodgetts et al. 2020, Konstantinova et al. 2020a. HEER LAND: This publication. PRINS KARLS Forland: Ellis et al. 2019c, Konstantinova \& Savchenko 2020a.

** Lophozia murmanica Kaal. SvalBard: Herzog 1926 as Sphenolobus groenlandicus, Steere 1954 as Lophozia groenlandica, Arnell 1956 as Lophozia groenlandica, Schuster 1961 as Lophozia groenlandica, Schljakov 1980 as Lophozia groenlandica, Düll 1983 as Lophozia groenlandica, Damsholt 2002, Konstantinova 2003 as Lophozia wenzelii var. groenlandica, Söderström et al. 2007 as Lophozia groenlandica, Kalinauskaitë \& von Cräutlein 2010, Hodgetts 2015 as Lophozia groenlandica, Hodgetts \& Lockhart 2020, Konstantinova \& Savchenko 2020b. GuSTAV V LAND: Konstantinova \& Savchenko 2012 as Lophozia wenzelii var. groenlandica. B B ARENTSØYA: Konstantinova \& Savchenko 2018. SPITSBERGEN: Bakalin 2004 as Lophozia wenzelii var. groenlandica [Kongsfjorden], Bakalin 2005 as Lophozia wenzelii var. groenlandica [Kings Bay]. ALBERT I LAND: Thingsgaard \& Damsholt 2007. NORDENSKIÖLD LAND: Bryhn 1909 as Lophozia groenlandica, Müller 1910a as Sphenolobus groenlandicus, Konstantinova \& Savchenko 2008a as Lophozia wenzelii var. groenlandica, Konstantinova \& Savchenko $\mathbf{2 0 0 8 b}$ as Lophozia wenzelii var. groenlandica. PRINS KARLS FORLAND: Konstantinova \& Savchenko 2020a. WEDEL JARLSBERG_LAND: Rejment-Grochowska 1967 as Lophozia groenlandica, Karczmarz \& Święs 1988 as Lophozia groenlandica, Karczmarz \& Święs 1989, 1990b both as Lophozia groenlandica, Święs \& Karczmarz 1991a, 1991b, 1993b all as Lophozia groenlandica. NOTE: The nomenclature in Lophozia is confusing, and the same name has been used for different species (see Söderström et al., 2013). The name Lophozia groenlandica is used for at least four Lophozia taxa, L. murmanica, L. wenzelii, L. schusterana and L. ventricosa. We think that many of the Svalbard records can be referred to our Lophozia murmanica concept.

** Lophozia savicziae Schljakov. Svalbard: Konstantinova 2000, Söderström et al. 2002a, Bakalin 2005 [Storfjord], Hodgetts 2015, Hodgetts \& Lockhart 2020, Konstantinova \& Savchenko 2020b. Olav V Land: This publication.

*** Lophozia silvicola H.Buch [Lophozia ventricosa var. silvicola (H.Buch) E.W.Jones]. SvalBARD: Söderström et al. 2007, Ellis et al. 2014a, Hodgetts 2015, Hodgetts \& Lockhart 2020. BARENTSøya: Konstantinova \& Savchenko 2018. ALBERT I LAND: Thingsgaard \& Damsholt 2007.

*** Lophozia silvicoloides N.Kitag. SvalbarD: Vilnet et al. 2005 as Lophozia silvicoloides var. paroica, Söderström et al. 2007, Vilnet et al. 2010, Bakalin 2011, Fedosov et al. 2015, Hodgetts 2015, Bakalin \& Vilnet 2019, Hodgetts \& Lockhart 2020, Hodgetts et al. 2020, Konstantinova \& Savchenko 2020b, Konstantinova et al. 2020a. NORDENSKIÖLD LAND: Konstantinova \& Savchenko 2007, 2008a, 2008b.

* Lophozia subapiculata R.M.Schust. et Damsh. SvaLBARD: Hodgetts \& Lockhart 2020, Hodgetts et al. 2020, Konstantinova \& Savchenko 2020b. BARENTSøYA: Konstantinova \& Savchenko 2018.

** Lophozia svalbardensis Konstant., Vilnet et Mamontov. GUSTAV V LAND: TYPE, Konstantinova et al. 2020a.

*** Lophozia ventricosa (Dicks.) Dumort. [Jungermannia ventricosa Dicks.]. Svalbard: Bryhn 1906, Harmsen \& Seidenfaden 1932, Frye \& Clark 1945, Söderström 1995, Paton 1999, Söderström et al. 2002a, 2002b, Hodgetts 2015, Hodgetts \& Lockhart 2020. B merhayes \& Elton 1923a, Söderström 1995, Frisvoll \& Elvebakk 1996, Söderström et al. 2002b. Frisvoll \& Elvebakk 1996. KONG KarLS LAND: Arnell 1900, Frisvoll \& Elvebakk 1996. AlBERT I LAND: Lindberg 1866 as Jungermannia ventricosa $\alpha$ * gemmipara, Jungermannia ventricosa $\mathrm{b}$ laxa and Jungermannia ventricosa $\beta *$ pulchella, Frisvoll \& Elvebakk 1996. NORDENSKIÖLD LAND: Stebel et al. 2018. OSCAR II LAND: Watson 1922, Summerhayes \& Elton 1923a, 1923b, Frisvoll \& Elvebakk 1996. Prins KARLS ForLAND: Arnell 1900, Watson 1922, Frisvoll \& Elvebakk 1996.

* - var. longiflora (Nees) Macoun [Lophozia longiflo$r a$ (Nees) Schiffn.]. S Svalbard: Frye \& Clark 1945. BuøønøøY: Watson 1922. NORDENSKIÖLD LAND: Konstantinova $\&$ Savchenko 2008b. PRINS KARLS FORLAND: Konstantinova \& Savchenko 2020a.

* - var. ventricosa. Svalbard: Damsholt 2002, Söderström et al. 2007, Damsholt 2013. Prins OsCARS LAND: This publication. ALBERT I LAND: Thingsgaard \& Damsholt 2007. * Lophozia wenzelii (Nees) Steph. [Jungermannia wenzelii Nees]. Svalbard: Bryhn 1906, Müller 1910b, Arnell 1928 as Jungermannia wenzelii, Jørgensen 1934, Frye \& Clark 1945, Müller 1954b, Worley 1972, Smith 1990, Söderström 1995, Paton 1999, Söderström et al. 2002a, 2002b, Damsholt 2013, Hodgetts 2015, Hodgetts \& Lockhart 2020. GuSTAV V LAND: This publication. PRINS OSCARS LAND: This publication. Barentsøya: Philippi 1973. EDGEØYa: Philippi 1973. Kong Karls Land: Arnell 1900. HAAKON VII LAND: Arnell \& Mårtensson 1959, Newsham \& Goodall-Copestake 2021. Heer Land: Philippi 1973. Nordenskiöld LAND: Arnell \& Mårtensson 1959, Hadač 1989, Frisvoll \& Elvebakk 1996. OSCAR II LAND: Arnell \& Mårtensson 1959, Kobayashi et al. 1990. WEDEL JARLSBERG_LAND: RejmentGrochowska 1967, Frisvoll \& Elvebakk 1996.

* - var. lapponica H.Buch et S.W.Arnell. S Bakalin 2004, 2005, Vilnet et al. 2005, 2010, Söderström et al. 2007, Damsholt 2013, Bakalin \& Vilnet 2019, Konstantinova et al. 2020a. BARENTSØYA: Konstantinova \& Savchenko 2018. ALBERT I LAND: Thingsgaard \& Damsholt 2007. NORDENSKIÖLD LAND: Konstantinova \& Koroleva 2003, Konstantinova \& Savchenko 2007, 2008b, Konstantinova et al. 2020b. PRINS KARLS FORLAND: Konstantinova \& Savchenko 2020a.

* - var. wenzelii. Svalbard: Arnell 1956, Schuster 1969, Schuster \& Damsholt 1974, Düll 1983, Damsholt 2002. NORDENSKIÖLD LAND: Konstantinova \& Savchenko 2008b.

Lophoziopsis Konstant. et Vilnet

*** Lophoziopsis excisa (Dicks.) Konstant. et Vilnet [Lophozia excisa (Dicks.) Dumort.]. SvalbarD: Düll 1983, Damsholt 2002 [with a "?"], Söderström et al. 2002a, Vilnet et al. 2005, Vilnet et al. 2010, 2012a, Damsholt 2013, Mamontov \& Vilnet 2017, Bakalin \& Vilnet 2019, Konstantinova et al. 2020a. ?미№YA: Söderström 1995. PRINS OSCARS LAND: This publication. AlBERT I LAND: Thingsgaard \& Damsholt 2007. HEER LAND: This publication. NORDENSKIÖLD LAND: Arnell \& Mårtensson 1959 as Lophozia kiaerii, Konstantinova \& Savchenko 2007, 2008a, 2008b. OSCAR II LAND: Arnell \& Mårtensson 1959 as Lophozia kiaerii, Newsham \& Goodall-Copestake 2021. $\underline{\text { } ø \text { RKAPP LAND: }}$ 
This publication. Wedel JarLSBERG LAND: Eurola 1968. NOTE: Frisvoll \& Elvebakk (1996) stated that the presence of Lophoziopsis excisa on Svalbard "needs confirmation". However, more recent works have shown that the species occurs on Svalbard, but often it is scattered among other bryophytes, never occurring in large mats and therefore, perhaps, overlooked. Only reported with doubt from Bjørnøya and Prins Karls Forland.

** — var. elegans (R.M.Schust.) Konstant. et Vilnet [Lophozia excisa var. elegans R.M.Schust.]. SVALBARD: Hodgetts 2015, Hodgetts \& Lockhart 2020. BARENTSøYA: Konstantinova \& Savchenko 2018. HAAKON_VII LAND: Konstantinova \& Savchenko 2006, 2008a.

** — var. excisa. SVALBARD: Schljakov 1980 as Lophozia major, Söderström et al. 2007, Hodgetts 2015, Hodgetts \& Lockhart 2020. ?BృøRnøYA: Watson 1922 as Lophozia excisa var. cylindracea with doubt. BARENTSøYA: Konstantinova \& Savchenko 2018. ?PRINS KARLS_FoRLAND: Watson 1922 as Lophozia excisa var. cylindracea with doubt.

** Lophoziopsis jurensis (Meyl. ex Müll.Frib.) Mamontov et Vilnet. SVALBARD: Schuster 1969 as Lophozia latifolia, Schuster \& Damsholt 1974 as Lophozia latifolia, Steere \& Inoue 1978 as Lophozia latifolia, Schljakov 1980 as Lophozia jurensis, Düll 1983 as Lophozia latifolia, Frisvoll \& Blom 1992 as Lophozia latifolia, Söderström 1995 as Lophozia latifolia, Frisvoll \& Elvebakk 1996 as Lophozia latifolia ["in need of confirmation"], Grolle \& Long 2000 as Lophozia latifolia, Damsholt 2002 as Lophozia latifolia, Söderström et al. 2002a as Lophozia latifolia, Söderström et al. 2002b as Lophozia latifolia, Damsholt 2013 as Lophozia latifolia, Hodgetts \& Lockhart 2020. GuSTAV V LAND: Konstantinova \& Savchenko 2012 as Lophoziopsis propagulifera. PRINS OSCARS LAND: This publication. BARENTSøYA: This publication. (ALBERT I LAND: Arnell \& Mårtensson 1959 as Lophozia latifolia [rejected by Grolle 1967]). (HAAKON VII LAND: Arnell \& Mårtensson 1959 as Lophozia latifolia [rejected by Grolle 1967]). (NoRdEnsKIÖLD LAND: Arnell \& Mårtensson 1959 as Lophozia latifolia [rejected by Grolle 1967], Eurola 1971 as Lophozia cf. latifolia). OSCAR II LAND: Arnell \& Mårtensson 1959 as Lophozia latifolia, Grolle 1967 as Lophozia latifolia, Frisvoll \& Blom 1997 as Lophozia latifolia. NOTE: All reports of Lophozia latifolia (=Lophoziopsis jurensis) from Svalbard, except one, were rejected by Grolle (1967), but later reports confirm its presence there.

*** Lophoziopsis longidens (Lindb.) Konstant. et Vilnet [Jungermannia longidens Lindb., Lophozia longidens (Lindb.) Macoun]. SvalBARD: Arnell 1928, Jørgensen 1934, Frye \& Clark 1945, Müller 1954b, Arnell 1956, Smith 1990, Söderström 1995, Paton 1999, Bapna \& Kachroo 2000b, Damsholt 2002, Söderström et al. 2002a, 2002b, Damsholt 2013. BJØRNØYA: Watson 1922, Summerhayes \& Elton 1923a, Söderström 1995, Frisvoll \& Elvebakk 1996 Damsholt 2002, Söderström et al. 2002b. KONG KARLS LAND: Arnell 1900, Müller 1910b, Frisvoll \& Elvebakk 1996. №RdENSKIÖLD LAND: Konstantinova \& Savchenko 2008b. OSCAR II LAND: Watson 1922, Summerhayes \& Elton 1923a, 1923b, Frisvoll \& Elvebakk 1996. WEDEL JARLSBERG LAND: Rejment-Grochowska 1967, Karczmarz \& Święs 1989, Frisvoll \& Elvebakk 1996.

** - subsp. longidens. Svalbard: Schuster 1969 [with doubt], Düll 1983 [with a '?'], Söderström et al. 2002b, Hodgetts 2015, Hodgetts \& Lockhart 2020. BJøRNøYA: Söderström et al. $2002 \mathrm{~b}$.
*** Lophoziopsis pellucida (R.M.Schust.) Konstant. et Vilnet [Lophozia pellucida R.M.Schust.]. SVVALBARD: Söderström 1995, Grolle \& Long 2000, Konstantinova 2000, Damsholt 2002, Söderström et al. 2002b, Hodgetts 2015, Konstantinova \& Savchenko 2020b. NoRDAustLANDET: Konstantinova \& Savchenko 2015. DICKSON LAND: This publication. HAAKON VII LAND: Frisvoll \& Blom 1992, 1997, Frisvoll \& Elvebakk 1996. №RDENSKIÖLD LAND: Frisvoll \& Blom 1997. Nㅡ는 FRIESLAND: This publication.

** — var. minor (R.M.Schust.) L.Söderstr. et Váňa [Lophozia pellucida var. minor R.M.Schust.]. SvALBARD: Söderström et al. 2002a, Söderström et al. 2007, Hodgetts \& Lockhart 2020. GuSTAV V LAND: Konstantinova \& Savchenko 2012. SPITSBERGEN: Bakalin 2005 [W Svalbard Isl.]. №RDENSKIÖLD LAND: Schuster \& Konstantinova 1996.

** — var. pellucida. S Svalbard: Söderström et al. 2002a, Söderström et al. 2007, Hodgetts \& Lockhart 2020. GuSTAV V LAND: Konstantinova \& Savchenko 2012. DICKSON LAND: Bakalin 2005.

*** Lophoziopsis polaris (R.M.Schust.) Konstant. et Vilnet [Lophozia polaris (R.M.Schust.) R.M.Schust. et Damsh.]. SVIAlBARD: Düll 1983 [with a '?'], Söderström 1995, Konstantinova 2000, Damsholt 2002, Söderström et al. 2002a, 2002b, Vilnet et al. 2010, Konstantinova et al. 2020a. GustaV V LAND: Konstantinova \& Savchenko 2012, 2015. PRINS OSCARS LAND: Konstantinova \& Savchenko 2015, 2020b. BARENTSøYA: Konstantinova et al. 2014, Konstantinova \& Savchenko 2018. DICKSON_LAND: This publication. HAAKON VII LAND: Konstantinova \& Savchenko 2006. HEER LAND: Konstantinova et al. 2020b. JAMES_LAND: This publication. NATHORST LAND: This publication. NORDENSKIÖLD LAND: Hadač 1989, Frisvoll \& Elvebakk 1996, Frisvoll \& Blom 1997, Konstantinova et al. 2020b. NyFRIESLAND: This publication. OSSCAR II LAND: Koroleva \& Borovichev 2014. PRINS KARLS FORLAND: Konstantinova \& Savchenko 2020a. $\underline{\text { S}} \emptyset \mathrm{RKAPP} L \underline{L}$ AND: This publication. WEDEL JARLSBERG LAND: This publication.

** — var. polaris. SVALBARD: Hodgetts 2015, Hodgetts \& Lockhart 2020. PRINS OSCARS LAND: Konstantinova \& Savchenko 2008a. HAAKON VII LAND: Konstantinova \& Savchenko 2008a. NORDENSKIÖLD LAND: Konstantinova \& Savchenko 2008a.

** — var. sphagnorum (R.M.Schust.) Konstant. et Vilnet [Lophozia polaris var. sphagnorum (R.M.Schust.) R.M.Schust. et Damsh.]. S Svalbard: Hodgetts 2015, Hodgetts \& Lockhart 2020. NNORDENSKIÖLD LAND: Konstantinova \& Savchenko 2007, 2008a, 2008b

** Lophoziopsis rubrigemma (R.M.Schust.) Konstant. et Vilnet [Lophozia rubrigemma R.M.Schust.]. SVALBARD: Konstantinova 2000, Söderström et al. 2002a, Hodgetts 2015, Hodgetts \& Lockhart 2020, Konstantinova \& Savchenko 2020b. GuSTAV V LAND: Konstantinova \& Savchenko 2012, 2015. ?BARENTSøYA: Konstantinova \& Savchenko 2018 as cf. rubrigemma. ?№RDENSKIÖLD_LAND: Konstantinova \& Savchenko 2008 b as cf. rubrigemma. NOTE: Lophoziopsis rubrigemma has only been reported with some doubt from Barentsøya and Nordenskiöld Land.

Mannia Corda

*** Mannia triandra (Scop.) Grolle. SVALbard: Borovichev et al. 2015, Hodgetts 2015 [with a '?'], Borovichev \& Bakalin 2016, Hodgetts \& Lockhart 2020, Konstantinova \& Savchenko 2020b. OSSCAR II LAND: Borovichev 2010. 
Marchantia L.

*** Marchantia polymorpha L. Svalbard: Kaalaas 1893a, Bryhn 1906, Müller 1907b, Jørgensen 1934, Müller 1952, Bischler 1984, Bischler-Causse 1989, Smith 1990, Söderström et al. 2002b, Konstantinova 2003. BıøRnøYA: Berggren 1875a, Söderström et al. 2002b. GuSTAV V LAND: Berggren 1875a, 1875b. KONG KaRLS LAND: Arnell 1900. SPITSBERGEN: Sommerfeldt 1833 [Vest-Spitsbergen], Polunin 1945 [Kongsfjorden]. Albert I LAND: Berggren 1875a. NORDENSKIÖLD LAND: Hadač 1946, 1989. WEDEL___ARLSBERG_LAND: Rejment-Grochowska 1967, Karczmarz \& Święs 1989, Święs \& Karczmarz 1991b.

*** — subsp. montivagans Bischl. et Boissel.-Dub. SvaLBARD: Düll 1983 as Marchantia alpestris, Söderström 1995 as Marchantia alpestris, Frisvoll \& Elvebakk 1996 as Marchantia alpestris, Paton 1999, Damsholt 2002 as Marchantia alpestris, Söderström et al. 2002a, Söderström et al. 2002b, Damsholt 2013 as Marchantia alpestris, Hodgetts 2015, Hodgetts \& Lockhart 2020. BJøRNøYA: Söderström 1995 as Marchantia alpestris, Frisvoll \& Elvebakk 1996 as Marchantia alpestris, Damsholt 2002 as Marchantia alpestris, Söderström et al. 2002b. GuSTAV V LAND: Lindberg 1866 as Marchantia polymorpha $\mathrm{B}$ alpestris, Konstantinova \& Savchenko 2012. BAREnTSøya: Philippi 1973 as Marchantia alpestris, Konstantinova \& Savchenko 2018. EDGEØYA: Philippi 1973 as Marchantia alpestris. AlBERT I LAND: Lindberg 1866 as Marchantia polymorpha B alpestris. DICKSON LAND: Belkina et al. 2015. HAAAKON_VII LAND: Arnell \& Mårtensson 1959 as Marchantia alpestris. JAMES LAND: This publication. NORDENSKIÖLD LAND: Arnell \& Mårtensson 1959 as Marchantia alpestris, Konstantinova \& Savchenko 2008b as Marchantia alpestris, Prestø et al. 2014. O- $\underline{S C A R}$ II LAND: Arnell \& Mårtensson 1959 as Marchantia alpestris, Prestø et al. 2014. PRINS_KARLS_FORLAND: Konstantinova \& Savchenko 2020a. WEDEL JARLSBERG LAND: RejmentGrochowska 1967 as Marchantia alpestris. NOTE: We are not aware of any primary report of subsp. montivagans from Bjørnøya and Dickson Land.

*** — subsp. ruderalis Bischl. et Boissel.-Dub. SVALBARD: Düll 1983 as , Söderström 1995 as Marchantia polymorpha, Frisvoll \& Elvebakk 1996 as Marchantia polymorpha, Damsholt 2002 as Marchantia latifolia, Söderström et al. 2002a, Söderström et al. 2002b, Hodgetts 2015, Ellis et al. 2019a, Hodgetts \& Lockhart 2020. DICKSON_LAND: This publication. Wedel Jarlsberg Land: Frisvoll \& Blom 1997 as Marchantia polymorpha. NOTE: Subsp. ruderalis has only been confirmed from anthropogenic areas and all other reports should probably be subsp. montivagans.

*** Marchantia quadrata Scop. [Preissia quadrata (Scop.) Nees, Chomiocarpon quadratum (Scop.) Lindb.]. SvalBARD: Schiffner 1893, Bryhn 1906 as Chomocarpon commutatus, Müller 1907b, Jørgensen 1934, Horikawa 1950 as Preissia quadrata, Düll 1983, Smith 1990, Söderström 1995, Frisvoll \& Elvebakk 1996, Paton 1999, Damsholt 2002, Söderström et al. 2002b, Long \& Crandall-Stotler 2020. BJøRNØYA: Watson 1922, Summerhayes \& Elton 1923a, Söderström 1995, Frisvoll \& Elvebakk 1996, Damsholt 2002, Söderström et al. 2002b. GuSTAV V LAND: Konstantinova \& Savchenko 2012. PRINS OSCARS LAND: This publication. BARENTSøYA: Philippi 1973, Konstantinova \& Savchenko 2018. DICKSON LAND: Belkina et al. 2015. HAAKON VII LAND: Arnell \& Mårtensson 1959, Frisvoll 1978. NATHORST LAND: This pub- lication. NORDENSKIÖLD LAND: This publication, Prestø et al. 2014. OSCAR II LAND: Arnell \& Mårtensson 1959. PRINS KARLS FORLAND: Konstantinova \& Savchenko 2020a. WEDEL JARLSBERG_LAND: Rejment-Grochowska 1967.

* - subsp. hyperborea (R.M.Schust.) Borovich. [Preissia quadrata subsp. hyperborea R.M.Schust.]. S VALBARD: Söderström et al. 2007, Hodgetts 2015, Hodgetts \& Lockhart 2020. B ARENTSØYA: Konstantinova \& Savchenko 2018. SPITSBERGEN: Thingsgaard \& Damsholt 2007. DICKSON LAND: This publication. HAAKON VII LAND: This publication. J JAMES LAND: This publication. OSCAR II LAND: This publication. PRINS KARLS ForLAND: This publication.

* - subsp. quadrata. SVALBARD: Schuster 1992, Söderström et al. 2002a, Damsholt 2013, Hodgetts 2015, Hodgetts $\&$ Lockhart 2020. NOTE: It is possible that only subsp. hyperborea occurs on Svalbard.

*** Marchantia romanica (Radian) D.G.Long, Crand.-Stotl., L.L.Forrest et J.C.Villarreal [Bucegia romanica Radian]. SVALBARD: Ellis et al. 2016a, Xiang et al. 2016, Sofronova \& Potemkin 2018, Hodgetts \& Lockhart 2020. NorDAustLANDET: Xiang et al. 2016. GuSTAV V LAND: Konstantinova et al. 2014. PRINS OSCARS LAND: Konstantinova et al. 2014. B BARENTSøYA: Konstantinova et al. 2014 Konstantinova \& Savchenko 2018. DICKSON LAND: Konstantinova et al. 2014. HAAKON VII LAND: This publication. OSCAR II LAND: This publication. $\underline{S}_{A B I N E}$ LAND: Konstantinova et al. 2014.

Marsupella Dumort.

*** Marsupella apiculata Schiffn. [Gymnomitrion apiculatum (Schiffn.) Müll.Frib.]. SVALBARD: Schuster \& Damsholt 1974, Schuster 1974, Godfrey \& Schofield 1979, Schljakov 1981, Düll 1983, Söderström 1995, Paton 1999, Damsholt 2002, Söderström et al. 2002a, 2002b, Konstantinova 2003, Vilnet et al. 2010, Hodgetts 2015, Mamontov et al. 2018, Mamontov et al. 2019, Hodgetts \& Lockhart 2020. PRINS OSCARS LAND: Konstantinova \& Savchenko 2008a, Konstantinova \& Savchenko 2015. AlbBert I LAND: Arnell \& Mårtensson 1959, Frisvoll \& Blom 1992, Frisvoll \& Elvebakk 1996, Frisvoll \& Blom 1997, Thingsgaard \& Damsholt 2007, Konstantinova \& Savchenko 2008a. HAAKON VII LAND: Frisvoll \& Blom 1992, Frisvoll \& Blom 1997. №RDENSKIÖLD LAND: Konstantinova \& Savchenko 2007, 2008a, 2008b, Konstantinova et al. 2020b. OSCAR II LAND: Kobayashi et al. 1990, Frisvoll \& Blom 1992, 1997, Frisvoll \& Elvebakk 1996.

*** Marsupella arctica (Berggr.) Bryhn et Kaal. [Sarcoscyphos emarginatus var. arcticus Berggr., Marsupella emarginata var. arctica (Berggr.) Frye et L.Clark]. SvaLBARD: Berggren 1875b, Bryhn 1906, Herzog 1926 as Marsupella groenlandica, Schuster 1950, Müller 1956a, Persson \& Gjaerevoll 1961, Schuster \& Damsholt 1974, Schljakov 1981, Düll 1983, Geissler \& Bischler 1985, Geissler \& Bischler 1985, Vitt et al. 1987, Long et al. 1990, Söderström 1995, Schuster \& Konstantinova 1996, Paton 1999, Konstantinova 2000, Söderström et al. 2002a, 2002b, Vilnet et al. 2010, Hodgetts 2015, Shaw et al. 2015, Mamontov et al. 2018, 2019, Hodgetts \& Lockhart 2020. NORDAUSTLANDET: Konstantinova \& Savchenko 2015, 2020b. PrINS OSCARS LAND: Konstantinova \& Savchenko 2008a, Konstantinova et al. 2020 b. SPITSBERGEN: Vilnet et al. 2011. ALBERT I LAND: TYPE, Berggren 1875a, Müller 1909a, Frye \& Clark 1943, Schuster 1974, Grolle 1976, Frisvoll \& Elvebakk 1996, Frisvoll \& Blom 1997, Damsholt 2002, 2013. N_ORDENSKIÖLD LAND: 
Frisvoll \& Elvebakk 1996, Frisvoll \& Blom 1997, Konstantinova \& Savchenko 2007, 2008a, 2008b, Prestø et al. 2014, Konstantinova et al. 2020b.

*** Marsupella boeckii (Austin) Lindb. ex Kaal. SvalbarD: Vilnet et al. 2010, Hodgetts 2015, Mamontov et al. 2018, Mamontov et al. 2019, Hodgetts \& Lockhart 2020. PRINS OSCARS LAND: Konstantinova \& Savchenko 2008a, Konstantinova \& Savchenko 2015.

*** Marsupella condensata (Ångstr. ex C.Hartm.) Lindb. ex Kaal. [Gymnomitrion condensatum Ångstr. ex C.Hartm.]. SvalBaRD: Schiffner 1893, Herzog 1926, Arnell 1928, Jørgensen 1934, Frye \& Clark 1943, Arnell 1956, Schuster \& Damsholt 1974, Schuster 1974, Schljakov 1981, Düll 1983, Smith 1990, Söderström 1995, Paton 1999, Damsholt 2002, Söderström et al. 2002a, Söderström et al. 2002b, Konstantinova 2003, Damsholt 2013, Hodgetts 2015, Hodgetts \& Lockhart 2020, Konstantinova \& Savchenko 2020b. BJøRnøYA: Berggren 1875a, Arnell \& Mårtensson 1959 [could not trace the collection reported in Berggren 1875], Söderström 1995, Frisvoll \& Elvebakk 1996, Frisvoll \& Blom 1997, Damsholt 2002, Söderström et al. 2002b. PRINS OSCARS LAND: This publication. AlBert I LAND: Arnell \& Mårtensson 1959, Frisvoll \& Blom 1992, 1997, Frisvoll \& Elvebakk 1996.

*** Marsupella sprucei (Limpr.) Bernet. Svalbard: Hodgetts 2015, Hodgetts \& Lockhart 2020. PRINS OSCARS LAND: Konstantinova \& Savchenko 2008a, 2015. NORDENSKIÖLD_LAND: Hadač 1946 [with a ?], Konstantinova \& Savchenko 2007, 2008a, 2008b.

Mesoptychia (Lindb.) A.Evans

*** Mesoptychia badensis (Gottsche ex Rabenh.) L.Söderstr. et Váňa [Lophozia badensis (Gottsche ex Rabenh.) Schiffn., Leiocolea badensis (Gottsche ex Rabenh.) Jørg.]. SValbarD: Schuster 1969, Worley 1972, Schljakov 1980, Düll 1983, Smith 1990, Söderström 1995, Paton 1999, Damsholt 2002, Söderström et al. 2002a, 2002b, Konstantinova 2003, Hodgetts 2015, Hodgetts \& Lockhart 2020. NoRDAUSTLANDET: Potemkin 2014 as Mesoptychia badensis var. apiculata. GuSTAV V LAND: Konstantinova \& Savchenko 2012 also as Leiocolea badensis var. apiculata, Konstantinova et al. 2014, Konstantinova \& Savchenko 2015 as Leiocolea badensis var. apiculata. DICKSON LAND: This publication. HAAKON VII LAND: Arnell \& Mårtensson 1959, Frisvoll \& Blom 1992, 1997. OSCAR II LAND: Arnell \& Mårtensson 1959, Frisvoll \& Blom 1992, Frisvoll \& Blom 1997. PRINS KARLS FORLAND: Konstantinova \& Savchenko 2020a. SØRKAPP LAND: This publication. WEDEL JARLSBERG LAND: Karczmarz \& Święs 1990a, Święs \& Karczmarz 1991a, Frisvoll \& Elvebakk 1996, Frisvoll \& Blom 1997.

* Mesoptychia collaris (Nees) L.Söderstr. et Váňa [Lophozia collaris (Nees) Dumort., Leiocolea collaris (Nees) Schljakov]. SVALBARD: Berggren 1875 b as Jungermannia alpestris, Bryhn 1906 as Lophozia Mülleri, Frye \& Clark 1945 as Leiocolea muelleri, Le Gallo 1951 as Leiocolea muelleri, Arnell 1956 as Leiocolea muelleri, Schuster 1969 as Lophozia collaris, Smith 1990 as Leiocolea alpestris, Söderström 1995[rejecting earlier reports], Frisvoll \& Elvebakk 1996 [rejecting earlier reports as probably $L$. heterocolpos var. arctica], Damsholt 2002 as Lophozia alpestris var. alpestris [with a “?”], Söderström et al. 2002a [rejecting earlier reports], Söderström et al. 2002b [rejecting earlier reports], Söderström et al. 2007, Damsholt 2013 as Lo- phozia alpestris, Hodgetts 2015, Hodgetts \& Lockhart 2020. GuSTAV V LAND: Konstantinova \& Savchenko 2012. BARENTSØYA: Konstantinova \& Savchenko 2018. SPITSBERGEN: Gottsche et al. 1846 as Jungermannia acuta [Bellsund], Berggren 1875a as Jungermannia muelleri [Bellsund]. DICKSON LAND: This publication. HAAKON VII LAND: This publication. NoRDENSKIÖLD LAND: Hagen 1952 as Leiocolea muelleri. PrINS KARLS ForLAND: Konstantinova \& Savchenko 2020a. SøRKAPP LAND: This publication. WEDEL JARLSBERG LAND: Konstantinova \& Koroleva 2003 as Leiocolea alpestris.

*** Mesoptychia gillmanii (Austin) L.Söderstr. et Váňa. var. gillmanii [Leiocolea gillmanii (Austin) A.Evans, Lophozia gillmanii (Austin) R.M.Schust.]. SVALBARD: Düll 1983, Söderström 1995, Paton 1999, Damsholt 2002, Söderström et al. 2002a, 2002b, Damsholt 2013, Kosovich-Anderson 2014, Hodgetts 2015, Konstantinova \& Savchenko 2020b. GuSTAV V LAND: Frisvoll \& Elvebakk 1996, Konstantinova \& Savchenko 2012. ANDRÉE LAND: Frisvoll 1981, Frisvoll \& Elvebakk 1996, Frisvoll \& Blom 1997. DICKSON_LAND: Frisvoll 1981, Frisvoll \& Blom 1997, Konstantinova \& Koroleva 2003 as Leiocolea gillmanii f. orbiculata, Konstantinova \& Savchenko 2008c, 2011. HAAKON VII LAND: Frisvoll 1981, Frisvoll \& Blom 1997, Konstantinova \& Savchenko 2006, 2008a. JAMES LAND: Frisvoll 1981, Frisvoll \& Elvebakk 1996, Frisvoll \& Blom 1997. OSCAR II LAND: Borovichev 2010, Koroleva \& Borovichev 2014. WEDEL JARLSBERG LAND: This publication.

*** Mesoptychia heterocolpos (Thed. ex Hartm.) L.Söderstr. et Váňa [Lophozia heterocolpos (Thed. ex Hartm.) M.Howe, Leiocolea heterocolpos (Thed. ex Hartm.) H.Buch]. SVALBaRD: Worley 1972, Smith 1990, Söderström 1995, Paton 1999, Söderström et al. 2002b, Damsholt 2013. DicKSON LAND: Frisvoll 1981 as cf. heterocolpos, Frisvoll \& Elvebakk 1996, Konstantinova \& Savchenko 2008c. HAAKON VII LAND: Konstantinova \& Savchenko 2006, Konstantinova \& Savchenko 2008a, Konstantinova \& Savchenko 2011. HEER LAND: Konstantinova et al. 2020b. NATHORST LAND: This publication. NORDENSKIÖLD LAND: Hadač 1989, Frisvoll \& Elvebakk 1996, Virtanen et al. 2013, Prestø et al. 2014. SøRKAPP LAND: Konstantinova et al. 2020b. TORELL LAND: Rejment-Grochowska 1967. WEDEL JARLSBERG_LAND: Rejment-Grochowska 1967, Karczmarz \& Święs 1989, Karczmarz \& Święs 1990a, Frisvoll \& Elvebakk 1996. ** — var. arctica (S.W.Arnell) L.Söderstr. et Váňa [Leiocolea heterocolpos var. arctica (S.W.Arnell) Mårtensson, Lophozia heterocolpos var. arctica (S.W.Arnell) R.M.Schust. et Damsh., Lophozia arctica (S.W.Arnell) Święs et Karczm. nom. inval.]. SVALBARD: Söderström 1995, Damsholt 2002, Söderström et al. 2002a, 2002b both, Damsholt 2003, Hodgetts 2015, Hodgetts \& Lockhart 2020. GuSTAV V LAND: This publication. DICKSON LAND: This publication. HAAKON VII LAND: Arnell \& Mårtensson 1959, Frisvoll \& Elvebakk 1996. WEEDEL_JARLSBERG_LAND: Święs \& Karczmarz $1991 b$.

** — var. harpanthoides (Bryhn et Kaal.) L.Söderstr. et Ván̆a [Leiocolea heterocolpos var. harpanthoides (Bryhn et Kaal.) R.M.Schust. ex S.W.Arnell]. S SvalBARD: Söderström et al. 2002a, Bakalin et al. 2015 [s.loc.spec.], Hodgetts 2015, Hodgetts \& Lockhart 2020. NoRDAUSTLANDET: Konstantinova \& Savchenko 2015. GuSTAV V LAND: Konstantinova \& Savchenko 2012. PRINS OSSCARS L L 
BAREnTSøya: Konstantinova \& Savchenko 2018. DiCKSON LAND: This publication. HAAKON VII LAND: Frisvoll 1978. NATHORST LAND: This publication. NoRDENSKIÖLD LAND: This publication. NYY-FrIESLAND: This publication. PRINS KARLS FORLAND: Konstantinova \& Savchenko 2020a. SøRKAPP LAND: This publication. WEDEL J lication.

** — var. heterocolpos [Lophozia heterocolpos var. heterocolpos, Leiocolea heterocolpos var. heterocolpos]. SvaLBARD: Schuster 1969, Düll 1983, Damsholt 2002, Söderström et al. 2002a, Hodgetts 2015, Hodgetts \& Lockhart 2020. BARENTSøYA: Konstantinova \& Savchenko 2018. OSSCAR II LAND: Arnell \& Mårtensson 1959.

*** Mesoptychia rutheana (Limpr.) L.Söderstr. et Váňa [Lophozia rutheana (Limpr.) M.Howe, Leiocolea rutheana (Limpr.) Müll.Frib.]. S SvALBARD: Düll 1983, Söderström 1995, Paton 1999, Damsholt 2002, Söderström et al. 2002a, 2002b, Söderström et al. 2002b, Damsholt 2013, Hodgetts 2015, Hodgetts \& Lockhart 2020. DiCKSON LAND: Frisvoll 1978, Frisvoll \& Blom 1992, 1997, Frisvoll \& Elvebakk 1996. NORDENSKIÖLD LAND: Frisvoll \& Blom 1992, 1997, Frisvoll \& Elvebakk 1996.

*** Mesoptychia sahlbergii (Lindb. et Arnell) A.Evans. SVALBARD: Düll 1983, Schuster 1983, Söderström 1995, Schuster \& Konstantinova 1996, Grolle \& Long 2000, Konstantinova 2000, Damsholt 2002, Söderström et al. 2002a, Damsholt 2013, Hodgetts 2015, Hodgetts \& Lockhart 2020, Konstantinova \& Savchenko 2020b. DICKSON_LAND: Frisvoll 1981, Frisvoll \& Blom 1992, 1997, Frisvoll \& Elvebakk 1996. NORDENSKIÖLD LAND: This publication. S SABINE_LAND: Frisvoll \& Blom 1992, Frisvoll \& Elvebakk 1996.

\section{Metzgeria Raddi}

*** Metzgeria furcata (L.) Corda. Svalbard: Hodgetts 2015, Hodgetts \& Lockhart 2020. OSCAR II LAND: Borovichev 2010. Mylia Gray

*** Mylia taylorii (Hook.) Gray [Jungermannia taylorii Hook.]. SvalbarD: Düll 1983 [with a '?'], Söderström 1995, Paton 1999, Konstantinova 2000, Damsholt 2002, Söderström et al. 2002a, 2002b, Konstantinova 2003, Damsholt 2013, Hodgetts 2015, Hodgetts \& Lockhart 2020. SPITSBERGEN: Lindberg 1866 [Kingsbay], Arnell \& Mårtensson 1959 [Kongsfjorden], Frisvoll \& Blom 1992 [Kongsfjorden], Frisvoll \& Elvebakk 1996 [Kongsfjorden], Frisvoll \& Blom 1997 [Kongsfjorden]. OSCAR II LAND: Berggren 1875a.

Nardia Gray

*** Nardia geoscyphus (De Not.) Lindb. SvalbarD: Söderström 1995, Paton 1999, Damsholt 2002, Söderström et al. 2002a, 2002b, Konstantinova 2003, Damsholt 2013, Hodgetts 2015, Hodgetts \& Lockhart 2020. Prins Oscars LAND: Konstantinova et al. 2014, Konstantinova \& Savchenko 2015. HeER LAND: This publication. NoRDENSKIÖLD LAND: Frisvoll \& Elvebakk 1996, Frisvoll \& Blom 1997, Konstantinova \& Savchenko 2007, 2008a, 2008b. OSCAR II LAND: Brattbakk et al. 1978, Frisvoll \& Elvebakk 1996, Frisvoll \& Blom 1997. WEDEL JARLSBERG LAND: Rejment-Grochowska 1967, Eurola 1968, Frisvoll \& Elvebakk 1996, Frisvoll \& Blom 1997.

Neoorthocaulis L.Söderstr., De Roo et Hedd.

** Neoorthocaulis hyperboreus (R.M.Schust.) L.Söderstr., De Roo et Hedd. Svalbard: Hodgetts \& Lockhart 2020, Konstantinova \& Savchenko 2020b. Albert I LAND: This publication. DICKSON LAND: This publication. NATHORST LAND:
This publication. NORDENSKIÖLD LAND: Ellis et al. 2016b as Barbilophozia floerkei, This publication. OSCAR II LAND: Gugnacka-Fiedor \& Nryśkiewicz 1982 as Orthocaulis floerkei, Boińska \& Gugnacka-Fiedor 1983 as Barbilophozia floerkei. PRINS KARLS ForLAND: This publication. SøøRKAPP LAND: This publication. WEEDEL J lication. NOTE: A critical taxon and the whole Neoorthocaulis-Orthocaulis-Schljakovianthus complex is in need of a molecular revision. We here report Neoorthocaulis hyperboreus as described by Damsholt (2013). Specimens reported as Barbilophozia floerkei or Orthocaulis floerkei in Ellis et al. (2016b), Gugnacka-Fiedor \& Nryśkiewicz (1982) and Boińska \& Gugnacka-Fiedor (1983) have been seen by N.A. Konstantinova and correspond to our concept of Neoorthocaulis hyperboreus, not to Neoorthocaulis floerkei.

Odontoschisma (Dumort.) Dumort.

*** Odontoschisma elongatum (Lindb.) A.Evans. SvalbarD: Schuster \& Damsholt 1974, Vilnet et al. 2012a, Hodgetts 2015, Ellis et al. 2016a, Feldberg et al. 2016, Hodgetts \& Lockhart 2020. PRINS OSCARS LAND: This publication. №RDENSKIÖLD LAND: Hadač 1989, Konstantinova \& Savchenko 2007, 2008a, 2008b, Konstantinova et al. 2020b. NOTE: Odontoschisma elongatum was rejected from Svalbard by Frisvoll \& Elvebakk (1996) but it has later been shown to occur there.

*** Odontoschisma francisci (Hook.) L.Söderstr. et Váňa [Cladopodiella francisci (Hook.) Jørg.]. SvalbarD: Schuster 1974, Düll 1983, Smith 1990, Söderström 1995, Paton 1999, Konstantinova 2000, Damsholt 2002, Söderström et al. 2002a, 2002b, Konstantinova 2003, Damsholt 2013, Hodgetts 2015, Hodgetts \& Lockhart 2020. OSCAR II LAND: Arnell \& Mårtensson 1959, Frisvoll \& Blom 1992, 1997, Frisvoll \& Elvebakk 1996.

*** Odontoschisma macounii (Austin) Underw. SvalbarD: Evans 1903, Bryhn 1906, Arnell \& Jensen 1907, Arnell 1928, Frye \& Clark 1945, Buch \& Tuomikoski 1955, Arnell 1956, Müller 1956c, Schuster et al. 1959, Schuster 1974, Bonner 1976 as Jungermannia tessellata, Düll 1983, Smith 1990, Söderström 1995, Frisvoll \& Elvebakk 1996, Paton 1999, Damsholt 2002, Söderström et al. 2002a, 2002b, Vilnet et al. 2012a, Damsholt 2013, Bakalin \& Vilnet 2014, Hodgetts 2015, Ellis et al. 2016a, Feldberg et al. 2016, Hodgetts \& Lockhart 2020. GuSTAV V LAND: This publication. PRINS OSCARS LAND: This publication. BarentSøYa: Philippi 1973, Konstantinova \& Savchenko 2018. EDGEØYA: Philippi 1973. Berggren 1875 b as Jungermannia tessellata, Arnell \& Mårtensson 1959 [W. Spitsbergen], Gradstein \& IlkiuBorges 2015 [Kings Bay]. DICKSON LAND: Konstantinova \& Savchenko 2008c. HAAKON VII LAND: Polunin 1945, Frisvoll 1978, Konstantinova \& Savchenko 2006, 2008a. NoRDENSKIÖLD LAND: Eurola 1968, Virtanen et al. 2013. OSSCAR II LAND: TYPE of Sphagnoecetis communis var. tessellata, Berggren 1875a as Sphagnoecetis communis var. tessella$t a$, Müller 1913b, Brattbakk et al. 1978, Kobayashi et al. 1990. Prins KARLS Forland: Konstantinova \& Savchenko 2020a. Wedel JARLSBERG LAND: This publication.

Oleolophozia L.Söderstr., De Roo et Hedd.

*** Oleolophozia perssonii (H.Buch et S.W.Arnell) L.Söderstr., De Roo et Hedd. [Lophozia perssonii H.Buch et S.W.Arnell]. SVALBARD: Schuster 1969, Schljakov 1980, Düll 1983, Arts 1986, Damsholt 1993, Söderström 1995, Paton 1999, Konstantinova 2000, Damsholt 2002, Söderström et 
al. 2002a, 2002b, Damsholt 2013, Hodgetts 2015, Hodgetts \& Lockhart 2020. GuSTAV V LAND: Konstantinova \& Savchenko 2012, 2015. DICKSON LAND: Konstantinova \& Savchenko 2008c. HAAKON VII LAND: Arnell \& Mårtensson 1959, Frisvoll \& Blom 1992, 1997, Frisvoll \& Elvebakk 1996. OSCAR II LAND: Arnell \& Mårtensson 1959, Frisvoll \& Blom 1992, 1997, Frisvoll \& Elvebakk 1996. PRINS KARLS FORLAND: Konstantinova \& Savchenko 2020a. Peltolepis Lindb.

*** Peltolepis quadrata (Saut.) Müll.Frib. Svalbard: Müller 1952, Arnell 1956, Steere \& Inoue 1978, Düll 1983, Söderström 1995, Damsholt 2002, Söderström et al. 2002a, 2002b, Damsholt 2013, Hodgetts 2015, Hodgetts \& Lockhart 2020. DiCKSON LAND: Frisvoll \& Elvebakk 1996, Frisvoll \& Blom 1997. HAAKON VII LAND: Frisvoll \& Elvebakk 1996, Frisvoll \& Blom 1997. JAMES LAND: Frisvoll \& Elvebakk 1996, Frisvoll \& Blom 1997. NATHORST LAND: This publication. OSCAR II LAND: Borovichev 2010, Koroleva \& Borovichev 2014. PRINS KARLS FoRLAND: Konstantinova \& Savchenko 2020a. S $ø$ RKAPP LAND: This publication. WEDEL J J LAND: This publication.

Prasanthus Lindb.

*** Prasanthus suecicus (Gottsche) Lindb. Svalbard: Schuster 1974, Schljakov 1981, Düll 1983, Söderström 1995, Schuster \& Konstantinova 1996, Konstantinova 2000, Damsholt 2002, Söderström et al. 2002a, Söderström et al. 2002b, Vilnet et al. 2010, Hodgetts 2015, Shaw et al. 2015, Patzak et al. 2016a, Patzak et al. 2016b, Mamontov et al. 2018 [s.loc.spec.], Mamontov et al. 2019, Hodgetts \& Lockhart 2020. GuSTAV V LAND: Konstantinova et al. 2020a. PRINS OSCARS LAND: Konstantinova \& Savchenko 2008a. ALBERT I LAND: Arnell \& Mårtensson 1959, Frisvoll \& Elvebakk 1996, Thingsgaard \& Damsholt 2007. HAAKON VII LAND: Arnell \& Mårtensson 1959, Frisvoll \& Elvebakk 1996. NORDENSKIÖLD LAND: Frisvoll \& Elvebakk 1996, Thingsgaard \& Damsholt 2007, Konstantinova \& Savchenko 2008b, Prestø et al. 2014. OsCAR II LAND: Arnell \& Mårtensson 1959, Frisvoll \& Elvebakk 1996. PRINS KARLS ForLAND: Konstantinova \& Savchenko 2020a. WEDEL JARLSBERG LAND: Rejment-Grochowska 1967, Karczmarz \& Święs 1990a, Frisvoll \& Elvebakk 1996.

Protochilopsis A.V.Troitsky, Bakalin et Fedosov

*** Protochilopsis grandiretis (Lindb. ex Kaal.) A.V.Troitsky, Bakalin et Fedosov [Lophozia grandiretis (Lindb. ex Kaal.) Schiffn., Schistochilopsis grandiretis (Lindb. ex Kaal.) Konstant.]. Svalbard: Schuster 1969, Schuster \& Damsholt 1974, Steere \& Inoue 1978, Schljakov 1980, Düll 1983, Bisang 1991, 1999, Söderström 1995, Damsholt 2002, Söderström et al. 2002a, 2002b, Damsholt 2013 also as Lophozia grandiretis subsp. proteidea, Hodgetts 2015, Stotler \& Crandall-Stotler 2017, Bakalin et al. 2020b, Hodgetts \& Lockhart 2020. DICKSON LAND: Konstantinova \& Savchenko 2008c as Schistochilopsis grandiretis var. parviretis, Belkina et al. 2015. HAAKON VII LAND: Konstantinova \& Savchenko 2006, 2008a. NORDENSKIÖLD LAND: Arnell \& Mårtensson 1959, Frisvoll \& Blom 1992, 1997, Frisvoll \& Elvebakk 1996. OSCAR II LAND: Koroleva \& Borovichev 2014.

Ptilidium Nees

*** Ptilidium ciliare (L.) Hampe [Jungermannia ciliaris L., Blepharozia ciliaris (L.) Dumort.]. SVALBARD: Gottsche et al. 1845, Bryhn 1906, Müller 1954a, Düll 1983, Smith
1990, Söderström 1995, Frisvoll \& Elvebakk 1996, Paton 1999, Damsholt 2002, Söderström et al. 2002a, 2002b, Hodgetts 2015, Hodgetts \& Lockhart 2020, Bakalin et al. 2021. BJøRNØYA: Berggren 1875a, Watson 1922, Summerhayes \& Elton 1923a, Söderström 1995, Frisvoll \& Elvebakk 1996, Damsholt 2002, Söderström et al. 2002b. GusTAV V LAND: Berggren 1875a, 1875b. PRINS OSCARS LAND: This publication. BARENTSøYA: Philippi 1973, Konstantinova \& Savchenko 2018. EDGEØYA: Sommerfeldt 1833, Philippi 1973, Heinemeijer 1979, Heinemeijer \& van Dijk 2004. Spitsbergen: Polunin 1945 [Kongsfjorden], Arnell \& Mårtensson 1959 [W. Spitsbergen]. AlLBERT I LAND: Lindberg 1866, Berggren 1875a, Wulff 1902 . ANDRÉE LAND: This publication. DICKSON LAND: This publication, Belkina et al. 2015. HAAKON VII LAND: Lindberg 1866, Wegener et al. 1992. JAMES LAND: This publication. NORDENSKIÖLD LAND: Watson 1922, Watson 1922 as Ptilidium ciliare f. inundatum, Hadač 1946, Hagen 1952, Eurola 1968, Hadač 1989, Konstantinova \& Savchenko 2007, 2008a, 2008b, Kreier et al. 2010, Virtanen et al. 2013, Prestø et al. 2014. OSCAR II LAND: Gugnacka-Fiedor \& Nryśkiewicz 1982, Boińska \& Gugnacka-Fiedor 1983, Kobayashi et al. 1990, Newsham \& Goodall-Copestake 2021. PRINS KARLS FORLAND: Arnell 1900, Watson 1922, Summerhayes \& Elton 1923a, 1923b, Konstantinova \& Savchenko 2020a. SøRKAPP LAND: Sommerfeldt 1833, Dubiel \& Olech 1990, Saniewski et al. 2020. WEDEL JARLSBERG LAND: Rejment-Grochowska 1967, Eurola 1968, Karczmarz \& Święs 1988, 1989, 1990a, 1990b, Święs \& Karczmarz 1991a, 1993a, 1993b, Dubiel \& Olech 1992, Kuc 1998.

Riccardia Gray

*** Riccardia chamedryfolia (With.) Grolle. NoRDENSKIÖLD LAND: This publication.

Saccobasis H.Buch

*** Saccobasis polita (Nees) H.Buch [Jungermannia polita Nees, Lophozia polita (Nees) Macoun, Sphenolobus politus (Nees) Steph., Tritomaria polita (Nees) Jørg.]. SVALBARD: Bryhn 1906, Müller 1910a, Arnell 1928, Müller 1954b, Arnell 1956, Schuster 1969, Worley 1972, Schuster \& Damsholt 1974, Schljakov 1980, Düll 1983 [with a '?"], Smith 1990, Söderström 1995, Paton 1999, Damsholt 2002 [with a “?’”, Söderström et al. 2002a, Söderström et al. 2002b, Damsholt 2013 [with a ?], Hodgetts 2015, Hodgetts \& Lockhart 2020. GuSTAV V LAND: Berggren 1875a, Frisvoll \& Elvebakk 1996. HAAKON VII LAND: This publication. NoRDENSKIÖLD LAND: This publication. OSCAR II LAND: Arnell \& Mårtensson 1959. WEDEL JARLSBERG LAND: Rejment-Grochowska 1967, Karczmarz \& Święs 1989, 1991a, Święs \& Karczmarz 1991b, 1993b, Frisvoll \& Elvebakk 1996.

** Saccobasis polymorpha (R.M.Schust.) Schljakov [Tritomaria polita subsp. polymorpha R.M.Schust.]. SVALBARD: Düll 1983 [with a '?”], Söderström 1995, Frisvoll \& Elvebakk 1996 ['probably"], Konstantinova 2000, Damsholt 2002 [with a "?"], Söderström et al. 2002a [with a '?'], Konstantinova 2003, Söderström et al. 2007, Hodgetts 2015, Hodgetts \& Lockhart 2020. HAAKON VII LAND: Konstantinova \& Savchenko 2006. PRINS KARLS FORLAND: Konstantinova \& Savchenko 2020a. S $\emptyset$ RKAPP LAND: This publication. WEDEL JARLSBERG LAND: Konstantinova \& Koroleva 2003.

\section{Sauteria Nees}

*** Sauteria alpina (Nees) Nees. SVALBARD: Lindberg 1877, 1882, Schiffner 1893, Arnell \& Jensen 1907, Müller 1907a, 
Arnell 1928, Jørgensen 1934, Arnell 1956, Worley 1972, Düll 1983, Schuster 1992, Söderström 1995, Frisvoll \& Elvebakk 1996, Damsholt 2002, Söderström et al. 2002a, 2002b, Damsholt 2013, Hodgetts 2015, Hodgetts \& Lockhart 2020. BృøRNøYA: Lindberg 1882, Kaalaas 1893a. Schiffner 1893, Schuster 1992, Söderström 1995, Frisvoll \& Elvebakk 1996, Damsholt 2002, 2013, Söderström et al. 2002b. GuSTAV V LAND: Konstantinova \& Savchenko 2012, Konstantinova et al. 2014. BARENTSøYA: Philippi 1973, Konstantinova \& Savchenko 2018. SPITSBERGEN: Arnell \& Mårtensson 1959 [W. Spitsbergen], Thingsgaard \& Damsholt 2007. Albert I LAND: Wulff 1902. BüNSOW LAND: This publication. DICKSON LAND: Konstantinova \& Savchenko 2008c, Belkina et al. 2015. HAAKON VII LAND: Konstantinova \& Savchenko 2006, 2008a, Konstantinova et al. 2020b. JAMES LAND: This publication. NORDENSKIÖLD LAND: This publication. OSCAR II LAND: Borovichev 2010, Koroleva \& Borovichev 2014. PRINS KARLS FORLAND: Konstantinova \& Savchenko 2020a. WedEL JARLSBERG LAND: Rubasinghe 2011.

Scapania (Dumort.) Dumort.

*** Scapania curta (Mårt.) Dumort. [Martinellius curtus (Mårt.) Lindb. 'Martinellia curta']. S Svalbard: Kaalaas 1893b, Müller 1901, Stephani 1910, Müller 1915a, Arnell 1928, Harmsen \& Seidenfaden 1932, Frye \& Clark 1946, Worley 1972, Schuster 1974, Schuster \& Damsholt 1974, Düll 1983, Smith 1990, Söderström 1995, Paton 1999, Damsholt 2002, Söderström et al. 2002a, 2002b, Hodgetts 2015, Hodgetts \& Lockhart 2020. BJøRnøYA: Watson 1922, Summerhayes \& Elton 1923a, Söderström 1995, Frisvoll \& Elvebakk 1996, Frisvoll \& Blom 1997, Damsholt 2002 Söderström et al. 2002b. Kong KarLS LAND: Arnell 1900, Frisvoll \& Elvebakk 1996, Frisvoll \& Blom 1997. SPITSBERGEN: Berggren 1875b. HAAKON VII LAND: Frisvoll \& Elvebakk 1996, Frisvoll \& Blom 1997. NORDENSKIÖLD LAND: Berggren 1875a [rejected as S. cuspiduligera by Arnell \& Mårtensson 1959], Müller 1905, Arnell 1922. OSCAR II LAND: Watson 1922, Summerhayes \& Elton 1923a, 1923b, Arnell \& Mårtensson 1959, Frisvoll \& Elvebakk 1996, Frisvoll \& Blom 1997. WEDEL JARLSBERG LAND: Rejment-Grochowska 1967, Święs \& Karczmarz 1991a, Frisvoll \& Elvebakk 1996, Frisvoll \& Blom 1997.

*** Scapania cuspiduligera (Nees) Müll.Frib. SVALBARD: Berggren 1875 b as Scapania bartlingii, Kaalaas 1893b as Scapania carestiae, Müller 1901 as Scapania bartlingii, Müller 1905 as Scapania bartlingii, Bryhn 1906 as Scapania bartlingii, Müller 1915b, Frye \& Clark 1946, Persson 1952, Schuster 1974, Schuster \& Damsholt 1974, Smith 1990, Söderström 1995, Paton 1999, Damsholt 2002, 2013, Söderström et al. 2002a. Hodgetts 2015, Hodgetts \& Lockhart 2020. BJøRnøYA: Berggren 1875a, 1875b both as Scapania bartlingii, Bryhn 1906 as Scapania bartlingii, Frye \& Clark 1946, Persson 1952, Söderström 1995, Frisvoll \& Elvebakk 1996, Damsholt 2002, Söderström et al. 2002b. NORDAUSTLANDET: Konstantinova \& Savchenko 2015. Prins OSSCARS LAND: This publication. BARENTSøYA: Konstantinova \& Savchenko 2018. ALBERT I LAND: TYPE of Scapania bartlingii var. elongata, TYPE of Scapania bartlingii var. obtusata, Lindberg 1866 as Scapania bartlingii var. elongate and var. obtusata, Berggren 1875a as Scapania bartlingii var. elongata, Frisvoll \& Elvebakk 1996. DickSON LAND: Frisvoll \& Elvebakk 1996, Konstantinova \& Savchenko 2008c, 2011, Belkina et al. 2015. HAAKON_VII LAND: Frisvoll \& Elve- bakk 1996, Konstantinova \& Savchenko 2006, 2008a. NATHORST LAND: This publication. NORDENSKIÖLD LAND: Berggren 1875a as Scapania bartlingii, Persson 1942, Frisvoll \& Elvebakk 1996, Konstantinova \& Savchenko 2008b. OSCAR II LAND: Koroleva \& Borovichev 2014. PRINS KARLS FORLAND: Konstantinova \& Savchenko 2020a. WEEDEL J BERG LAND: This publication.

*** Scapania gymnostomophila Kaal. Svalbard: Schuster \& Damsholt 1974, Schuster 1974, Schljakov 1981, Düll 1983, Smith 1990, Söderström 1995, Paton 1999, Damsholt 2002, 2013, Söderström et al. 2002a, 2002b, Hodgetts 2015, Hodgetts \& Lockhart 2020. BJøRNøYA: Persson 1946, Arnell 1956, Schuster 1974, Söderström et al. 2002b. NoRDAuSTLANDET: GUSTAV V LAND: Konstantinova \& Savchenko 2011, Konstantinova \& Savchenko 2012, Konstantinova \& Savchenko 2015. BARENTSØYA: Konstantinova \& Savchenko 2018. DiCKSON LAND: Frisvoll \& Blom 1992, Frisvoll \& Elvebakk 1996, Frisvoll \& Blom 1997, Konstantinova \& Koroleva 2003 as Scapania gymnostomophila f. incurva, Konstantinova \& Savchenko 2008c, Konstantinova \& Savchenko 2011. HAAKON VII LAND: Arnell \& Mårtensson 1959, Frisvoll \& Blom 1997. JAMES LAND: Frisvoll \& Blom 1992, Frisvoll \& Elvebakk 1996, Frisvoll \& Blom 1997. NATHORST LAND: This publication. OSCAR II LAND: Frisvoll \& Blom 1992. PRINS KARLS Forland: Konstantinova \& Savchenko 2020a. WEDEL JARLSBERG LAND: Konstantinova \& Koroleva 2003 as Scapania gymnostomophila f. incurva. NOTE: We are not aware of any primary report from Bjørnøya. *** Scapania hyperborea Jørg. [Martinellius hyperboreus (Jørg.) H.A.Möller 'Martinellia hyperborea']. S SvalBARD: Arnell 1928, Frye \& Clark 1946, Arnell 1956, Düll 1983, Söderström 1995, Damsholt 2002, Söderström et al. 2002a, 2002b, Damsholt 2013, Hodgetts 2015, Hodgetts \& Lockhart 2020. BuøRnøYA: Arnell 1922, 1928, Buch 1928, Harmsen \& Seidenfaden 1932, Frye \& Clark 1946, Arnell 1956, Schuster 1974 as Scapania hyperborea f. hyperborea, Söderström 1995, Frisvoll \& Elvebakk 1996, Damsholt 2002, Söderström et al. 2002b. PRINS OSCARS LAND: Konstantinova \& Savchenko 2008a. Spitsbergen: Persson 1942 [Kings Bay]. AlbBert I LAND: Arnell \& Mårtensson 1959, Frisvoll \& Elvebakk 1996. HAAKON VII LAND: Arnell \& Mårtensson 1959. NNORDENSKIÖLD LAND: Arnell 1922, Eurola 1971, Hadač 1989, Frisvoll \& Elvebakk 1996, Konstantinova \& Savchenko 2008b. OSCAR II LAND: Arnell \& Mårtensson 1959. WEDEL JARLSBERG LAND: Rejment-Grochowska 1967, Frisvoll \& Elvebakk 1996.

*** Scapania irrigua (Nees) Nees [Martinellius irriguus (Nees) Lindb. 'Martinellia irrigua']. S- Valbard: Arnell 1928, Jørgensen 1934, Frye \& Clark 1946, Arnell 1956, Müller 1956b, Worley 1972, Schuster \& Damsholt 1974, Söderström 1995, Damsholt 2002, Söderström et al. 2002b. BృøRNøYA: Watson 1922, Summerhayes \& Elton 1923a, Söderström 1995, Frisvoll \& Elvebakk 1996, Damsholt 2002, Söderström et al. 2002b. BARENTSøya: Philippi 1973, Konstantinova \& Savchenko 2018. EDGEØYA: Philippi 1973. AlBERT I LAND: Lindberg 1866 as Scapania irrigua $\gamma$ globulifera, Arnell 1922 as 'Martinellia irrigua', Frisvoll \& Elvebakk 1996. HEER LAND: Philippi 1973. NORDENSKIÖLD LAND: Hadač 1989, Frisvoll \& Elvebakk 1996. OSCAR II LAND: Brattbakk et al. 1978. SABINE LAND: Philippi 1973. WEEDEL_JARLSBERG_LAND: Rejment-Grochowska 1967, Eurola 1968, Karczmarz \& Święs 1989, Frisvoll \& Elvebakk 1996. NOTE: It is possible 
that Scapania irrigua only occur on Svalbard as subsp. rufescens.

** —subsp. irrigua. SVALBARD: Schuster 1974, Düll 1983 [with a '?'], Paton 1999, Söderström et al. 2002a [with a '?'], Hodgetts 2015, Hodgetts \& Lockhart 2020.

** - subsp. rufescens (Loeske) R.M.Schust. S $\underline{\text { ValbarD: }}$ Düll 1983 [with a '?'], Söderström 1995, Frisvoll \& Elvebakk 1996 ['.. may be the most frequent or only Svalbard representative of the aggregate species.'], Damsholt 2002 ["probably correctly recorded"], Söderström et al. 2002a, 2002b, Damsholt 2013, Hodgetts 2015, Hodgetts \& Lockhart 2020. HAAKON VII LAND: Thingsgaard \& Damsholt 2007. NORDENSKIÖLD LAND: Konstantinova \& Savchenko 2008b.

*** Scapania kaurinii Ryan. SvalbarD: Herzog 1926, Schljakov 1981, Düll 1983, Söderström 1995, Konstantinova 2000, Damsholt 2002, Söderström et al. 2002a, 2002b, Hodgetts 2015, Hodgetts \& Lockhart 2020, Konstantinova \& Savchenko 2020b. Prins OSCARS LAND: This publication. ALBERT I LAND: Arnell \& Mårtensson 1959, Frisvoll \& Elvebakk 1996. HAAKON VII LAND: Arnell \& Mårtensson 1959. OSCAR II LAND: Arnell \& Mårtensson 1959. WEDEL JaRLSBERG LAND: Rejment-Grochowska 1967, Frisvoll \& Elvebakk 1996.

*** Scapania ligulifolia R.M.Schust. [Scapania calcicola subsp. ligulifolia (R.M.Schust.) Damsh. et D.G.Long]. S- $\mathrm{vAL}=$ BARD: Düll 1983, Konstantinova 2000, Damsholt 2002, Vilnet et al. 2012a, Hodgetts 2015, Hodgetts \& Lockhart 2020, Konstantinova \& Savchenko 2020b. GustaV V LAND: Konstantinova \& Savchenko 2011, 2012, 2015. PRINS_OSCARS LAND: This publication. DICKSON LAND: Konstantinova \& Savchenko 2011. HAAKON VII LAND: Konstantinova \& Savchenko 2011. NATHORST LAND: This publication. PRINS KARLS_FORLAND: Konstantinova \& Savchenko 2020a. SøRKAPP LAND: This publication. WedeL JARLSBERG LAND: This publication.

*** Scapania obcordata (Berggr.) S.W.Arnell [Sarcocyphos obcordatus Berggr. 'Sarcoscyphus'. Marsupella obcordata (Berggr.) Steph.]. Svalbard: Stephani 1901a, Persson 1962, Lammes 1977, Steere \& Inoue 1978, Schljakov 1981, Düll 1983, Geissler \& Bischler 1985, Ochyra \& Váňa 1989, Geissler \& Bischler 1990, Söderström 1995, Bednarek-Ochyra et al. 2000, Söderström et al. 2002a, 2002b, Hässel \& Rubies 2009, Vilnet et al. 2010, 2012a, Ellis et al. 2014b, Hodgetts 2015, Borovichev et al. 2016, Hodgetts \& Lockhart 2020. BJøRNøYA: SYNTYPE, Söderström 1995, Frisvoll \& Elvebakk 1996, Damsholt 2002, Söderström et al. 2002b. GuSTAV V LAND: SYNTYPE, Berggren 1875a, Lindberg \& Arnell 1889, Schuster 1974, Grolle 1976, Frisvoll \& Elvebakk 1996, Damsholt 2002, 2013, Konstantinova \& Savchenko 2012. PRINS OSCARS LAND: Konstantinova \& Savchenko 2008a, 2020b. BARENTSØYA: Konstantinova $\&$ Savchenko 2018. Albert I LAND: Arnell \& Mårtensson 1959. DICKSON LAND: This publication. HAAKON_VII LAND: This publication. HEER LAND: This publication. JAMES LAND: This publication. №RDENSKIÖLD LAND: Arnell \& Mårtensson 1959, Hadač 1989, Frisvoll \& Elvebakk 1996, Potemkin 1999, Konstantinova \& Savchenko 2007, 2008a, 2008b, Heinrichs et al. 2012, Konstantinova et al. 2020b. OSCAR II LAND: Arnell \& Mårtensson 1959. PRINS KARLS FORLAND: Konstantinova \& Savchenko 2020a. SøRKAPP LAND: Stebel et al. 2018. WEDEL J
1967, Karczmarz \& Święs 1989, 1990a, Frisvoll \& Elvebakk 1996.

*** Scapania paludicola Loeske et Müll.Frib. var. paludicola [Martinellius paludicola (Loeske et Müll.Frib.) C.E.O.Jensen 'Martinellia']. SvalbarD: Arnell 1928, Buch 1928, Jørgensen 1934, Frye \& Clark 1946, Arnell 1956, Worley 1972, Schuster 1974, Düll 1983, Smith 1990, Söderström 1995, Paton 1999, Damsholt 2002, 2013, Söderström et al. 2002a, 2002b, Hodgetts 2015, Hodgetts \& Lockhart 2020. PRINS OSCARS LAND: This publication. ALBERT I LAND: Arnell 1922, Frisvoll \& Elvebakk 1996. NATHORST LAND: This publication. NORDENSKIÖLD LAND: Eurola 1971, Hadač 1989, Frisvoll \& Elvebakk 1996. OSCAR II LAND: Kobayashi et al. 1990, Frisvoll \& Elvebakk 1996. WEDEL_JARLSBERG_LAND: Rejment-Grochowska 1967, Frisvoll \& Elvebakk 1996.

** Scapania parvifolia Warnst. [Scapania scandica $\mathrm{f}$. parvifolia (Warnst.) Schljakov]. SvalBARD: Schuster 1974, Düll 1983, Smith 1990, Söderström 1995, Paton 1999, Damsholt 2002, Damsholt 2013, Ellis et al. 2014b, Hodgetts 2015, Hodgetts \& Lockhart 2020. SPITSBERGEN: Schuster \& Damsholt 1974. ALBERT I LAND: Arnell \& Mårtensson 1959, Frisvoll \& Blom 1992, 1997, Frisvoll \& Elvebakk 1996 [with some doubt], Thingsgaard \& Damsholt 2007. NORDENSKIÖLD LAND: Konstantinova \& Savchenko 2007, 2008b.

*** Scapania praetervisa Meyl. [Scapania mucronata subsp. praetervisa (Meyl.) Schljakov, Scapania mucronata var. praetervisa (Warnst.) Konstant. et Czernjad. nom. inval.]. SVALBARD: Persson 1962, Lammes 1977, Düll 1983, Smith 1990, Söderström 1995, Frisvoll \& Blom 1997 as Scapania mucronata [given as $S$. mucronata s. lat. but restricted in a note], Paton 1999, Damsholt 2002, Söderström et al. 2002a, 2002b, Hodgetts 2015, Hodgetts \& Lockhart 2020. DiCKSON LAND: This publication. HAAKON VII LAND: Arnell \& Mårtensson 1959. J JAMES LAND: This publication. NORDENSKIÖLD LAND: This publication. OSCAR II LAND: Arnell \& Mårtensson 1959.

*** Scapania simmonsii Bryhn et Kaal. SvalBard: Buch 1933, Arnell 1950, Steere 1953, Arnell 1956, Schuster 1974, Godfrey \& Godfrey 1978, Godfrey \& Schofield 1979, Schljakov 1981, Düll 1983, Frisvoll \& Blom 1992, 1997, Söderström 1995, Frisvoll \& Elvebakk 1996, Konstantinova 2000, Damsholt 2002, 2013, Söderström et al. 2002a, 2002b, Hodgetts 2015, Hodgetts \& Lockhart 2020, Konstantinova \& Savchenko 2020b. NOTE: The only report is old and without locality and collector specified (only "det. Kaalaas, affirm. H. Buch").

*** Scapania spitsbergensis (Lindb.) Müll.Frib. [Martinellius spitsbergensis Lindb. 'Martinellia']. SVvaLbarD: Müller 1900, Müller 1901, Arnell \& Jensen 1907, Stephani 1910, Evans 1911, Arnell 1928, Frye \& Clark 1946, Steere 1954, Arnell 1956, Müller 1956b, Schuster \& Damsholt 1974, Steere \& Inoue 1978, Schljakov 1981, Düll 1983, Schuster 1983, Wegener et al. 1992, Söderström 1995, Konstantinova 2000, Söderström et al. 2002a, 2002b, Vilnet et al. 2010, Heinrichs et al. 2012, Hodgetts 2015, Zhang et al. 2017, Hodgetts \& Lockhart 2020. GuSTAV V LAND: Konstantinova et al. 2020b. PRINS OSCARS LAND: Konstantinova \& Savchenko 2008a, Klimova \& Bakalin 2017. ALBERT I LAND: TYPE, Berggren 1875a as Scapania nemorea, Lindberg \& Arnell 1889, Müller 1905, Müller 1915b, Arnell 1922, Buch 1928, Arnell \& Mårtensson 1959, Schuster 1974, Grolle 1976, Geissler \& Bischler 1985, Frisvoll \& Blom 1992, Potemkin 
1994, Frisvoll \& Elvebakk 1996, Frisvoll \& Blom 1997, Damsholt 2002, 2013, Konstantinova \& Savchenko 2008a. HAAKON VII LAND: Arnell \& Mårtensson 1959, Frisvoll \& Blom 1992. NORDENSKIÖLD LAND: Konstantinova \& Savchenko 2008a. OSCAR II LAND: Frisvoll \& Elvebakk 1996, Frisvoll \& Blom 1997. WEDEL JARLSBERG LAND: Rejment-Grochowska 1967, Frisvoll \& Elvebakk 1996, Frisvoll \& Blom 1997.

*** Scapania subalpina (Nees ex Lindenb.) Dumort. SvaLBARD: Kaalaas 1893b, Düll 1983, Smith 1990, Söderström 1995, Paton 1999, Damsholt 2002, Söderström et al. 2002a, 2002b, Damsholt 2013, Hodgetts 2015, Hodgetts \& Lockhart 2020. WEDEL JARLSBERG LAND: Rejment-Grochowska 1967, Karczmarz \& Święs 1989, Frisvoll \& Blom 1992, 1997, Frisvoll \& Elvebakk 1996.

*** Scapania tundrae (Arnell) H.Buch [Scapania hyperborea var. tundrae (Arnell) Potemkin]. SvalbarD: Jørgensen 1934, Arnell 1956, Müller 1956b, Schuster \& Damsholt 1974, Schljakov 1981, Düll 1983, Söderström 1995, Konstantinova 2000, Damsholt 2002, Söderström et al. 2002a, 2002b, Vilnet et al. 2010, Heinrichs et al. 2012, Hodgetts 2015, Hodgetts \& Lockhart 2020. NORDAUSTLANDET: Konstantinova \& Savchenko 2015. Prins OSCARS LAND: This publication. Albert I Land: TYPE of Scapania berggrenii, Arnell \& Mårtensson 1959, Schuster 1974, Frisvoll \& Elvebakk 1996, Potemkin 1999, Thingsgaard \& Damsholt 2007. HAAKON VII LAND: This publication. NORDENSKIÖLD LAND: Buch 1928, Frisvoll \& Elvebakk 1996, Konstantinova \& Savchenko 2007, 2008a, 2008b, Damsholt 2013, Konstantinova et al. 2020b. OSCAR II LAND: Arnell \& Mårtensson 1959. PRINS KARLS FoRLAND: Konstantinova \& Savchenko 2020a. SøRKAPP LAND: Dubiel \& Olech 1990, Frisvoll \& Elvebakk 1996. WEDEL JARLSBERG LAND: Rejment-Grochowska 1967, Eurola 1968, Karczmarz \& Święs 1989, Frisvoll \& Elvebakk 1996.

*** Scapania uliginosa (Lindenb.) Dumort. SVAlbard: Söderström et al. 2002a, Konstantinova 2003, Damsholt 2013, Ellis et al. 2015b, Hodgetts 2015, Stotler \& CrandallStotler 2017, Hodgetts \& Lockhart 2020. NY-FrIESLAND: Karlsson 1993, Frisvoll \& Elvebakk 1996, Frisvoll \& Blom 1997.

Schistochilopsis (N.Kitag.) Konstant.

* Schistochilopsis hyperarctica Konstant. et L.Söderstr. [Lophozia hyperarctica R.M.Schust. nom. inval.]. SVALBARD: Düll 1983, Söderström 1995, Grolle \& Long 2000, Konstantinova 2000, Damsholt 2002, Söderström et al. 2002a, 2002b, Ellis et al. 2013a, Hodgetts 2015, Hodgetts \& Lockhart 2020. SPITSBERGEN: Frisvoll \& Elvebakk 1996 [Dicksonfjorden]. JAMES LAND: Frisvoll 1981, Bisang 1991 [with some doubt], Frisvoll \& Blom 1992, 1997.

*** Schistochilopsis incisa (Schrad.) Konstant. [Lophozia incisa (Schrad.) Dumort.]. SvalbaRD: Schuster 1969, Worley 1972, Düll 1983, Gradstein \& Váňa 1987, Smith 1990, Söderström 1995, Damsholt 2002. Barentsøya: Philippi 1973, Konstantinova \& Savchenko 2018. AlBERT I LAND: Arnell \& Mårtensson 1959. HEER LAND: Philippi 1973.

* Schistochilopsis opacifolia (Culm. ex Meyl.) Konstant. [Lophozia opacifolia (Culm. ex Meyl.) Meyl., Lophozia incisa subsp. opacifolia (Culm. ex Meyl.) R.M.Schust. et Damsh. nom. inval.]. SVALBARD: Düll 1983, Bisang 1991, Söderström 1995, Paton 1999, Damsholt 2002, Söderström et al. 2002a, 2002b, Vilnet et al. 2010, Damsholt 2013, Hodgetts
2015, Hodgetts \& Lockhart 2020. ?BARENTSøYA: Frisvoll \& Elvebakk 1996 . ?ALBERT I LAND: Frisvoll \& Elvebakk 1996. DiCKSON LAND: Frisvoll 1981. HAAKON VII LAND: Frisvoll 1978, 1981, Frisvoll \& Elvebakk 1996. ?HEER LAND: Frisvoll \& Elvebakk 1996 . JAMES LAND: Frisvoll 1981, Frisvoll \& Elvebakk 1996. NORDENSKIÖLD LAND: Konstantinova \& Savchenko 2007, 2008a, 2008b, Konstantinova et al. 2020b. NOTE: We are not aware of any primary report from Barentsøya, Albert I Land and Heer Land. Frisvoll \& Elvebakk (1996) only assumed that the reports of Lophozia incisa belongs to Schistochilopsis opacifolia.

Schizophyllopsis Váňa et L.Söderstr.

*** Schizophyllopsis sphenoloboides (R.M.Schust.) Váňa et L.Söderstr. [Anastrophyllum sphenoloboides R.M.Schust., Schizophyllum sphenoloboides (R.M.Schust.) Váňa et L.Söderstr.]. SVALBARD: Vilnet et al. 2010, 2012a, Damsholt 2013, Mamontov \& Vilnet 2013, Hodgetts 2015, Hodgetts \& Lockhart 2020. NORDENSKIÖLD LAND: Konstantinova \& Savchenko 2008a, 2008b, Konstantinova \& Savchenko 2020 b.

\section{Schljakovia Konstant. et Vilnet}

*** Schljakovia kunzeana (Huebener) Konstant. et Vilnet. [Lophozia kunzeana (Huebener) A. Evans, Orthocaulis kunzeanus (Huebener) H.Buch, Barbilophozia kunzeana (Huebener) Müll.Frib.]. SVALBARD: Berggren $1875 \mathrm{~b}$ as Jungermannia plicata $\gamma$ gracilis, Kaalaas 1893 c as Jungermannia kunzei var. plicata, Bryhn 1906, Frye \& Clark 1945, Müller 1954a, Arnell 1956, Düll 1983, Smith 1990, Söderström 1995, Frisvoll \& Elvebakk 1996, Paton 1999, Damsholt 2002, 2013, Söderström et al. 2002a, Hodgetts 2015, Hodgetts \& Lockhart 2020. ALBERT I LAND: TYPE of Jungermannia plicata var. gracilis, Berggren 1875a as Jungermannia plicata, Berggren 1875a as Jungermannia plicata var. gracilis, Geissler \& Bischler 1987 as Jungermannia plicata var. gracilis. DiCKSON LAND: This publication. NATHORST LAND: This publication. NORDENSKIÖLD LAND: Hagen 1952, Konstantinova \& Savchenko 2008b, Virtanen et al. 2013, Konstantinova et al. 2020b. OSCAR II LAND: Watson 1922, Summerhayes \& Elton 1923a, 1923b. SøRKAPP LAND: This publication. Wedel_JARLSBERG LAND: This publication.

Schljakovianthus Konstant. et Vilnet

*** Schljakovianthus quadrilobus (Lindb.) Konstant. et Vilnet [Jungermannia quadriloba Lindb., Barbilophozia quadriloba (Lindb.) Loeske, Lophozia quadriloba (Lindb.) A.Evans, Orthocaulis quadrilobus (Lindb.) H.Buch]. SvaLBARD: Evans 1900, Bryhn 1906, Müller 1910b, Arnell 1928, Jørgensen 1934, Frye \& Clark 1945, Müller 1954a, Arnell 1956, Schuster 1969, Worley 1972, Düll 1983, Smith 1990, Söderström 1995, Söderström 1995 as Lophozia quadriloba var. glareosa, Frisvoll \& Elvebakk 1996, Paton 1999, Damsholt 2002, Söderström et al. 2002a, 2002b, Hodgetts 2015, Hodgetts \& Lockhart 2020. GuSTAV V LAND: Konstantinova \& Savchenko 2012 also as Schljakovianthus quadrilobus var. glareosus, Konstantinova et al. 2014. PRINS_OSCARS LAND: Konstantinova et al. 2014. BARENTSØYA: Philippi 1973, Konstantinova \& Savchenko 2018. EDGEØYA: Philippi 1973. Kong KarLS LAND: Arnell 1900. SPITSBERGEN: Arnell \& Mårtensson 1959 also as Orthocaulis quadrilobus var. glareosa [W. Spitsbergen]. ALBERT I LAND: TYPE of Jungermannia floerkei F.Weber et D.Mohr $\delta$ gemmipara, Lindberg 1866 as Jungermannia floerkei F. Weber et D.Mohr ä gemmipara. ANDRÉE LAND: This publication. DICKSON LAND: 
Konstantinova \& Savchenko 2008c, Konstantinova \& Savchenko 2011, Belkina et al. 2015. HAAKON VII LAND: Konstantinova \& Savchenko 2006, Newsham \& GoodallCopestake 2021. HEER LAND: Konstantinova et al. 2020b. JAMES LAND: This publication. NATHORST LAND: This publication. NoRdenskiöld LAND: Persson 1942, Hagen 1952, Eurola 1971, Konstantinova \& Savchenko 2007, 2008b, Konstantinova et al. 2020b. OSCAR II LAND: Kobayashi et al. 1990 as Barbilophozia quadriloba var. glareosa, Newsham \& Goodall-Copestake 2021. PRINS KARLS_FORLAND: Müller 1910b, Konstantinova \& Savchenko 2020a as Schljakovianthus quadrilobus var. glareosus. S-ABINE LAND: Philippi 1973, Konstantinova et al. 2014. SøRKAPP LAND: This publication. WEDEL_JARLSBERG_LAND: Rejment-Grochowska 1967, Karczmarz \& Święs 1989.

Solenostoma Mitt.

*** Solenostoma confertissimum (Nees) Schljakov [Jungermannia confertissima Nees]. SVALbarD: Schljakov 1981, Smith 1990, Söderström 1995, Damsholt 2002, Söderström et al. 2002a, Damsholt 2013, Hodgetts 2015, Hodgetts \& Lockhart 2020. NORDENSKIÖLD LAND: This publication. OSCAR II LAND: Váňa 1974, Frisvoll \& Blom 1992, 1997, Frisvoll \& Elvebakk 1996, Minami et al. 1997b.

*** Solenostoma obovatum (Nees) C.Massal. [Jungermannia obovata Nees, Plectocolea obovata (Nees) Mitt.]. SvaLBARD: Buch \& Fagerström 1946, Schuster 1969, Schuster 1969 as Solenostoma subellipticum, Worley 1972, Schuster \& Damsholt 1974 as Solenostoma subellipticum, Váňa 1975, Schljakov 1981, Düll 1983 also as Jungermannia subelliptica, Smith 1990 as Jungermannia subelliptica, Söderström 1995 also as Jungermannia obovata subsp. minor, Frisvoll \& Elvebakk 1996, Paton 1999 as Jungermannia subelliptica, Damsholt 2002 also as Jungermannia obovata subsp. minor, Söderström et al. 2002a as Jungermannia subellipti$c a$, Söderström et al. 2002a [rejecting earlier reports], Söderström and Jungermannia subelliptica, Damsholt 2013 also as Jungermannia obovata subsp. minor, Hodgetts 2015 as Solenostoma subellipticum, Hodgetts \& Lockhart 2020 as Solenostoma subellipticum, Kusnetsova et al. 2020 as Plectocolea subelliptica. DICKSON_LAND: This publication. HAAKON VII LAND: Arnell \& Mårtensson 1959 also as Plectocolea subelliptica. NATHORST LAND: This publication. NoRDENSKIÖLD LAND: Arnell \& Mårtensson 1959 as Plectocolea subelliptica, Váňa 1975 as Jungermannia subelliptica, Frisvoll \& Blom 1992, Frisvoll \& Elvebakk 1996 as Jungermannia subelliptica, Konstantinova \& Savchenko 2007, 2008a, 2008b all as Plectocolea subelliptica. OSCAR II LAND: Arnell \& Mårtensson 1959 also as Plectocolea subelliptica, Váňa 1975 as Jungermannia subelliptica, Frisvoll \& Blom 1992, Frisvoll \& Elvebakk 1996 as Jungermannia subelliptica. PRINS KARLS ForLAND: Konstantinova \& Savchenko 2020a as Solenostoma subellipticum. S $\underline{\text { } R K A P P} \underline{\text { LAND}}$ : This publication. WEDEL JARLSBERG LAND: This publication. NOTE: All reports of Solenostoma obovatum from Svalbard can be referred to Solenostoma subellipticum which we here regard as a synonym.

*** Solenostoma sphaerocarpum (Hook.) Steph. [Jungermannia sphaerocarpa Hook.]. SVALBARD: Buch \& Fagerström 1946 as Jungermannia jenseniana, Váňa 1974 also as Jungermannia jenseniana, Schljakov 1981, Smith 1990, Söderström 1995 also as Jungermannia jenseniana, Frisvoll \& Elvebakk 1996 as Jungermannia jenseniana, Paton 1999, Damsholt
2002 also as Jungermannia jenseniana, Söderström et al. 2002a, 2002b, Damsholt 2013, Hodgetts 2015 as Solenostoma pusillum, Hodgetts 2015, Hodgetts \& Lockhart 2020, Kusnetsova et al. 2020. GuSTAV V LAND: Konstantinova \& Savchenko 2012 as Solenostoma cf. sphaerocarpum var. nanum. DICKSON LAND: This publication. NORDENSKIÖLD LAND: This publication. (OSCAR II LAND: Arnell \& Mårtensson 1959 as Jungermannia pusilla [rejected as Jungermannia confertissima by Frisvoll \& Elvebakk 1996]). PRINS KARLS FORLAND: Konstantinova \& Savchenko 2020a. SLAND: Dubiel \& Olech 1990 as Solenostoma 'sphaerocarpa var. nana', Frisvoll \& Blom 1992, 1997 both as Jungermannia sphaerocarpa var. nana, Frisvoll \& Elvebakk 1996. WEDEL_JARLSBERG LAND: Rejment-Grochowska 1967 as Solenostoma sphaerocarpum var. 'nana', Karczmarz \& Święs 1988, 1989 both as Solenostoma sphaerocarpum var. nanum, Święs \& Karczmarz 1991a, 1991b as Solenostoma sphaerocarpum var. 'nana', Frisvoll \& Blom 1992, 1997 both as Jungermannia sphaerocarpa var. nana, Frisvoll \& Elvebakk 1996. NOTE: We are not aware of any correctly identified report from Oscar II Land and only reported with some doubt from Gustav V Land.

Sphenolobus (Lindb.) Berggr.

*** Sphenolobus minutus (Schreb.) Berggr. [Jungermannia minuta Schreb., Anastrophyllum minutum (Schreb.) R.M.Schust.]. S VALBARD: Stephani 1902, Bryhn 1906, Müller 1909c, Herzog 1926 as Sphenolobus saccatulus, Harmsen \& Seidenfaden 1932, Jørgensen 1934 as Sphenolobus minutus var. grandis, Frye \& Clark 1945 as Sphenolobus minutus var. grandis, Frye \& Clark 1945, Müller 1954b, Arnell 1956, Schuster 1969 as Anastrophyllum minutum var. weberi, Worley 1972, Smith 1990, Söderström 1995 also as Anastrophyllum minutum var. weberi, Frisvoll \& Elvebakk 1996, Paton 1999, Damsholt 2002 also as Anastrophyllum minutum var. weberi, Söderström et al. 2002a, 2002b both also as Anastrophyllum minutum var. weberi, Schill \& Long 2003, Hentschel et al. 2006, Heinrichs et al. 2007, Hentschel et al. 2007, Feldberg et al. 2009, 2010, 2013, Vilnet et al. 2010, 2012a, Damsholt 2013 as Anastrophyllum minutum var. weberi, Mamontov \& Vilnet 2013, 2017, Stotler \& Crandall-Stotler 2017, Hodgetts \& Lockhart 2020. GuSTAV V LAND: Hooker 1828, Berggren 1875a, 1875b, Konstantinova \& Savchenko 2012. PRINS OSCARS LAND: Konstantinova \& Savchenko 2008a. BARENTSøYa: Philippi 1973, Konstantinova \& Savchenko 2018. EDGEØYA: Philippi 1973. Kvitøya: This publication. SPITSBERGEN: Gottsche et al. 1847 [Bellsund], Arnell \& Mårtensson 1959 as Sphenolobus minutus var. grandis [W. Spitsbergen]. ALBERT I LAND: Lindberg 1866 as Jungermannia minuta 1 fasciculata, Berggren 1875a, Thingsgaard \& Damsholt 2007. DICKSON LAND: This publication. HAAKON VII LAND: Wegener et al. 1992, Thingsgaard \& Damsholt 2007, Newsham \& GoodallCopestake 2021. JAMES LAND: This publication. NNORDENSKIÖLD LAND: Berggren 1875a, Hagen 1952, Eurola 1968, Hadač 1989, Konstantinova \& Savchenko 2007, 2008a, 2008b, Stebel et al. 2018 as Sphenolobus minutus var. grandis. NY-FrIESLAND: This publication. OSCAR II LAND: Kobayashi et al. 1990, Newsham \& Goodall-Copestake 2021. PRINS KARLS ForLAND: Hagen 1908, Watson 1922, Summerhayes \& Elton 1923a, 1923b, Konstantinova \& Savchenko 2020a. SABIne LAND: Philippi 1973. Torell LAND: Rejment-Grochowska 1967. WEDEL_JARLSBERG LAND: Rejment- 
Grochowska 1967, Karczmarz \& Święs 1988, 1989, 1990a, 1990b, Święs \& Karczmarz 1991a, 1991b, 1993b.

Tetralophozia (R.M.Schust.) Schljakov

*** Tetralophozia setiformis (Ehrh.) Schljakov [Jungermannia setiformis Ehrh., Chandonanthus setiformis (Ehrh.) Lindb., Temnoma setiforme (Ehrh.) M.Howe, Blepharostoma setiforme (Ehrh.) Schiffn.]. SvalBaRD: Bryhn 1906, Müller 1914a, Arnell 1928, Harmsen \& Seidenfaden 1932, Frye \& Clark 1945, Müller 1954a, Steere 1954, Arnell 1956, Bird \& Ogilvie 1964, Schuster 1969, Worley 1972, Schuster \& Damsholt 1974, Düll 1983, Söderström 1995, Frisvoll \& Elvebakk 1996, Paton 1999, Damsholt 2002, 2013, Söderström et al. 2002a, 2002b, Hodgetts 2015, Hodgetts \& Lockhart 2020, Konstantinova \& Savchenko 2020b. GuSTAV _ V LAND: Lindberg 1866 as Jungermannia setiformis var. alpinus, Berggren 1875a, Berggren 1875b. PRINS OSCARS LAND: This publication. Barentsøya: Philippi 1973, Konstantinova \& Savchenko 2018. ALBERT I LAND: Gottsche et al. 1847 as Jungermannia setiformis var. alpinus, Livesay 1870, Berggren 1875a, Arnell \& Mårtensson 1959, Thingsgaard \& Damsholt 2007. HAAKON VII LAND: Lindberg 1866 as Jungermannia setiformis var. alpinus, Berggren 1875a, Arnell \& Mårtensson 1959, Inoue 1988, Wegener et al. 1992. NORDENSKIÖLD LAND: Hadač 1946, Eurola 1968. OCAR II LAND: Arnell \& Mårtensson 1959. PRINS KARLS FoRLAND: Konstantinova \& Savchenko 2020a. S Dubiel \& Olech 1990. WEDEL JARLSBERG LAND: RejmentGrochowska 1967, Bednarek-Ochyra et al. 1987a, Karczmarz \& Święs 1988, 1991a, Kuc 1998.

\section{Trilophozia (R.M.Schust.) Bakalin}

*** Trilophozia quinquedentata (Huds.) Bakalin [Jungermannia quinquedentata Huds., Lophozia quinquedentata (Huds.) Cogn., Tritomaria quinquedentata (Huds.) H.Buch]. SvalBarD: Bryhn 1906, Frye \& Clark 1945, Arnell 1956, Schuster 1958, Schuster 1969, Worley 1972, Düll 1983, Persson \& Viereck 1983 as Tritomaria quinquedentata subsp. turgida, Geissler \& Bischler 1987 of Jungermannia attenuata $\beta^{*}$ laxifolia, Smith 1990, Söderström 1995 also as Tritomaria quinquedentata subsp. turgida, Paton 1999, Damsholt 2002 also as Tritomaria quinquedentata subsp. turgi$d a$, Söderström et al. 2002a, 2002b both also as Tritomaria quinquedentata subsp. turgida, Vilnet et al. 2010, 2012a both as Tritomaria quinquedentata f. gracilis, Damsholt 2013 as Tritomaria quinquedentata subsp. turgida, Hodgetts 2015, Bakalin \& Vilnet 2019 as Tritomaria quinquedentata f. gracilis, Hodgetts \& Lockhart 2020, Konstantinova \& Savchenko 2020b, Konstantinova et al. 2020a as Trilophozia quinquedentata f. gracilis. B 1922, Summerhayes \& Elton 1923a, Söderström 1995, Frisvoll \& Elvebakk 1996, Damsholt 2002, Söderström et al. 2002b. GUSTAV V LAND: Lindberg 1866, Berggren 1875a, Konstantinova \& Savchenko 2012. PRINS OSCARS_LAND: Konstantinova \& Savchenko 2008a, Konstantinova et al. 2014. BARENTSøya: Hoffman 1968, Philippi 1973, Konstantinova \& Savchenko 2018 also as Trilophozia quinquedentata var. turgida. EDGEØYA: Philippi 1973, Heinemeijer 1979, Heinemeijer \& van Dijk 2004. KONG KARLS LAND: Arnell 1900, Hoffman 1968. Kvitøya: Hoffman 1968. SpitsBergen: Arnell \& Mårtensson 1959. ALBERT I LAND: TYPE of Jungermannia attenuata $\beta^{*}$ laxifolia, Lindberg 1866 of Jungermannia attenuata $\beta^{*}$ laxifolia, Thingsgaard \& Damsholt 2007 as Tritomaria quinquedentata f. gracilis, Konstanti- nova \& Savchenko 2008a. DICKSON LAND: Konstantinova \& Savchenko 2008c, 2011, Belkina et al. 2015, Konstantinova et al. 2020b. HAAKON VII LAND: Lindberg 1866, Berggren 1875a, Wegener et al. 1992, Konstantinova \& Savchenko 2006, 2008a, Thingsgaard \& Damsholt 2007 as Tritomaria quinquedentata subsp. turgida, Newsham \& Goodall-Copestake 2021. HeER LAND: Philippi 1973. NoRDENSKIÖLD LAND: Watson 1922, Hadač 1946, 1989, Hagen 1952, Konstantinova \& Savchenko 2007, 2008a, 2008b, Thingsgaard \& Damsholt 2007 as Tritomaria quinquedentata f. gracilis, Virtanen et al. 2013, Stebel et al. 2018 as Trilophozia quinquedentata var. turgida, Konstantinova et al. 2020b. OSCAR II LAND: Watson 1922 as Lophozia quinquedentata var. turgida, Summerhayes \& Elton 1923a,1923b, Arnell \& Mårtensson 1959 as Tritomaria quinquedentata var. turgida, Kobayashi et al. 1990, Frisvoll \& Elvebakk 1996 as Tritomaria quinquedentata var. turgida, Newsham \& Goodall-Copestake 2021. $\underline{\text { PRINS KARLS }}$ FORLAND: Hagen 1908, Watson 1922, Summerhayes \& Elton 1923a, 1923b, Harmsen \& Seidenfaden 1932, Konstantinova \& Savchenko 2020a. WedEL JARLSBERG LAND: Rejment-Grochowska 1967, Eurola 1968, Karczmarz \& Święs 1988, 1989, Święs \& Karczmarz 1991a, 1991b, Kuc 1998. Tritomaria Schiffn. ex Loeske

*** Tritomaria exsectiformis (Breidl.) Schiffn. ex Loeske. SVAlbard: Düll 1983, Söderström 1995, Paton 1999, Söderström et al. 2002a, 2002b, Konstantinova 2003. OSCAR II LAND: Arnell \& Mårtensson 1959 ['may represent ssp. arctica' fide Schuster 1969], Frisvoll \& Blom 1992, 1997, Frisvoll \& Elvebakk 1996 ["probably subsp. exsectiformis"].

** - subsp. exsectiformis. SVAlBarD: Schuster 1969, Söderström 1995, Damsholt 2002, Hodgetts 2015, Hodgetts \& Lockhart 2020.

*** Tritomaria scitula (Taylor) Jørg. SvalbarD: Düll 1983, Söderström 1995, Frisvoll \& Elvebakk 1996, Damsholt 2002, Söderström et al. 2002a, 2002b, Damsholt 2013, Hodgetts 2015, Hodgetts \& Lockhart 2020. GusTAV V LAND: Konstantinova \& Savchenko 2012. SPITSBERGEN: Thingsgaard \& Damsholt 2007. DiCKSON LAND: Konstantinova \& Savchenko 2008c, 2011. HAAKON VII LAND: Arnell \& Mårtensson 1959, Konstantinova \& Savchenko 2006, 2008a. HEER LAND: Konstantinova et al. 2020b. JAMES LAND: This publication. NATHORST LAND: This publication. NORDENSKIÖLD LAND: Hagen 1952, Eurola 1968, Hadač 1989. OQSCAR II LAND: Arnell \& Mårtensson 1959, Brattbakk et al. 1978, Kobayashi et al. 1990, Koroleva \& Borovichev 2014. PRINS KARLS FORLAND: Konstantinova \& Savchenko 2020a. SøRKAPP LAND: This publication. WEDEL JARLSBERG_LAND: Rejment-Grochowska 1967, Karczmarz \& Święs 1988, 1989, Święs \& Karczmarz 1991a, 1991b.

TAXA ONLY DOUBTFULLY OCCURING ON SVALBARD Anthelia (Dumort.) Dumort.

*** Anthelia julacea (L.) Dumort. [Jungermannia julacea L.]. Svalbard: Phipps 1774, Phips 1777, Berggren 1875b, Spruce 1882, Harmsen \& Seidenfaden 1932, Frye \& Clark 1943, Le Gallo 1951, Steere 1954, Schuster 1974, Düll 1983, Smith 1990, Söderström 1995, Frisvoll \& Elvebakk 1996, Paton 1999, Damsholt 2002, Söderström et al. 2002a, Söderström et al. 2002b [with a '?'], Konstantinova 2003 [with doubt], Damsholt 2013, Hodgetts 2015, Hodgetts \& Lockhart 2020. BJøRNøYA: Berggren 1875a. KONG KARLS LAND: Arnell 1900. AlBERT I LAND: Berggren 1875a. PRINS KARLS 
FORLAND: Berggren 1875a. WEDEL JARLSBERG LAND: RejmentGrochowska 1967, Karczmarz \& Święs 1989, Frisvoll \& Blom 1992, Frisvoll \& Blom 1997. NOTE: Mostly, especially in earlier literature, reported as s. lat. and should be rejected as Anthelia juratzkana. Only possibly reliable report is by Rejment-Grochowska (1967; cf. Frisvoll \& Elvebakk, 1996).

Barbilophozia Loeske

*** Barbilophozia lycopodioides (Wallr.) Loeske [Jungermannia lycopodioides Wallr., Lophozia lycopodioides (Wallr.) Cogn.]. SVALBARD: Berggren 1875b, Schiffner 1898, Frye \& Clark 1945, Schuster 1969, Worley 1972, Düll 1983, Söderström 1995, Frisvoll \& Elvebakk 1996, Paton 1999, Damsholt 2002, Söderström et al. 2002a, Damsholt 2013, Hodgetts 2015, Hodgetts \& Lockhart 2020. BJøRNØYA: Berggren 1875a, Söderström 1995, Frisvoll \& Elvebakk 1996 [doubting report], Damsholt 2002. GuSTAV V LAND: Berggren 1875a [rejected as $B$. hatcheri by Arnell \& Mårtensson 1959]. AlBERT I LAND: Lindberg 1866, Berggren 1875a [rejected as Barbilophozia hatcheri by Arnell \& Mårtensson 1959]. HAAKON VII LAND: Arnell \& Mårtensson 1959, Wegener et al. 1992. №RDENSKIÖLD LAND: Berggren 1875a, Hagen 1952, Prestø et al. 2014. OSCAR II LAND: Watson 1922 as Lophozia lycopodioides var. obliqua. NOTE: Many, especially older, reports refer to Barbilophozia hatcheri (cf. Frisvoll \& Elvebakk, 1996). We are not aware of any confirmed report from Svalbard although several specimens in TRH is named Barbilophozia lycopodioides, but they are in need of revision. Specimens in TRH that we have seen seem to deviate morphologically from what is found in boreal and alpine areas of Scandinavia. Further studies are needed to clarify the taxonomic status of these specimens.

Blepharostoma (Dumort.) Dumort.

*** Blepharostoma trichophyllum (L.) Dumort. [Jungermannia trichophylla L.]. SvalBARD: Berggren 1875b, Müller 1914a, Arnell 1928, Harmsen \& Seidenfaden 1932, Frye \& Clark 1943, Le Gallo 1951, Worley 1972, Miller et al. 1983, Smith 1990, Söderström 1995, Paton 1999, Damsholt 2002, Söderström et al. 2002a, Söderström et al. 2002b, Hodgetts 2015, Bakalin et al. 2020c, Hodgetts \& Lockhart 2020. BıøRnøYA: Watson 1922, Summerhayes \& Elton 1923a, Söderström 1995, Damsholt 2002, Söderström et al. 2002b. GustaV V LAND: Wulff 1902. EDGEøYa: Heinemeijer 1979. KONG KaRLS LAND: Arnell 1900. Lindberg 1866 [Isfjorden], Nilsen \& Elvebakk 2014 ["Sassendalen"]. ALBERT I LAND: Gottsche et al. 1847, Berggren 1875a, Frisvoll 1978. BüNSOW LAND: Watson 1922, Summerhayes \& Elton 1923a, Acock 1940. DICKSON LAND: Frisvoll 1981. HAAKON VII LAND: Frisvoll 1978, Wegener et al. 1992, Newsham \& GoodallCopestake 2021. NORDENSKIÖLD LAND: Watson 1922, Summerhayes \& Elton 1923b, Hadač 1946, Hagen 1952, Eurola 1968, Hadač 1989, Konstantinova \& Savchenko 2007 [s.lat.], Virtanen et al. 2013, Prestø et al. 2014. NyFRIESLAND: Wulff 1902. OSSCAR II LAND: Watson 1922, Summerhayes \& Elton 1923a, 1923b, Brattbakk et al. 1978, Gugnacka-Fiedor \& Nryśkiewicz 1982, Boińska \& Gugnacka-Fiedor 1983, Kobayashi et al. 1990, Koroleva \& Borovichev 2014, Newsham \& Goodall-Copestake 2021. PRINS KaRlS Forland: Hagen 1908. S. ØrKaPP LAND: Dubiel \& Olech 1990. WeDEL JARLSBERG LAND: Karczmarz \& Święs 1990a, 1990b, Święs \& Karczmarz 1991a, 1991b, 1993b, Kuc 1998. NOTE: Only reported s.lat. and probably only Blepharostoma brevirete occurs on Svalbard.

\section{Harpanthus Nees}

*** Harpanthus flotovianus (Nees) Nees. SvaLBARD: Müller 1911, Arnell 1928 ['flotowianus'], Jørgensen 1934 as Harpanthus flotovianus var. retusa ['flotowianus'], Frye \& Clark 1945, Arnell 1956 ['flotowianus'], Grolle 1965, Worley 1972, Steere \& Inoue 1978, Schljakov 1979, Schuster 1980, Smith 1990, Söderström 1995, Paton 1999, Damsholt 2002, Söderström et al. 2002a, Söderström et al. 2002b, Konstantinova 2003, Damsholt 2013, Hodgetts 2015, Hodgetts \& Lockhart 2020. KONG_KaRLS LAND: Arnell 1900, Frisvoll \& Blom 1992, Frisvoll \& Elvebakk 1996, Frisvoll \& Blom 1997. SPITSBERGEN: Wulff 1902 as Harpanthus Flotowii [Wijde bay], Frisvoll \& Blom 1992 [Wijdefjorden], Frisvoll \& Elvebakk 1996 [Wijdefjorden]. NOTE: The taxon is not seen after 1902 but we have not seen the specimens that the reports are based on. Jungermannia $\mathbf{L}$.

*** Jungermannia borealis Damsh. et Váňa. SVALBARD: Schuster 1969 as Solenostoma oblongifolium, Düll 1983, Söderström et al. 2002a, Ellis et al. 2013b, Hodgetts 2015, Hodgetts \& Lockhart 2020 [with doubt]. GuSTAV V LAND: Konstantinova \& Savchenko 2012 as Jungermannia cf. borealis. NNORDENSKIÖLD_LAND: Arnell \& Mårtensson 1959 as Jungermannia oblongifolia. NOTE: Arnell \& Mårtensson's report was rejected as Jungermannia polaris by Váňa (1973) and later only reported with some hesitation.

\section{Lophoziopsis Konstant. et Vilnet}

** Lophoziopsis longidens (Lindb.) Konstant. et Vilnet subsp. arctica (R.M.Schust.) Váňa et L.Söderstr. [Lophozia longidens subsp. arctica R.M.Schust.]. SVALBARD: Düll 1983 [with a '?'], Söderström et al. 2002a [with a '?'], Damsholt 2013, Hodgetts 2015 [with a '?'], Hodgetts \& Lockhart 2020 [with a '?']. NOTE: Lophoziopsis longidens subsp. arctica has never been confirmed for Svalbard and the existing secondary reports seem to be based only on the possibility that the subspecies occurs there. However, there is a specimen in TRH identified by A.A. Frisvoll as this subspecies, but the specimen is not available to re-study at the moment.

Tritomaria Schiffn. ex Loeske

** Tritomaria exsectiformis (Breidl.) Schiffn. ex Loeske subsp. arctica R.M.Schust. S valbarD: Söderström 1995, Damsholt 2002, 2013, Söderström et al. 2002b, Hodgetts 2015, Hodgetts \& Lockhart 2020. NOTE: Subspecies arcti$c a$ has not been confirmed to occur on Svalbard.

\section{TAXA REJECTED FROM SVALBARD}

\section{Barbilophozia Loeske}

*** Barbilophozia rubescens (R.M.Schust. et Damsh.) Kartt. et L.Söderstr. OSCAR II LAND: Arnell \& Mårtensson 1959 as Barbilophozia hatcheri var. grandiretis ['approaching var. grandiretis']. NOTE: Only reported with doubt and the report is rejected by Frisvoll \& Elvebakk (1996).

\section{Cephaloziella (Spruce) Schiffn.}

*** Cephaloziella divaricata (Sm.) Schiffn. [Jungermannia divaricata Sm.]. SvalBARD: Harmsen \& Seidenfaden 1932 as Cephaloziella 'starkii', Frye \& Clark 1945 as Cephaloziella byssacea, Schuster 1980 as Cephaloziella byssacea var. byssacea, Düll 1983, Smith 1990, Konstantinova 2003, Damsholt 2013. BJøRnøYA: Summerhayes \& Elton 1923a as Cephaloziella byssacea. SPITSBERGEN: Armitage 1937 as Cephaloziella 'Starkii' ["King's Heaven"]. AlbBERT I LAND: Gottsche et al. 1847. PRINS KARLS ForLAND: Watson 1922 as Cephaloziella byssacea, Summerhayes \& EIton 1923a, 1923b both as Cephaloziella byssacea. NOTE: Cephaloziel- 
la divaricata has been reported from Svalbard many times, but they are all rejected by Frisvoll \& Elvebakk (1996) as probably mostly Cephaloziella uncinata.

*** Cephaloziella grimsulana (J.B.Jack ex Gottsche et Rabenh.) Lacout. SVALBARD: Frye \& Clark 1945, Schuster 1980, Düll 1983, Damsholt 2002 [with a “?”], Damsholt 2013. Wedel Jarlsberg Land: Karczmarz \& Święs 1990b, Święs \& Karczmarz 1991a, 1991b, 1993b. NOTE: All reports was rejected by Frisvoll \& Elvebakk (1996).

*** Cephaloziella hampeana (Nees) Schiffn. ex Loeske. SVALBARD: Herzog 1926 as Cephaloziella biloba, Frye \& Clark 1945 as Cephaloziella biloba. NoRDENSKIÖLD LAND: Müller 1913a as Cephaloziella biloba. NOTE: Müller's report of Cephaloziella biloba (=Cephaloziella hampeana) was apparently overlooked by Frisvoll \& Elvebakk (1996) but the occurrence must be rejected as was done in Hodgetts (2015). *** Cephaloziella rubella (Nees) Warnst. Svalbard: Hodgetts 2015, Stebel et al. 2018,. Kong KarLS Land: Arnell 1900 as Cephalozia bifida. AlbBert I LAND: Wulff 1902 as Cephalozia bifida. NORDENSKIÖLD LAND: Ellis et al. 2017 [rejected by Konstantinova in litt. 2019-01-25]. OSCAR II LAND: Summerhayes \& EIton 1923a, 1923b both as Cephaloziella bifida var. bifida and var. erosa. NOTE: All reports were rejected by Frisvoll \& Elvebakk (1996). The "new" record in Ellis et al (2017) is also a mis-identification (re-examined by N. Konstantinova).

*** Cephaloziella spinigera (Lindb.) Warnst. SvalBARD: HAAKON VII LAND: Thingsgaard \& Damsholt 2007. NORDENSKiöld LAND: Persson 1942 as Cephaloziella striatula, Hadač 1946 as Cephalozia striatula. OSCAR II LAND: Arnell \& Mårtensson 1959 as Cephaloziella subdentata [rejected by Schuster 1980 as mostly C. uncinata]. WeDEL JARLSBERG LAND: Rejment-Grochowska 1967 as Cephaloziella subdentata. NOTE: All reports of Cephaloziella spinigera was rejected by Frisvoll \& Elvebakk (1996).

*** Cephaloziella stellulifera (Taylor ex Carrington et Pearson) Croz. Svalbard: Smith 1990, Schäfer-Verwimp \& Giancotti 1993, Damsholt 2002 [“doubtfully"]. NOTE: There seems not to be any primary report and all other reports must be rejected as done by Söderström et al. (2007).

*** Cephaloziella verrucosa Steph. Svalbard: Steere 1954. NoRDENSKIÖLD LAND: Bryhn 1909. NOTE: The report of Cephaloziella verrucosa, a Southern Hemisphere species, is probably an error of synonymy.

Chiloscyphus Corda NOTE: All reports of Chiloscyphus (under various names) probably originate from a single specimen said to be collected by Vahl in Bellsund, but this is probably an error in labelling as the expedition also visited northern Norway (cf. Frisvoll \& Elvebakk, 1996).

*** Chiloscyphus pallescens (Ehrh. ex Hoffm.) Dumort. [Chiloscyphus polyanthos var. pallescens (Ehrh. ex Hoffm.) Hartm.]. SVALBARD: Arnell 1956, Worley 1972, Smith 1990, Damsholt 2002 [with a "?"],. SPITSBERGEN: Gottsche et al. 1847.

*** Chiloscyphus polyanthos (L.) Corda. Svalbard: Arnell 1928, Frye \& Clark 1943, Le Gallo 1951, Arnell 1956, Schuster 1980, Smith 1990. SPITSBERGEN: Berggren 1875a, Schiffner 1912 as Chiloscyphus polyanthos f. luxurians.

Eremonotus Lindb. et Kaal. ex Pearson

*** Eremonotus myriocarpus (Carrington) Lindb. et Kaal. ex Pearson. Svalbard: Düll 1983, Paton 1999. NOTE: The reference to Svalbard in Düll (1983) is an error (cf. Frisvoll \& Elvebakk 1996).

\section{Fuscocephaloziopsis Fulford}

*** Fuscocephaloziopsis catenulata (Huebener) Váňa et L.Söderstr. [Cephalozia catenulata (Huebener) Lindb.]. SVALBARD: Frye \& Clark 1945, Le Gallo 1951, Schuster 1974 [with doubt]. BృøRnøYa: Watson 1922 as Cephalozia reclusa, Summerhayes \& Elton 1923a as Cephalozia serriflora, Söderström 1995. NOTE: All reports was rejected by Frisvoll \& Elvebakk (1996).

*** Fuscocephaloziopsis leucantha (Spruce) Váňa et L.Söderstr. [Cephalozia leucantha Spruce]. SVALBARD: Frye \& Clark 1945, BıøRnøya: Watson 1922 as Cephalozia leucantha, Summerhayes \& EIton 1923a. NORDENSKIÖLD LAND: Eurola 1971 as Cephalozia cf. leucantha. NOTE: All reports of Fuscocephaloziopsis leucantha was rejected by Frisvoll \& Elvebakk (1996).

\section{Gymnocolea (Dumort.) Dumort.}

* Gymnocolea inflata (Huds.) Dumort. subsp. acutiloba (Schiffn.) R.M.Schust. et Damsh. ex L.Söderstr. et Váňa [Gymnocolea acutiloba (Schiffn.) Müll.Frib.]. SvalBARD: Düll 1983. NOTE: Gymnocolea inflata subsp. acutiloba is a problematic taxon which sometimes has been treated as conspecific with Gymnocolea inflata, sometimes as a separate species. Düll's (1983) report of Gymnocolea acutiloba was regarded as an error by Frisvoll \& Elvebakk (1996).

Harpanthus Nees

*** Harpanthus scutatus (F.Weber et D.Mohr) Spruce. SvalBARD: Frye \& Clark 1945, Le Gallo 1951, Grolle 1965, Schuster 1980 [with doubt], Söderström 1995, Paton 1999, Damsholt 2002, Söderström et al. 2002a, Söderström et al. 2002b, Hodgetts 2015, Hodgetts \& Lockhart 2020 [rejecting earlier reports]. BJøRNøYA: Watson 1922, Summerhayes \& Elton 1923a, Söderström 1995, Frisvoll \& Elvebakk 1996, Frisvoll \& Blom 1997, Damsholt 2002, Söderström et al. 2002b. OSCAR II LAND: Watson 1922, Frisvoll \& Blom 1997. PrINS KARLS FoRLAND: Watson 1922, Frisvoll \& Elvebakk 1996, Frisvoll \& Blom 1997. NOTE: Both reports were considered doubtful by Frisvoll \& Elvebakk (1996) and we here exclude this mainly boreal species as highly unlikely to occur on Svalbard.

Heterogemma (Jørg.) Konstant. et Vilnet

*** Heterogemma capitata (Hook.) Konstant. et Vilnet. [Lophozia capitata (Hook.) Macoun]. S SVALBARD: Söderström et al. 2002a. NOTE: Heterogemma capitata was excluded from Svalbard by Söderström et al. (2002). However, it seems never have been reported to occur there so the exclusion was, although correct, an error.

\section{Hygrobiella Spruce}

*** Hygrobiella laxifolia (Hook.) Spruce. SValbard: Herzog 1926. NOTE: Although mentioned under the heading "Spitzbergen" it is not clear if Herzog actually meant that Hygrobiella laxifolia occurred there. However, it is never found in the Arctic and thus rejected here.

\section{Jungermannia $\mathbf{L}$.}

*** Jungermannia atrovirens Dumort. [Aplozia atrovirens (Dumort.) Dumort. 'Haplozia', Solenostoma atrovirens (Dumort.) Müll.Frib. hom. illeg.]. Svalbard: Stephani 1901b, Müller 1909c, Lorenz 1915, Frye \& Clark 1943, Amakawa 1960, Schuster 1969, Worley 1972, Smith 1990. BARENTSøYA: Philippi 1973. EDGEøYa: Philippi 1973 as Solenostoma atrovirens. NoRdENSKIÖLD LAND: Persson 1942. S SABINE LAND: Philippi 1973. NOTE: Rejected as Jungermannia polaris by Váňa (1973). 
** Jungermannia eucordifolia Schljakov. SVALBARD: Düll 1983 as Jungermannia exsertifolia. NOTE: Düll's (1983) report is erroneous (cf. Frisvoll \& Elvebakk, 1996).

Lophozia (Dumort.) Dumort.

*** Lophozia guttulata (Lindb. et Arnell) A.Evans. SvaLBARD: Frye \& Clark 1945 as Lophozia porphyroleuca, Smith 1990 as Lophozia longiflora. BJøRNøYA: Watson 1922 as Lophozia porphyroleuca, Summerhayes \& EIton 1923a as Lophozia porphyroleuca. NOTE: The occurrence of this almost strictly xylicolous species is highly unlikely and Frisvoll \& Elvebakk (1996) rejects it. Some reports are probably due to nomenclature confusions (cf. Söderström et al., 2013).

* Lophozia schusteriana Schljakov. S SvalBarD: Schuster 1969 as Lophozia groenlandica. HAAKON VII LAND: Arnell \& Mårtensson 1959 as Lophozia groenlandica. OSCAR_II LAND: Arnell \& Mårtensson 1959 as Lophozia groenlandi$c a$. NOTE: The name Lophozia groenlandica is in a few cases connected with Lophozia schusteriana but this should probably not be the case of any European material, which instead should go to Lophozia murmanica or Lophozia wenzelii.

Lophoziopsis Konstant. et Vilnet

* Lophoziopsis propagulifera (Gottsche) Konstant. et Vilnet. SvalbarD: Hodgetts 2015, Stotler \& Crandall-Stotler 2017, Ellis et al. 2019b. GuSTAV V LAND: Konstantinova \& Savchenko 2012. NOTE: The Southern Hemisphere Lophozia propagulifera has been synonymized with Lophoziopsis latifolia (=Lophoziopsis jurensis) for some time, but this is rejected by Hodgetts et al. (2020).

\section{Mannia Corda}

*** Mannia pilosa (Hornem.) Frye et L.Clark [Duvalia pilosa (Hornem.) Lindb., Grimaldia pilosa (Hornem.) Lindb., Neesiella pilosa (Hornem.) Schiffn.]. S Svalbard: Kaalaas 1893a, Schiffner 1893, Bryhn 1906, Evans 1911, Jørgensen 1934, Müller 1952, Düll 1983 [with a '?'], Schuster 1992. GuSTAV V LAND: Berggren 1875a. SPITSBERGEN: Müller 1907b [Nordfjorden]. WEDEL JARLSBERG LAND: Święs \& Karczmarz 1993b. NOTE: Mannia pilosa was rejected from Svalbard by Frisvoll \& Elvebakk (1996).

Marsupella Dumort.

*** Marsupella sphacelata (Giesecke ex Lindenb.) Dumort. SVALBARD: Mohr 1901. NOTE: The mention in a list of Alabama bryophytes is certainly an error.

Mesoptychia (Lindb.) A.Evans

*** Mesoptychia bantriensis (Hook.) L.Söderstr. et Váňa [Lophozia bantriensis (Hook.) Steph., Leiocolea bantriensis (Hook.) Jørg.]. S SvalbarD: Müller 1954b, Arnell 1956, Schuster 1969, Schuster 1981, Smith 1990. NOTE: There seems not to be any primary report and Frisvoll \& Elvebakk (1996) rejected it.

\section{Mylia Gray}

*** Mylia anomala (Hook.) Gray. SVALBARD: Müller 1956a, Smith 1990. NOTE: There seems not to exist any primary report and Frisvoll \& Elvebakk (1996) rejected the occurrence.

Nardia Gray

*** Nardia breidleri (Limpr.) Lindb. Svalbard: Konstantinova 2000, Söderström et al. 2002a, Hodgetts 2015. NOTE: Nardia breidleri is only reported from Svalbard on a map without supporting data, but this is an error for Jan Mayen.

*** Nardia compressa (Hook.) Gray. NOTE: Nardia compressa was rejected from Svalbard by Hodgetts (2015; also
Hodgetts \& Lockhardt 2020) but we are not aware of any reports at all.

*** Nardia scalaris Gray [Jungermannia scalaris (Gray) Hook., Alicularia scalaris (Gray) Corda]. S SvalbarD: Müller 1909c, Damsholt 2002, 2013, Hodgetts 2015. GusTAV V LAND: Hooker 1828. BüNSOW LAND: Acock 1940. NOTE: Old reports are unreliable and it was rejected by Frisvoll \& Elvebakk (1996).

Neoorthocaulis L.Söderstr., De Roo et Hedd.

*** Neoorthocaulis attenuatus (Mårt.) L.Söderstr., De Roo et Hedd. [Jungermannia attenuata (Mårt.) Lindenb., Lophozia attenuata (Mårt.) Dumort.]. S SvalBARD: Berggren 1875b, Kaalaas 1893c as Jungermannia gracilis, Harmsen \& Seidenfaden 1932, Frye \& Clark 1945 as Orthocaulis gracilis, Schuster 1969, Worley 1972. BJøRNøYA: Berggren 1875a. GuSTAV V LAND: Wulff 1902 as Jungermannia gracilis. $\underline{\text { KONG }}$ KARLS LAND: Arnell 1900 as Jungermannia gracilis. ALBERT I LAND: Berggren 1875a. №RDENSKIÖLD LAND: Hagen 1952. PRINS KaRLS Forland: Watson 1922, Summerhayes \& Elton 1923a, 1923b. NOTE: A mainly boreal species and the many reports from Svalbard were all rejected by Frisvoll \& Elvebakk (1996). The varieties described from Svalbard must belong to other species.

*** Neoorthocaulis binsteadii (Kaal.) L.Söderstr., De Roo et Hedd. [Barbilophozia binsteadii (Kaal.) Loeske, Lophozia binsteadii (Kaal.) A.Evans, Orthocaulis binsteadii (Kaal.) H.Buch]. SvalBARD: Damsholt 2002, Söderström et al. 2002a, Konstantinova 2003, Ellis et al. 2012, Damsholt 2013, Hodgetts 2015. EDGEøYA: Heinemeijer 1979 as Orthocaulis cf. binsteadii. WEDEL_JARLSBERG_LAND: Konstantinova \& Koroleva 2003. NOTE: Frisvoll \& Elvebakk (1996) rejected it as all specimens they had seen could be referred to other species, mostly Barbilophozia hatcheri.

*** Neoorthocaulis floerkei (F.Weber et D.Mohr) L.Söderstr., De Roo et Hedd. [Jungermannia floerkei F.Weber et D.Mohr, Barbilophozia floerkei (F.Weber et D.Mohr) Loeske, Lophozia floerkei (F.Weber et D.Mohr) Schiffn., Orthocaulis floerkei (F.Weber et D.Mohr) H.Buch]. S VALBARD: Berggren 1875b, Bryhn 1906, Jørgensen 1934, Frye \& Clark 1945, Gradstein \& Váňa 1987, Ellis et al. 2017. BJøRNØYA: Watson 1922. GUSTAV V LAND: Berggren 1875a. KONG Karls Land: Arnell 1900. Allbert I LAND: Berggren 1875a, Arnell \& Mårtensson 1959. HAAKON VII LAND: Arnell \& Mårtensson 1959. NORDENSKIÖLD LAND: Ellis et al. 2016b. Olav V Land: Berggren 1875a. OSCAR II LAND: Gugnacka-Fiedor \& Nryśkiewicz 1982, Boińska \& Gugnacka-Fiedor 1983, Virtanen et al. 2013. PRINS KARLS FORLAND: Arnell 1900, Hagen 1908. NOTE: All reports of were rejected as other species by Frisvoll \& Elvebakk (1996), many as Barbilophozia hatcheri and Tritomaria quinquedentata The reports from Nordenskiöld Land and Oscar II Land are here rejected as Neoorthocaulis hyperboreus (re-identified by N. Konstantinova).

Obtusifolium S.W.Arnell

*** Obtusifolium obtusum (Lindb.) S.W.Arnell [Lophozia obtusa (Lindb.) A.Evans]. S Svalbard: Bisang 1991, Söderström 1995, Bisang 1999, Paton 1999, Damsholt 2002. NOTE: There seems not to exist any primary report and the report was considered an error by Frisvoll \& Elvebakk (1996). Odontoschisma (Dumort.) Dumort.

*** Odontoschisma denudatum (Mårt.) Dumort. WEDEL JARLSBERG LAND: Święs \& Karczmarz 1991b. NOTE: Rejected by Frisvoll \& Elvebakk (1996). 
*** Odontoschisma sphagni (Dicks.) Dumort. OSCAR II LAND: Summerhayes \& Elton 1923a, 1923b, Ellis et al. 2016a. NOTE: Rejected by Frisvoll \& Elvebakk (1996). The report in Ellis et al. (2016a) is also certainly wrong.

\section{Orthocaulis H.Buch}

* Orthocaulis cavifolius H.Buch et S.W.Arnell [Sphenolobus cavifolius (H.Buch et S.W.Arnell) Müll.Frib.]. SvaLBARD: Düll 1983 [with a '?'], Konstantinova \& Savchenko 2020b. HeER LAND: Philippi 1973. NOTE: Rejected as Sphenolobus minutus by Frisvoll \& Elvebakk (1996).

\section{Pellia Raddi}

*** Pellia neesiana (Gottsche) Limpr. SvalbarD: Lindberg \& Arnell 1889 as Marsilea neesii. NOTE: This old report must be rejected.

Plagiochila (Dumort.) Dumort.

*** Plagiochila porelloides (Torr. ex Nees) Lindenb. SvaLBARD: Düll 1983. NOTE Rejected as an error by Frisvoll \& Elvebakk (1996).

*** Plagiochila spinulosa (Dicks.) Dumort. S SvalbarD: Phips 1774 as Lichenastrum ramosius, foliis trifidis. NOTE: Only mentioned through a doubtful indirect synonym and rejected by Frisvoll \& Elvebakk (1996).

Pleurozia Dumort.

*** Pleurozia purpurea Lindb. NOTE: Rejected from Svalbard by Söderström et al. (2007) as it has only been reported from Jan Mayen, and the occurrence there is also highly questionable.

Protolophozia (R.M.Schust.) Schljakov

*** Protolophozia elongata (Steph.) Schljakov [Lophozia elongata Steph., Orthocaulis elongatus (Steph.) A.Evans]. SVALBARD: Herzog 1926, Ladyzhenskaja \& Zhukova 1972, Lammes 1977, Düll 1983, Hodgetts 2015. NoRDENSKIÖLD LAND: Arnell \& Mårtensson 1959. Wedel JaRLSBERG LAND: Rejment-Grochowska 1967. NOTE: Rejected by Frisvoll \& Elvebakk (1996).

Ptilidium Nees

*** Ptilidium pulcherrimum (Weber) Vain. SVALbard: Frye \& Clark 1943, Söderström 1995. HaAkon VII Land: Polunin 1945. NORDENSKIÖLD LAND: Hagen 1952. P RINS KARLS ForLAND: Watson 1922. NOTE: Rejected by Frisvoll \& Elvebakk (1996).

Riccia $\mathbf{L}$.

*** Riccia sorocarpa Bisch. Svalbard: Müller 1952, Smith 1990, Damsholt 2002 [with a “?”], Damsholt 2013. NOTE: It is unclear what Müller based his report on and Söderström et al. (2007) rejected it.

Scapania (Dumort.) Dumort.

*** Scapania aspera M.Bernet et Bernet. Svalbard: Smith 1990. NOTE: We do not know what Smith based his reference on, but Scapania aspera do not occur on Svalbard.

*** Scapania calcicola (Arnell et J.Perss.) Ingham. SvaLBARD: Schuster 1974, Schljakov 1981, Düll 1983 [with a '?'], Smith 1990, Söderström 1995, Paton 1999, Damsholt 2002, Söderström et al. 2002a, Söderström et al. 2002b [with a '?'], Hodgetts 2015, Hodgetts \& Lockhart 2020, Unan et al. 2021. DICKSON LAND: Konstantinova \& Savchenko 2008c. HAAKON VII LAND: Arnell \& Mårtensson 1959, Frisvoll \& Elvebakk 1996. JAMES L LAND: Frisvoll \& Blom 1992. OSCAR II LAND: Arnell \& Mårtensson 1959, Frisvoll \& Elvebakk 1996, Frisvoll \& Blom 1997. NOTE: All reports are s.lat. (including "subsp. ligulifolia") and is here rejected as Scapa- nia ligulifolia as only the latter is reported s.str. from the Arctic.

*** Scapania helvetica Gottsche. OSCAR II LAND: Watson 1922 as Scapania curta var. geniculata. NOTE: Rejected by Frisvoll \& Elvebakk (1996).

*** Scapania lingulata H.Buch. NOTE: Rejected from Svalbard by Söderström et al. (2002) but there seems not to be any earlier report at all to reject.

*** Scapania mucronata H.Buch. SVALBARD: Düll 1983, Söderström 1995, Frisvoll \& Elvebakk 1996, Söderström et al. 2002a, Konstantinova 2003, Hodgetts 2015, Hodgetts \& Lockhart 2020. NORDENSKIÖLD LAND: Hagen 1952, Hadač 1989, Frisvoll \& Blom 1997. OSCAR II LAND: Frisvoll \& Blom 1997. NOTE: All reports are s. lat. or with doubt and we assume all refer to Scapania praetervisa or the very variable Scapania obcordata.

*** Scapania nemorea (L.) Grolle. S SvalbarD: Berggren 1875 b as Scapania nemorosa. AlbERT I LAND: Livesay 1870 as Jungermannia nemorosa, Berggren 1875a as Scapania nemorosa. NOTE: The old reports are rejected by Frisvoll \& Elvebakk (1996).

*** Scapania scandica (Arnell et H.Buch) Macvicar. SvaLBARD: Schljakov 1981, Söderström 1995, Damsholt 2002, Konstantinova 2003. WEDEL JARLSBERG LAND: Święs \& Karczmarz 1991a. NOTE: Only reported s.lat. and we think all belong to Scapania parvifolia.

*** Scapania undulata (L.) Dumort. Svalbard: Berggren 1875b, Müller 1901, 1915a, Bryhn 1906, Jørgensen 1934, Smith 1990. BJøRNøYA: Berggren 1875a. GUSTAV V LAND: Berggren 1875a as Scapania nemorosa var. purpurascens. ALBERT I LAND: Berggren 1875a also as Scapania undulata var. integrifolia and Scapania nemorosa var. purpurascens. OSCAR II LAND: Newsham \& Goodall-Copestake 2021. NOTE: Rejected as partly Scapania tundrae and partly Scapania hyperborea by Frisvoll \& Elvebakk (1996).

Solenostoma Mitt.

*** Solenostoma gracillimum (Sm.) R.M.Schust. [Jungermannia gracillima Sm.]. Svalbard: Buch \& Fagerström [rejecting earlier reports], Váňa 1974. NORDENSKIÖLD_LAND: Berggren 1875a as Jungermannia genthiana. WEDEL JARLSBERG_LAND: Święs \& Karczmarz 1991b as Solenostoma crenulatum var. nana. NOTE: Rejected from Svalbard already by Buch \& Fagerström (1946; see also Frisvoll \& Elvebakk, 1976).

\section{NAMES WITH UNCLEAR AFFINITIES}

Jungermannia alpestris Schleich. ex F.Weber NOTE: Jungermannia alpestris was used in the sense of Barbilophozia sudetica by Lindberg, but the identity of the two infrataxa is unknown to us. $-\alpha * * * *$ julacea Nees. AlBERT I LAND: Lindberg 1866. $-\gamma *$ subintegra Nees. ALBERT I LAND: Lindberg 1866.

Lophozia attenuata (Mårt.) Dumort. f. biloba A.Hagen, nom. inval. NORDENSKIÖLD LAND: Original material, Hagen 1952. NOTE: Lophozia attenuata is now Neoorthocaulis attenuatus, a species excluded from Svalbard. However, the identity of the specimen named f. biloba by Hagen is unknown to us.

Scapania undulata B $\beta 1$ gemmipara Lindb. ALBERT I LAND: TYPE, Lindberg 1866. NOTE: Scapania undulata is rejected from Svalbard but the identity of Lindberg's taxon is unknown to us. 


\section{SYNONYMS}

Alicularia scalaris (Gray) Corda $\equiv$ Nardia scalaris Anastrophyllum cavifolium (H.Buch et S.W.Arnell) Lammes $\equiv$ Orthocaulis cavifolius

A. minutum (Schreb.) R.M.Schust. $\equiv$ Sphenolobus minutus

A. minutum var. weberi (Mårt.) Kartt. = Sphenolobus minutus

A. sphenoloboides R.M.Schust. $\equiv$ Schizophyllopsis sphenoloboides

Aneura pinguis $\alpha 2$ minor Nees $=$ Aneura pinguis

A. pinguis $\alpha 3$ tenuis Nees $=$ Aneura pinguis

Aplozia atrovirens (Dumort.) Dumort. 三 Jungermannia atrovirens

A. polaris (Lindb.) Bryhn et Kaal. 三 Jungermannia polaris

Athalamia hyalina (Sommerf.) S.Hatt. $\equiv$ Clevea hyalina

Barbilophozia attenuata (Mårt.) Loeske $\equiv$ Neoorthocaulis attenuatus

B. binsteadii (Kaal.) Loeske $\equiv$ Neoorthocaulis binsteadii

B. floerkei (F.Weber et D.Mohr) Loeske $\equiv$ Neoorthocaulis floerkei

B. hatcheri var. grandiretis H.Buch ex Lammes $=$ Barbilophozia rubescens

B. kunzeana (Huebener) Müll.Frib. = Schljakovia kunzeana

B. quadriloba (Lindb.) Loeske $\equiv$ Schljakovianthus quadrilobus

B. quadriloba var. glareosa (Jørg.) Lammes = Schljakovianthus quadrilobus

Blepharostoma setiforme (Ehrh.) Schiffn. $\equiv$ Tetralophozia setiformis

B. trichophyllum subsp. brevirete (Bryhn et Kaal.) R.M.Schust. $\equiv$ Blepharostoma brevirete

B. trichophyllum var. brevirete Bryhn et Kaal. $\equiv$ Blepharostoma brevirete

Blepharozia ciliaris (L.) Dumort. $\equiv$ Ptilidium ciliare

Bucegia romanica Radian $\equiv$ Marchantia romanica

Cephalozia bicuspidata subsp. ambigua (C.Massal.) R.M.Schust.

$\equiv$ Cephalozia ambigua

C. bifida Lindb. $=$ Cephaloziella rubella

C. catenulata (Huebener) Lindb. $\equiv$ Fuscocephaloziopsis catenulata

C. divaricata var. incurva (Lindb.) Arnell et C.E.O.Jensen nom. illeg. $=$ Cephaloziella varians

C. leucantha Spruce $\equiv$ Fuscocephaloziopsis leucantha

C. lunulifolia (Dumort.) Dumort. $\equiv$ Fuscocephaloziopsis lunulifolia

C. media Lindb. $=$ Fuscocephaloziopsis lunulifolia

C. pleniceps (Austin) Lindb. $\equiv$ Fuscocephaloziopsis pleniceps

C. reclusa (Taylor) Dumort. $=$ Fuscocephaloziopsis catenulata

C. serriflora Lindb. $=$ Fuscocephaloziopsis catenulata

C. striatula C.E.O.Jensen $=$ Cephaloziella spinigera

Cephaloziella alpina Douin $=$ Cephaloziella varians

C. arctica Bryhn et Douin = Cephaloziella varians

C. bifida var. erosa Summerh. et Elton nom. inval. $=$ Cephaloziella rubella

C. biloba (Lindb.) Müll.Frib. = Cephaloziella hampeana

C. byssacea $($ Roth) Schiffn. $=$ Cephaloziella hampeana

C. starkei (Dumort. ex Cogn.) Schiffn. = Cephaloziella divaricata

C. striatula (C.E.O.Jensen) Douin $=$ Cephaloziella spinigera

C. subdentata Warnst. = Cephaloziella spinigera

Cesius concinnatus (Lightf.) Gray $\equiv$ Gymnomitrion concinnatum

C. corallioides (Nees) Carruth. $\equiv$ Gymnomitrion corallioides

Chandonanthus setiformis (Ehrh.) Lindb. $\equiv$ Tetralophozia setiformis

C. setiformis var. alpinus (Hook.) Kaal. = Tetralophozia setiformis
Chiloscyphus polyanthos var. pallescens (Ehrh. ex Hoffm.) Hartm. $\equiv$ Chiloscyphus pallescens

Chomiocarpon commutatum (Lindenb.) Lindb. = Marchantia quadrata

C. quadratum (Scop.) Lindb. $\equiv$ Marchantia quadrata

Cladopodiella francisci (Hook.) Jørg. = Odontoschisma francisci

Clevea hyalina f. rufescens S.W.Arnell, nom. inval. $=$ Clevea hyalina

C. hyalina var. rufescens (S.W.Arnell) Konstant. nom. inval. = Clevea hyalina

Dichiton integerrimum (Lindb.) H.Buch $\equiv$ Cephaloziella integerrima

Diplophyllum taxifolium var. macrostictum H.Buch $=$ Diplophyllum taxifolium

Duvalia pilosa (Hornem.) Lindb. $\equiv$ Mannia pilosa

Eucephalozia bicuspidata (L.) Schiffn. $\equiv$ Cephalozia bicuspidata

Grimaldia pilosa (Hornem.) Lindb. $\equiv$ Mannia pilosa

Gymnocolea acutiloba (Schiffn.) Müll.Frib. = Gymnocolea inflata subsp. acutiloba

Gymnomitrion apiculatum (Schiffn.) Müll.Frib. = Marsupella apiculata

G. condensatum Ångstr. ex C.Hartm. = Marsupella condensata

Harpanthus flotovianus var. retusa Jørg. = Harpanthus flotovianus

Jungermannia acuta Lindenb. nom. illeg. = Mesoptychia collaris

J. albicans L. $\equiv$ Diplophyllum albicans

J. alpestris Schleich. nom. inval. $=$ Mesoptychia collaris

$J$. alpestris Schleich. ex F.Weber $=$ Mesoptychia collaris

$J$. alpestris auct. plur. $=$ Barbilophozia sudetica

$J$. attenuata (Mårt.) Lindenb. $\equiv$ Neoorthocaulis attenuatus

$J$. attenuata $\beta^{*}$ laxifolia Lindb. = Trilophozia quinquedentata

J. barbata Schmidel ex Schreb. $\equiv$ Barbilophozia barbata

J. bicuspidata A $\beta *$ gracillima Nees = Cephalozia ambigua

J. bicuspidata L. $\equiv$ Cephalozia bicuspidata

$J$. ciliaris $\mathrm{L} . \equiv$ Ptilidium ciliare

$J$. confertissima Nees $\equiv$ Solenostoma confertissimum

J. divaricata $\mathrm{Sm} . \equiv$ Cephaloziella divaricata

J. divaricata var. $\varepsilon$ incurva Lindb. = Cephaloziella varians

J. exsertifolia subsp. cordifolia (Dumort.) Ván̆a = Jungermannia eucordifolia

J. floerkei F.Weber et D.Mohr $\equiv$ Neoorthocaulis floerkei

J. floerkei var. arcuata (Nees) Berggr. = Barbilophozia barbata

J. floerkei F.Weber et D.Mohr ä gemmipara Lindb. $=$ Schljakovianthus quadrilobus

$J$. genthiana Huebener $=$ Solenostoma gracillimum

$J$. gracilis Schleich. nom. inval. $=$ Neoorthocaulis attenuatus

J. gracillima $\mathrm{Sm} . \equiv$ Solenostoma gracillimum

$J$. inflata Huds. $\equiv$ Gymnocolea inflata

J. inflata var. rigidiuscula Berggr. = Gymnocolea inflata

$J$. islandica Nees $\equiv$ Fuscocephaloziopsis albescens var. islandica

$J$. jenseniana (C.E.O.Jensen) Grolle $=$ Solenostoma sphaerocarpum

J. julacea L. $\equiv$ Anthelia julacea

J. julacea $\beta$ clavuligera $($ Nees) Nees $=$ Anthelia juratzkana

J. kunzei var. plicata (C.Hartm.) Lindb. nom. inval. $=$ Schljakovia kunzeana

J. longidens Lindb. $\equiv$ Lophoziopsis longidens

J. lycopodioides Wallr. $\equiv$ Barbilophozia lycopodioides

J. lycopodioides Wallr. var. cavifolia Berggr. = Barbilophozia hatcheri

J. minuta 1 fasciculata Nees $=$ Sphenolobus minutus

J. minuta Schreb. $\equiv$ Sphenolobus minutus 
J. muelleri Nees ex Lindenb. $=$ Mesoptychia collaris

J. nemorosa L. nom. illeg. = Scapania nemorea

J. oblongifolia (Müll.Frib.) H.Buch, A.Evans et Verd. nom. illeg. = Jungermannia pumila

J. obovata Nees $\equiv$ Solenostoma obovatum

J. obovata subsp. minor (Carrington) Damsh. $=$ Solenostoma obovatum

J. obovata subsp. minor (Carrington) Schljakov ex L.Söderstr. nom. inval. $=$ Solenostoma obovatum

J. plicata C.Hartm. = Schljakovia kunzeana

J. plicata var. gracilis Berggr. nom. illeg. = Schljakovia kunzeana

$J$. plicata $\gamma$ gracilis Nees $=$ Schljakovia kunzeana

J. polita Nees $\equiv$ Saccobasis polita

J. pumila * polaris (Lindb.) Berggr. $\equiv$ Jungermannia polaris

J. pumila subsp. polare (Lindb.) R.M.Schust. $\equiv$ Jungermannia polaris

$J$. pusilla (C.E.O.Jensen) H.Buch nom. illeg. $=$ Solenostoma sphaerocarpum

J. quadriloba Lindb. $\equiv$ Schljakovianthus quadrilobus

J. quinquedentata Huds. $\equiv$ Trilophozia quinquedentata

J. scalaris (Gray) Hook. nom. illeg. $=$ Nardia scalaris

J. schiffneri (Loitl.) A.Evans = Jungermannia polaris

J. setiformis Ehrh. $\equiv$ Tetralophozia setiformis

$J$ setiformis var. $\beta$ alpinus Hook. $=$ Tetralophozia setiformis

J. sphaerocarpa Hook. $\equiv$ Solenostoma sphaerocarpum

J. sphaerocarpa var. nana (Nees ex Flot.) Frye et L.Clark nom. illeg. $=$ Solenostoma sphaerocarpum

J. subelliptica $($ Lindb. ex Heeg) Levier = Solenostoma obovatum

J. taxifolia Wahlenb. $\equiv$ Diplophyllum taxifolium

J. taylorii Hook. $\equiv$ Mylia taylorii

J. tessellata (Berggr.) Berggr. $=$ Odontoschisma macounii

J. trichophylla L. $\equiv$ Blepharostoma trichophyllum

$J$. ventricosa b laxa Lindenb. = Lophozia ventricosa

$J$ ventricosa Dicks. $\equiv$ Lophozia ventricosa

$J$. ventricosa $\alpha$ * gemmipara Nees nom. illeg. = Lophozia ventri$\cos a$

J. ventricosa $\beta *$ pulchella (Nees) C.Massal. et Carestia = Lophozia ventricosa

$J$. wenzelii Nees $\equiv$ Lophozia wenzelii

Leiocolea alpestris (Schleich. ex F.Weber) Isov. nom. inval. $=$ Mesoptychia collaris

L. badensis (Gottsche ex Rabenh.) Jørg. = Mesoptychia badensis

L. badensis var. apiculata (R.M.Schust.) Konstant. et A.N.Savchenko nom. inval. $=$ Mesoptychia badensis

L. bantriensis (Hook.) Jørg. = Mesoptychia bantriensis

L. collaris (Nees) Schljakov $\equiv$ Mesoptychia collaris

L. gillmanii (Austin) A.Evans $\equiv$ Mesoptychia gillmanii

L. gillmanii f. orbiculata (R.M.Schust.) Konstant. = Mesoptychia gillmanii

L. heterocolpos (Thed. ex Hartm.) H.Buch $\equiv$ Mesoptychia heterocolpos

L. heterocolpos var. arctica (S.W.Arnell) Mårt. = Mesoptychia heterocolpos var. arctica

L. heterocolpos var. harpanthoides (Bryhn et Kaal.) R.M.Schust. ex S.W.Arnell $\equiv$ Mesoptychia heterocolpos var. harpanthoides

L. muelleri (Nees ex Lindenb.) Jørg. = Mesoptychia collaris

L. rutheana (Limpr.) Müll.Frib. = Mesoptychia rutheana

Leiomylia anomala (Hook.) J.J.Engel et Braggins $\equiv$ Mylia anomala

Lophozia alpestris (Schleich. ex F.Weber) A.Evans = Mesoptychia collaris

L. alpestris auct. plur. $=$ Barbilophozia sudetica
L. arctica (S.W.Arnell) Święs et Karczm. nom. inval. $\equiv$ Mesoptychia heterocolpos var. arctica

L. attenuata (Mårt.) Dumort. $\equiv$ Neoorthocaulis attenuatus

L. badensis (Gottsche ex Rabenh.) Schiffn. $\equiv$ Mesoptychia badensis

L. bantriensis (Hook.) Steph. $\equiv$ Mesoptychia bantriensis

L. barbata (Schmidel ex Schreb.) Dumort. 三 Barbilophozia barbata

L. bicrenata (Schmidel ex Hoffm.) Dumort. $\equiv$ Isopaches bicrenatus

L. bicrenata var. bicrenata $=$ Isopaches bicrenatus var. bicrenatus

L. binsteadii (Kaal.) A.Evans $\equiv$ Neoorthocaulis binsteadii

L. capitata (Hook.) Macoun $\equiv$ Heterogemma capitata

L. cavifolia (H.Buch et S.W.Arnell) R.M.Schust. $\equiv$ Orthocaulis cavifolius

L. collaris (Nees) Dumort. $\equiv$ Mesoptychia collaris

L. elongata Steph. $\equiv$ Protolophozia elongata

L. excisa (Dicks.) Dumort. $\equiv$ Lophoziopsis excisa

L. excisa var. cylindracea (Dumort.) Müll.Frib. = Lophoziopsis excisa var. excisa

L. excisa var. elegans R.M.Schust. $\equiv$ Lophoziopsis excisa var. elegans

L. floerkei (F.Weber et D.Mohr) Schiffn. $\equiv$ Neoorthocaulis floerkei

L. gillmanii (Austin) R.M.Schust. $\equiv$ Mesoptychia gillmanii

L. grandiretis (Lindb. ex Kaal.) Schiffn. 三 Protochilopsis grandiretis

L. grandiretis subsp. proteidea (Arnell) Damsh. $=$ Protochilopsis grandiretis

L. groenlandica $($ Nees) Macoun $=$ Lophozia murmanica

L. hatcheri (A.Evans) Steph. $\equiv$ Barbilophozia hatcheri

L. heterocolpos (Thed. ex Hartm.) M.Howe $\equiv$ Mesoptychia heterocolpos

L. heterocolpos var. arctica (S.W.Arnell) R.M.Schust. et Damsh. $\equiv$ Mesoptychia heterocolpos var. arctica

L. hyperarctica R.M.Schust. nom. inval. = Schistochilopsis hyperarctica

L. incisa (Schrad.) Dumort. $\equiv$ Schistochilopsis incisa

L. incisa subsp. opacifolia (Culm. ex Meyl.) R.M.Schust. et Damsh. nom. inval. $\equiv$ Schistochilopsis opacifolia

L. jurensis Meyl. ex Müll.Frib. = Lophoziopsis jurensis

L. kiaerii Jørg. = Lophoziopsis excisa

L. kunzeana (Huebener) A.Evans $\equiv$ Schljakovia kunzeana

L. latifolia R.M.Schust. = Lophoziopsis jurensis

L. longidens (Lindb.) Macoun $\equiv$ Lophoziopsis longidens

L. longidens subsp. arctica R.M.Schust. $\equiv$ Lophoziopsis longidens subsp. arctica

L. longiflora (Nees) Schiffn. $\equiv$ Lophozia ventricosa var. longiflora

L. lycopodioides (Wallr.) Cogn. $\equiv$ Barbilophozia lycopodioides

L. lycopodioides var. obliqua Müll.Frib. = Barbilophozia lycopodioides

L. major (C.E.O.Jensen) Schljakov = Lophoziopsis excisa var. excisa

L. muelleri (Nees ex Lindenb.) Dumort. = Mesoptychia collaris

L. obtusa (Lindb.) A.Evans $\equiv$ Obtusifolium obtusum

L. opacifolia (Culm.) Culm. ex Meyl. = Schistochilopsis opacifolia

L. pellucida R.M.Schust. nom. inval. $=$ Lophoziopsis pellucida

L. pellucida R.M.Schust. $\equiv$ Lophoziopsis pellucida

L. pellucida var. minor R.M.Schust. $\equiv$ Lophoziopsis pellucida var. minor

L. perssonii H.Buch et S.W.Arnell $\equiv$ Oleolophozia perssonii

L. polaris (R.M.Schust.) R.M.Schust. et Damsh. $\equiv$ Lophoziopsis polaris 
L. polaris var. sphagnorum (R.M.Schust.) R.M.Schust. et Damsh. $\equiv$ Lophoziopsis polaris var. sphagnorum

L. polita (Nees) Macoun $\equiv$ Saccobasis polita

L. porphyroleuca (Nees) Schiffn. nom. illeg. = Lophozia guttulata

L. quadriloba (Lindb.) A.Evans $\equiv$ Schljakovianthus quadrilobus

L. quadriloba var. glareosa (Jørg.) Jørg. = Schljakovianthus quadrilobus

L. quinquedentata (Huds.) Cogn. $\equiv$ Trilophozia quinquedentata

L. quinquedentata f. turgida (Lindb.) Summerh. et Elton = Trilophozia quinquedentata

L. quinquedentata var. turgida (Lindb.) Müll.Frib. = Trilophozia quinquedentata

L. rubescens R.M.Schust. et Damsh. $\equiv$ Barbilophozia rubescens

L. rubrigemma R.M.Schust. $\equiv$ Lophoziopsis rubrigemma

L. rufescens Schljakov $=$ Barbilophozia sudetica

L. rutheana (Limpr.) M.Howe $\equiv$ Mesoptychia rutheana

L. silvicoloides var. paroica Vilnet, Milyutina, A.V.Troitsky et Konstant. nom. inval. $=$ Lophozia silvicoloides

L. spitsbergensis Konstant. et Vilnet nom. inval. $=$ Lophozia svalbardensis

L. sudetica (Nees ex Huebener) Grolle $\equiv$ Barbilophozia sudetica

L. sudetica var. anomala (Schljakov) Schljakov = Barbilophozia sudetica

L. ventricosa var. silvicola (H.Buch) E.W.Jones $\equiv$ Lophozia silvicola

L. wenzelii var. groenlandica $(\mathrm{Nees})$ Bakalin = Lophozia murmanica

Marchantia alpestris (Nees) Burgeff = Marchantia polymorpha subsp. montivagans

M. hyalina Sommerf. $\equiv$ Clevea hyalina

M. latifolia Gray = Marchantia polymorpha subsp. ruderalis

M. polymorpha $\mathrm{B}$ alpestris Nees = Marchantia polymorpha subsp. montivagans

Marsilea neesii Lindb. $=$ Pellia neesiana

Marsupella emarginata var. arctica (Berggr.) Frye et L.Clark $\equiv$ Marsupella arctica

M. groenlandica C.E.O.Jensen $=$ Marsupella arctica

M. obcordata (Berggr.) Steph. $\equiv$ Scapania obcordata

Martinellius curtus (Mårt.) Lindb. $\equiv$ Scapania curta

M. hyperboreus (Jørg.) H.A.Möller = Scapania hyperborea

M. irriguus (Nees) Lindb. $\equiv$ Scapania irrigua

M. paludicola (Loeske et Müll.Frib.) C.E.O.Jensen = Scapania paludicola

M. spitsbergensis Lindb. $\equiv$ Scapania spitsbergensis

Massula grandiretis (Lindb. ex Kaal.) Schljakov $\equiv$ Protochilopsis grandiretis

Mesoptychia badensis var. apiculata (R.M.Schust.) Potemkin = Mesoptychia badensis

Neesiella pilosa (Hornem.) Schiffn. $\equiv$ Mannia pilosa

Orthocaulis binsteadii (Kaal.) H.Buch $\equiv$ Neoorthocaulis binsteadii

O. elongatus (Steph.) A.Evans $\equiv$ Protolophozia elongata

O. floerkei (F.Weber et D.Mohr) H.Buch $\equiv$ Neoorthocaulisfloerkei

O. gracilis (Lindenb.) H.Buch nom. illeg. $=$ Neoorthocaulis attenuatus

O. kunzeanus (Huebener) H.Buch $\equiv$ Schljakovia kunzeana

O. quadrilobus (Lindb.) H.Buch $\equiv$ Schljakovianthus quadrilobus

O. quadrilobus var. glareosa (Jørg.) S.W.Arnell nom. inval. = Schljakovianthus quadrilobus

Plectocolea obovata (Nees) Mitt. $\equiv$ Solenostoma obovatum

P. subelliptica (Lindb. ex Heeg) A.Evans = Solenostoma obovatum
Pleurocladula albescens (Hook.) Spruce $\equiv$ Fuscocephaloziopsis albescens

P. albescens (Hook.) Grolle $\equiv$ Fuscocephaloziopsis albescens

P. albescens var. islandica (Nees) L.Söderstr. et Váňa $\equiv$ Fuscocephaloziopsis albescens var. islandica

P. islandica (Nees) Grolle $\equiv$ Fuscocephaloziopsis albescens var. islandica

Preissia commutata $($ Lindenb.) Nees = Marchantia quadrata

P. quadrata (Scop.) Nees $\equiv$ Marchantia quadrata

P. quadrata subsp. hyperborea R.M.Schust. $\equiv$ Marchantia quadrata subsp. hyperborea

Pseudolophozia debiliformis (R.M.Schust. et Damsh.) Konstant. et Vilnet $=$ Barbilophozia sudetica

P. sudetica (Nees ex Huebener) Konstant. et Vilnet $\equiv$ Barbilophozia sudetica

Ptilidium ciliare f. inundatum $($ Schiffn. $)$ Macvicar $=$ Ptilidium ciliare

Riccardia pinguis (L.) Gray $\equiv$ Aneura pinguis

Sarcocyphos emarginatus var. arcticus Berggr. $\equiv$ Marsupella arctica

S. obcordatus Berggr. $\equiv$ Scapania obcordata

Sauteria hyalina (Sommerf.) Lindb. $\equiv$ Clevea hyalina

Scapania bartlingii (Hampe ex Nees) Nees = Scapania cuspiduligera var. cuspiduligera

S. bartlingii var. $\beta$ elongata Lindb. $=$ Scapania cuspiduligera

S. bartlingii var. $\gamma$ obtusata Lindb. $=$ Scapania cuspiduligera

$S$. berggrenii Warnst. $=$ Scapania tundrae

S. calcicola subsp. ligulifolia (R.M.Schust.) Damsh. et D.G.Long = Scapania ligulifolia

S. carestiae De Not. = Scapania cuspiduligera var. cuspiduligera

S. curta var. geniculata (C.Massal.) Müll.Frib. = Scapania helvetica

S. gymnostomophila f. incurva (Bryhn et Kaal.) R.M.Schust. = Scapania gymnostomophila

S. hyperborea var. tundrae (Arnell) Potemkin $\equiv$ Scapania tundrae

S. irrigua $\gamma$ globulifera (Nees) Gottsche, Lindenb. et Nees $=$ Scapania irrigua

S. mucronata subsp. praetervisa (Meyl.) R.M.Schust. $\equiv$ Scapania praetervisa

S. nemorosa (Dumort.) Dumort. nom. illeg. = Scapania nemorea

S. nemorosa var. $\beta$ purpurascens (Hook.) Gottsche, Lindenb. et Nees $=$ Scapania undulata

S. scandica f. parvifolia (Warnst.) Schljakov $\equiv$ Scapania parvifolia

S. scandica var. parvifolia (Warnst.) Konstant. et Czernjad. nom. inval. $\equiv$ Scapania parvifolia

S. undulata var. integrifolia Lindb. = Scapania undulata

Schistochilopsis grandiretis (Lindb. ex Kaal.) Konstant. $\equiv$ Protochilopsis grandiretis

S. grandiretis var. parviretis (R.M.Schust.) R.M.Schust. ex Konstant. et A.N.Savchenko nom. inval. $=$ Protochilopsis grandiretis

Schizophyllum sphenoloboides (R.M.Schust.) Váňa et L.Söderstr. $\equiv$ Schizophyllopsis sphenoloboides

Schljakovianthus quadrilobus var. glareosus (Jørg.) Konstant. et Vilnet $=$ Schljakovianthus quadrilobus

Solenostoma atrovirens (Dumort.) Müll.Frib. nom. illeg. $\equiv$ Jungermannia atrovirens

S. crenulatum var. nana Müll.Frib. = Solenostoma gracillimum

S. polare (Lindb.) R.M.Schust. $\equiv$ Jungermannia polaris

S. pumilum (With.) Müll.Frib. $\equiv$ Jungermannia pumila

S. pumilum subsp. polaris (Lindb.) R.M.Schust. $\equiv$ Jungerman- 
nia polaris

S. pusillum (C.E.O.Jensen) Steph. $=$ Solenostoma sphaerocarpum

S. sphaerocarpum var. nanum (Nees ex Flot.) Müll.Frib. $=$ Solenostoma sphaerocarpum

S. sphaerocarpum var. nanum (Nees ex Flot.) R.M.Schust. $=$ Solenostoma sphaerocarpum

S. subellipticum (Lindb. ex Heeg) R.M.Schust. = Solenostoma obovatum

Southbya fennica (Gottsche) Gottsche $\equiv$ Arnellia fennica

Sphagnoecetis communis var. tessellata Berggr. $=$ Odontoschisma macounii

Sphenolobus cavifolius (H.Buch et S.W.Arnell) Müll.Frib. $=$ Orthocaulis cavifolius

S. groenlandicus (Nees) Steph. = Lophozia murmanica

S. minutus var. grandis (Gottsche ex Lindb.) Jørg. = Sphenolobus minutus

S. politus (Nees) Steph. $\equiv$ Saccobasis polita

S. saccatulus (Lindb.) Müll.Frib. = Sphenolobus minutus

Temnoma setiforme (Ehrh.) M.Howe $\equiv$ Tetralophozia setiformis

Trilophozia quinquedentata f. gracilis (R.M.Schust.) Konstant. = Trilophozia quinquedentata

T. quinquedentata var. turgida (Lindb.) Konstant. = Trilophozia quinquedentata

Tritomaria polita (Austin) Schiffn. nom. inval. $=$ Saccobasis polita

T. polita (Nees) Jørg. = Saccobasis polita

T. polita subsp. polymorpha R.M.Schust. $\equiv$ Saccobasis polymorpha

T. quinquedentata (Huds.) H.Buch $\equiv$ Trilophozia quinquedentata

T. quinquedentata f. gracilis (C.E.O.Jensen) R.M.Schust. = Trilophozia quinquedentata

T. quinquedentata f. gracilis (C.E.O.Jensen) S.W.Arnell nom. inval. $=$ Trilophozia quinquedentata

T. quinquedentata subsp. turgida (Lindb.) Damsh. $=$ Trilophozia quinquedentata

T. quinquedentata var. turgida (Lindb.) Weim. $=$ Trilophozia quinquedentata

\section{ACKNOWLEDGEMENTS}

The project "Mapping bryophytes on Svalbard as the basis for monitoring and conservation" was funded by the Norwegian Research Council (Project number: 246728/E10), making the Norwegian-Russian collaboration possible with project meetings in Longyearbyen and Trondheim, and a week field expedition to Svalbard. The company Svalbard Explorer and the crew is thanked for making it possible to use the boat Ulla Rinman for the 2018 expedition. Xiaolan He and Sanna Laaka Lindberg, Helsinki, are thanked for the assistance in finding type material in Lindberg's herbarium in H. The work of N.A. Konstantinova was carried out partly in the framework of the State Research Program of the Polar-Alpine Botanical Garden and Institute KSC RAS (AAAA-A181021032422551-2-1.6.11).

\section{LITERATURE CITED}

ACIA. 2005. Arctic Climate Impact Assessment. Cambridge University Press, 1042 pp. ISBN 978-0-521-86509.

ACOCK, A.M. 1940. Vegetation of a calcareous inner fjord region in Spitsbergen. - Journal of Ecology 28 (1): 81-106. http://dx.doi.org/10.2307/ 2256164

AMAKAWA, T. 1960. Family Jungermanniaceae of Japan, II. - Journal of the Hattori Botanical Laboratory 22: 1-90.

ARLOV, T.B. 1994. A short history of Svalbard. - Norwegian Polar Institute, Oslo, 95 pp. ISBN 82-90307-55-1.

ARMITAGE, E. 1937. Some Spitzbergen mosses. - Bryologist 40 (4): 74-78. http://dx.doi.org/10.2307/3239120

ARNELL, H.W. 1900. Beiträge zur Moosflora der Spitzbergischen Inselgruppe. - Öfversigt af Kongliga Vetenskaps-Akademiens Förhandlingar 57: 99-130.

ARNELL, H.W. 1922. Die Schwedischen Arten der Gattungen Diplophyllum und Martinellia. - Göteborgs Kungliga Vetenskaps- och Vitterhets Samhälles handlingar (ser. 4) 26: 1-82.

ARNELL, H.W. 1928. Levermossor (in: Hartmans Handbok i Skandinaviens flora vol. 2 a edited by Otto R. Holmberg). - P. A. Norstedt \& Söner Förlag: Stockholm. 224 pp.

ARNELL, S.W. 1950. Scapania degenii and S. simmonsii found on the North American continent. - Bryologist 53 (4): 299-300. http:// dx.doi.org/10.2307/3239997

ARNELL, S.W. 1951. Blepharostoma trichophyllum (L.) Dum. var. brevirete Bryhn \& Kaal. - Botaniska Notiser 104 (1): 61-63.

ARNELL, S.W. 1956. Illustrated moss flora of Fennoscandia. I. Hepaticae. - Nordic Bryological Society: Lund. 314 pp.

ARNELL, H. W. \& C. JENSEN. 1907. Die Moose des Sarekgebietes. Erste Abteilung - In: Hamberg, A., Ed. Naturwissenschaftliche Untersuchungen des Sarekgebirges in Schwedisch-Lappland Bd. 3. Botanik. Stockholm: C. E. Fritzes, pp. 71-132.

ARNELL, S.W.\& O. MÅRTENSSON. 1959. A contribution to the knowledge of the bryophyte flora of W. Spitsbergen, and Kongsfjorden (King's Bay, $79^{\circ}$ N.) in particular. - Arkiv för Botanik (n.ser.) 4 (6): 105-164.

ARTS, T. 1986. Lophozia perssonii Buch \& S. Arnell (Hepaticae) nieuw voor België. - Dumortiera 36: 2-6.

BAKALIN, V.A. 2004. Notes on Lophozia V. Comments on sect. Sudeticae, Longidentatae and Savicziae. - Arctoa 13: 229-240. http:// dx.doi.org/10.15298/arctoa.13.16

BAKALIN, V.A. 2005. Monograph of the genus Lophozia (Dumort.) Dumort. s. str. - Nauka: Moscow. 240 pp.

BAKALIN, V.A. 2011. Notes on Lophozia VI. Taxonomy and distribution of Lophozia and Schistochilopsis (Lophoziaceae) in North America north of Mexico. - Bryologist 114 (2): 298-315. http://dx.doi.org/ 10.1639/0007-2745.114.2.298

BAKALIN, V.A. \& A.A. VILNET. 2014. Two new species of the liverwort genus Hygrobiella Spruce (Marchantiophyta) described from the North Pacific based on integrative taxonomy. - Plant Systematics and Evolution 300 (10): 2277-2291. http://dx.doi.org/10.1007/s00606014-1050-8

BAKALIN, V.A. \& A.A. VILNET. 2018. A review of the genus Diplophyllum (Marchantiophyta) in North and East Asia with the description of a new species (D. sibiricum) based on integrative taxonomy. Plant Systematics and Evolution 304 (10): 1269-1287. http:// dx.doi.org/10.1007/s00606-018-1547-7

BAKALIN, V.A. \& A.A. VILNET. 2019. Lophozia fuscovirens sp. nov. (Lophoziaceae, Marchantiophyta): the second taxon with brown gemmae within Lophozia s.s. - Nordic Journal of Botany 37 (4: e02294): 1-10. http://dx.doi.org/10.1111/njb.02294

BAKALIN, V.A, A.A. VILNET \& Y. XIONG. 2015. Mesoptychia chinensis Bakalin, Vilnet \& Xiong sp. nov. (Jungermanniaceae, Marchantiophyta) and comments on the distribution of Mesoptychia south of the boreal zone in Asia. - Journal of Bryology 37 (3): 192-201. http:/ /dx.doi.org/10.1179/1743282015Y.0000000009

BAKALIN, V.A., A.A. VILNET, K. KLIMOVA, W.Z. MA\& V.S. NGUYEN. 2020a. Diplophyllum purpurascens (Scapaniaceae, Marchantiophyta) - a new species from Sino-Himalaya (China). - Phytotaxa 447 (2): 116-126. http://dx.doi.org/10.11646/phytotaxa.447.2.4

BAKALIN, V.A., V.E. FEDOSOV, Y.D MALTSEVA, I.A. MILYUTINA, K.G. KLIMOVA, H.M. NGUYEN \& A.V. TROITSKY. 2020b. Over- 
view of Schistochilopsis (Hepaticae) in Pacific Asia with the description Protochilopsis gen. nov. - Plants 9 (7: 850): 1-33. http://dx.doi.org/ 10.3390 /plants 9070850

BAKALIN, V.A., A.A. VILNET, S.S. CHOI \& V.S. NGUYEN. 2020c. Blepharostoma trichophyllum s.1. (Marchantiophyta): The complex of sibling species and hybrids. - Plants 9 (11: 1423): 1-28. http:// dx.doi.org/10.3390/plants9111423

BAKALIN, V.A., A.A. VILNET, K, KLIMOVA, W.-Z. MA, S.-S. CHOI \& J. HENTSCHEL. 2021. Hidden in plain view: an example from Ptilidium (Ptilidiaceae, Marchantiophyta). - Phytotaxa 510 (1): 29-42. http://dx.doi.org/10.11646/PHYTOTAXA.510.1.3.

BAPNA, K. R. \& P. KACHROO. 2000a. Hepaticology in India - I. Himanshu Publications: Udaipur. 439 pp.

BAPNA, K. R. \& P. KACHROO. 2000b. Hepaticology in India - II. Himanshu Publications: Udaipur. $491 \mathrm{pp}$.

BARTHOLOMEW-BEGAN, S.E. 1991. A morphogenetic re-evaluation of Haplomitrium Nees (Hepaticopsida). - Bryophytorum Bibliotheca 41: $1-297$.

BEDNAREK-OCHYRA, H., B. GODZIK \& K. GRODZINSKA. 1987a. Bryophyta svalbardensia exsiccata, fasc. I (No. 1-20). - Polish Academy of Science: Krakow. 13 pp.

BEDNAREK-OCHYRA, H., B. GODZIK \& K. GRODZINSKA. 1987 b. Bryophyta svalbardensia exsiccata, fasc. II (No. 21-40). - Polish Academy of Science: Krakow. 13 pp.

BEDNAREK-OCHYRA, H., B. GODZIK \& K. GRODZINSKA. 1987c. Bryophyta svalbardensia exsiccata, fasc. III (No. 41-60). - Polish Academy of Science: Krakow. $11 \mathrm{pp}$.

BEDNAREK-OCHYRA, H., J. VÁŇA, R. OCHYRA \& R.I. LEWIS SMITH. 2000. The liverwort flora of Antarctica. - Polish Academy of Science: Krakow. $236 \mathrm{pp}$.

BELKINA, O.A., N.A. KONSTANTINOVA, N. KOROLEVA, L. KONOREVA, D. DAVYDOV, A. SAVCHENKO \& A. LIKHACHEV. 2015. Bryophytes, lichens and cyanoprokaryotes in surroundings of Pyramiden (Svalbard): a concise guide-book. - Russian Academy of Science, Kola Science Centre, Polar-Alpine Botanical Garden Institute: Apatity. $201 \mathrm{pp}$.

BERGGREN, S. 1875a. Musci et Hepaticae spetsbergenses. - Kongliga Svenska Vetenskaps-Akademiens Handlingar (n. ser.) 13 (7): 1-103.

BERGGREN, S. 1875b. Undersökning af mossfloran vid Disko-Bugten og Auleitsivikfjorden i Grönland. - Kungliga Svenska Vetenskapsakademiens Handlingar (n.ser.) 13 (8): 1-46.

BIRD, C.D. \& R.T. OGILVE. 1964. New bryophytes for Alberta. - Bryologist 67 (3): 360-363. http://dx.doi.org/10.2307/3240901

BISANG, I. 1991. Biosystematische Studien an Lophozia subgen. Schistochilopsis (Hepaticae). - Bryophytorum Bibliotheca 43: 1-187.

BISANG, I. 1999. Die Verbreitung von Moosen in der Schweiz und in Liechtenstein. II. Lophozia subg. Schistochilopsis. - Haussknechtia, Beiheft 9: 45-56.

BISANG I, P. GEISSLER, N. MÜLLER, N. SCHNYDER, C. SCHUBIGER-BOSSARD \& E. URMI. 1998. Die Verbreitung von Moosen in der Schweiz und in Liechtenstein [Distribution of bryophytes in Switzerland and Liechtenstein]. - Botanica Helvetica 108 (2): 197-216. http://dx.doi.org/10.5169/seals-73024

BISCHLER, H. 1984. Marchantia L. The New World species. - Bryophytorum Bibliotheca 26: 1-228.

BISCHLER-CAUSSE, H. 1989. Marchantia L. The Asiatic and Oceanic taxa. - Bryophytorum Bibliotheca 38: 1-317.

BOIŃSKA, U. \& W. GUGNACKA-FIEDOR. 1983. Bryophytes and their distribution in the tundra communities of the Kaffiöyra Plain (NW Spitsbergen). - Fragmenta Floristica et Geobotanica 29: 401-413.

BONNER, C. E. B. 1976. Index hepaticarum. Pars VIII. Jungermannia. - J. Cramer: Vaduz.

BONNER, C. E. B. 1977. Index hepaticarum. Pars VIIa. Supplementum A-C. - J. Cramer: Vaduz
BOROVICHEV, E.A. 2010. New liverwort records from Svalbard. 1. Arctoa 19: 280-281. http://dx.doi.org/10.15298/arctoa.19.24

BOROVICHEV, E.A. \& V.A. BAKALIN. 2016. On Mannia triandra (Aytoniaceae, Marchantiophyta) in eastern Asia. - Herzogia 29 (1): 59-65. http://dx.doi.org/10.13158/heia.29.1.2016.59

BOROVICHEV, E.A., V.A. BAKALIN \& A.A. VILNET. 2015. Revision of the Russian Marchantiales. II. A review of the genus Asterella P. Beauv. (Aytoniaceae, Hepaticae). - Arctoa 24 (2): 294-313. http:// dx.doi.org/10.15298/arctoa.24.26

BOROVICHEV, E.A., V. FEDOSOV A.A. VILNET. 2016. An unexpected record of the European liverwort Scapania aspera (Scapaniaceae, Marchantiophyta) in East Siberia. - Cryptogamie, Bryologie 37 (4): 445-454. http://dx.doi.org/10.7872/cryb/v37.iss4.2016.445

BRATTBAKK, I., O.I. RØNNING \& E. SENDSTAD. 1978. Økologiske studier på Svalbard. I. Økologiske effekter og nedbrytning av et simulert råoljeutslipp i strandsonen på Svalbard 1976-1977. - SINTEF Rapport STF21 A78064: $1-31$.

BRYHN, N. 1906. Bryophyta in itinere polari norvagorum secundo collecta. - Report of the Second Norwegian Arctic Expedition in the "Fram" 1898-1902 11: 1-260.

BRYHN, N. 1909. Bryophyta pro flora Spitsbergensi nova. - Nyt Magazin for Naturvidenskaberne 47 (2): 207-208.

BUCH, H. 1928. Die Scapanien Nordeuropas und Sibiriens - Systematischer Teil. - Akademische Buchhandlung: Helsingfors. 177 pp.

BUCH, H. 1933. Scapania simmonsii Bryhn et Kaal., ein für das Festland Europas neues Lebermoos. - Memoranda Societatis pro Fauna et Flora Fennica 9: 142 .

BUCH, H. \& L. FAGERSTRÖM. 1946. Scapaniella glaucocephala (Tayl.) Evans, ny för Gamla Världens flora. - Memoranda Societatis pro Fauna et Flora Fennica 22: 17-18.

BUCH, H. \& R. TUOMIKOSKI. 1955. Contribution to the hepatic flora of Newfoundland. - Archivum Societatis Zoologicae Botanicae Fennicae "Vanamo", suppl. 9: 1-29.

CRUNDWELL, A.C. 1978. Cephaloziella uncinata Schust. in Spitsbergen. - Lindbergia 4 (3/4): 297-298.

DAMSHOLT, K. 1993. Notiser on nordiska levermossor 1-5. - Svensk Botanisk Tidskrift 87: 187-191.

DAMSHOLT, K. 2002. Illustrated flora of Nordic liverworts and hornworts. - Nordic Bryological Society: Lund. 837 pp.

DAMSHOLT, K. 2003. Notes on Nordic hepatics 6-17. - Lindbergia 28 (3): $120-128$.

DAMSHOLT, K. 2013. The liverworts of Greenland. - Nordic Bryological Society: Lund. 626 pp.

DANIËLS, F.J.A, L. J. GILLESPIE \& M. POULIN. 2013. Plants. - in: Meltofte, H., Ed. Arctic Biodiversity Assessment. Status and trends in Arctic biodiversity. Conservation of Arctic Flora and Fauna, Akureyri, pp. 310-353. https://www.caff.is/assessment-series/233-arctic-biodiversity-assessment-2013

DAVISON, P.G. 1993. Floristic and phytogeographic studies of the hepatics of the Aleutian Islands. - PhD Thesis, University of Tennessee. 385 pp.

DUBIEL, E. \& M. OLECH. 1990. Plant communities of NW Sørkapp Land (Spitsbergen). - Zeszyty Naukowe Uniwersytetu Jagiellońskiego. Prace Botaniczne 21: 35-74.

DUBIEL, E. \& M. OLECH. 1992. Ornithocoprophilous plant communities on the southern slope of Ariekammen (Hornsund, Spitsbergen) in: Opalikski, K.W. \& R.Z. Klekowski, Eds. Spitsbergen '84. Landscape, life, world and man in high Arctic. Warzawa: Polish Academy of Science, pp. 167-175.

DUCKETT, J.G. \& N.G. SLACK. 2013. The first twenty-first century record for the liverwort Haplomitrium hookeri in north eastern North America with notes on its fungal endophyte and snowbed communities. - Evansia 30 (2): 53-56. http://dx.doi.org/10.1639/079.030.0203

DÜLL, R. 1983. Distribution of the European and Macaronesian liverworts (Hepaticophytina). - Bryologische Beiträge 2: 1-114. 
ELLIS, L.T., A.K. ASTHANA, V. SAHU, A. SRIVASTAVA, H. BEDNAREK-OCHYRA, R. OCHYRA, J. CHLACHULA et al. 2011. New national and regional bryophyte records, 28. - Journal of Bryology 33 (3): 237-247. http://dx.doi.org/10.1179/1743282011Y.0000000022

ELLIS, L.T., A. ALEGRO, H. BEDNAREK-OCHYRA, R. OCHYRA, A. BERGAMINI, A. COGONI, P. ERZBERGER et al. 2012. New national and regional bryophyte records, 31. - Journal of Bryology 34 (2): 123-134. http://dx.doi.org/10.1179/1743282012Y.0000000009

ELLIS, L.T., H. BEDNAREK-OCHYRA, R. OCHYRA, M.J. BENJUMEA, L.V. SAĎS, R. CAPARRÓS, F. LARA et al. 2013a. New national and regional bryophyte records, 35. - Journal of Bryology 35 (2): 129-139. http://dx.doi.org/10.1179/1743282013Y.0000000049

ELLIS, L.T., V.A BAKALIN, E. BAISHEVA, H. BEDNAREKOCHYRA, R. OCHYRA, E.A. BOROVICHEV, S.S. CHOI et al. 2013b. New national and regional bryophyte records, 36. - Journal of Bryology 35 (3): 228-238. http://dx.doi.org/10.1179/ 1743282013Y.0000000064

ELLIS, L.T., O.M. AFONINA, A.K. ASTHANA, R. GUPTA, V. SAHU, V. NATH, N. BATAN et al. 2014a. New national and regional bryophyte records, 39. - Journal of Bryology 36 (2): 134-151. http:// dx.doi.org/10.1179/1743282014Y.0000000100

ELLIS, L.T., M. ALEFFI, A.K. ASTHANA, A. SRIVASTAVA, V.A. BAKALIN, N. BATAN, T. ÖZDEMÝR et al. 2014b. New national and regional bryophyte records, 40. - Journal of Bryology 36 (3): 223244. http://dx.doi.org/10.1179/1743282014Y.0000000115

ELLIS, L.T., A. ALEGRO, V. ŠEGOTA, V.A. BAKALIN, R. BARONE, E.ABOROVICHEV, V. HUGONNOT, M. LEBOUVIER et al. 2015a. New national and regional bryophyte records, 44. - Journal of Bryology 37 (3): 228-241. http://dx.doi.org/10.1179/ 1743282015 Y.0000000014

ELLIS, L.T., C. AH-PENG, S.C. ARANDA, H. BEDNAREK-OCHYRA, E.A. BOROVICHEV, B. CYKOWSKA-MARZENCKA et al. 2015b. New national and regional bryophyte records, 45. - Journal of Bryol-

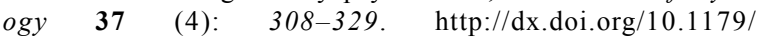
1743282015 Y.0000000035

ELLIS, L.T., M. ALEFFI, A. ALEGRO, V. SEGOTA, A.K. ASTHANA, R. GUPTA, V.J. SINGH et al. 2016A. New national and regional bryophyte records, 48. - Journal of Bryology 38 (3): 235-259. http:// dx.doi.org/10.1080/03736687.2016.1206685

ELLIS, L.T., E. AGCAGIL, M. KÝRMACÝ, M. ALEFFI, V.A. BAKALIN, H. BEDNAREK-OCHYRA, B. CYKOWSKA-MARZENCKA et al. 2016b. New national and regional bryophyte records, 49. - Journal of Bryology 38 (4): 327-347. http://dx.doi.org/10.1080/ 03736687.2016.1225777

ELLIS, L.T., O.M. AFONINA, R.L. ANDRIAMIARISOA, H. BEDNAREK-OCHYRA, B. CYKOWSKA-MARZENCKA, M. STRYJAK-BOGACKA, N.E. BELL et al. 2017. New national and regional bryophyte records, 53. - Journal of Bryology 39 (4): 368-387. http://dx.doi.org/10.1080/03736687.2017.1384204

ELLIS, L.T., O.M. AFONINA, G.YA. DOROSHINA, C. AGUDELO, R.L. ANDRIAMIARISOA, A.K. ASTHANA, D. GUPTA et al. 2019a. New national and regional bryophyte records, 58. - Journal of Bryology 41 (1): 63-84. http://dx.doi.org/10.1080/03736687.2018.1559636

ELLIS, L.T., M. ALEFFI, A. BąCZKIEWICZ, K. BUCZKOWSKA, B. BAMBE, M. BOIKO, N. ZAGORODNIUK et al. 2019b. New national and regional bryophyte records, 60. - Journal of Bryology 41 (3): 285-299. http://dx.doi.org/10.1080/03736687.2019.1643117

ELLIS, L.T., O.M. AFONINA, I.V. CZERNYADJEVA, T.G. IVCHENKO, S.S. HOLOD, V.M. KOTKOVA, E.Y. KUZMINA et al. 2019c. New national and regional bryophyte records, 61. - Journal of Bryology 41 (4): 364-384. http://dx.doi.org/10.1080/ 03736687.2019 .1673601

ELLIS, L.T., C. AH-PENG, G. ASLAN, V.A. BAKALIN, A. BERGAMINI, D.A. CALLAGHAN, P. CAMPISI et al. 2021. New national and regional bryophyte records, 65. - Journal of Bryology 43 (1): 67-91. http://dx.doi.org/10.1080/03736687.2021.1878804
ELVEBAKK, A. 1999. Bioclimatic delimitation and subdivision of the Arctic. - in: Nordal, I. \& Razzhivin, V.Y. (eds.) The species concept in the High North - A Panarctic Flora Initiative. The Norwegian Academy of Science and Letters, Oslo, pp. 81-112.

ENGEL, J.J. 1981. Haplomitrium monoicum, a remarkable new species of Calobryales (Hepaticae) from New Caledonia, together with a reclassification of subg. Haplomitrium. - Annals of the Missouri Botanical Garden 68 (4): $668-676$.

ENGEL, J. J. \& D. GLENNY. 2008. A flora of the liverworts and hornworts of New Zealand, Volume 1. - Missouri Botanical Garden: St. Louis. $897 \mathrm{pp}$.

ENGEL, J. J. \& G. L. MERRILL. 1998. Austral Hepaticae. 25. Krunodiplophyllum Grolle and a revision of the Australasian species of Diplophyllum (Dum.) Dum. (Scapaniaceae, Jungermanniales). - Journal of the Hattori Botanical Laboratory 84: 241-283.

EUROLA, S. 1968. Über die Fjeldheidevegetation in den Gebieten von Isfjorden und Hornsund in Westspitzbergen.-Aquilo. Ser. Botanica 7: $1-56$.

EUROLA, S. 1971. The middle arctic mire vegetation in Spitsbergen. Acta Agralia Fennica 123: 87-107.

EVANS, A.W. 1900. Notes on Hepaticae collected in Alaska. - Proceedings of the Washington Academy of Sciences 2: 287-314.

EVANS, A.W. 1903. Odontoschisma macounii and its North American allies. - Botanical Gazette 36 (5): 321-348.

EVANS, A.W. 1911. Notes on North American Hepaticae. II. - Bryologist 14 (5): 84-88. http://dx.doi.org/10.2307/3237443

FEDOSOV, V.E., E.A. BOROVICHEV, E.A. IGNATOVA\& V.A. BAKALIN. 2015. The bryophyte flora of Eriechka River upper course (SE Taimyr), with comments on the first record of Pseudoditrichum mirabile in Asia. - Arctoa 24 (1): 165-186. http://dx.doi.org/10.15298/ arctoa. 24.17

FELDBERG, K., J. HENTSCHEL, A. BOMBOSCH, D.G. LONG, J. VÁŇA \& J. HEINRICHS. 2009. Transfer of Gottschelia grollei, G. patoniae and Scaphophyllum speciosum to Solenostoma based on chloroplast DNA rbcL sequences. - Plant Systematics and Evolution 280 (3/4): 243-250. http://dx.doi.org/10.1007/s00606-009-0187-3

FELDBERG, K., J. VÁŇA, D.G. LONG, A.J. SHAW, J. HENTSCHEL \& J. HEINRICHS. 2010. A phylogeny of Adelanthaceae (Jungermanniales, Marchantiophyta) based on nuclear and chloroplast DNA markers, with comments on classification, cryptic speciation and biogeography. - Molecular Phylogenetics and Evolution 55 (1): 293-304. http:/ /dx.doi.org/10.1016/j.ympev.2009.11.009

FELDBERG, K., J. HEINRICHS, A.R. SCHMIDT, J. VÁŇA \& H. SCHNEIDER. 2013. Exploring the impact of fossil constraints on the divergence time estimates of derived liverworts. - Plant Systematics and Evolution 299 (3): 585-681. http://dx.doi.org/10.1007/s00606012-0745-y

FELDBERG, K., J. VÁŇA, J. KRUSCHE, J. KRETSCHMANN, S.D.F. PATZAK, O.A. PÉREZ-ESCOBAR, N.R. RUDOLF, N. SEEFELDER, A. SCHÄFER-VERWIMP, D.G. LONG, H. SCHNEIDER \& J. HEINRICHS. 2016. A phylogeny of Cephaloziaceae (Jungermanniopsida) based on nuclear and chloroplast DNA markers. - Organisms, Diversity and Evolution 16 (4): 727-742. http://dx.doi.org/10.1007/s13127016-0284-4

FØRLAND, E.J., R. BENESTAD, I. HANSSEN-BAUER, J.E. HAUGEN \& T. ENGEN SKAUGEN. 2011. Temperature and Precipitation Development at Svalbard 1900-2100. - Advances in Meteorology 2011 (893790): 1-14. http://dx.doi.org/10.1155/2011/893790

FRISVOLL, A.A. 1978. Twenty-eight bryophytes new to Svalbard. - Bryologist 81 (1): 122-136. http://dx.doi.org/10.2307/3242276

FRISVOLL, A.A. 1981. Fifteen bryophytes new to Svalbard, including notes on some rare and interesting species. - Lindbergia 7 (2): 91-102.

FRISVOLL, A.A. \& H.H. BLOM. 1992. Trua moser i Norge med Svalbard; raud liste. - Norsk Institutt for Naturforskning (NINA) Utredning 42: 1-55. 
FRISVOLL, A.A. \& H.H. BLOM. 1997. Trua moser i Noreg med Svalbard. Førebelse ark. - NTNU Vitenskapsmuseet, Botaniske Notat 19973: $1-170$.

FRISVOLL,A.A. \& A. ELVEBAKK. 1996. Acatalogue of Svalbard plants, fungi, algae and cyanobacteria. Part 2. Bryophytes. - Norsk Polarinstitutt Skrifter 198: 57-172.

FRYE, T. C. \& L. CLARK. 1943. Hepaticae of North America, part II. University of Washington Publications in Biology 6: 163-336.

FRYE, T. C. \& L. CLARK. 1945. Hepaticae of North America part III. University of Washington Publications in Biology 6 (3): 337-564.

FRYE, T. C. \& L. CLARK. 1946. Hepaticae of North America, part IV. University of Washington Publications in Biology 6 (4): 565-733.

GEISSLER, P. \& H. BISCHLER. 1985. Index hepaticarum vol. 10. Lembidium to Mytilopsis. - J. Cramer: Berlin. $352 \mathrm{pp}$.

GEISSLER, P. \& H. BISCHLER. 1987. Index hepaticarum vol. 8/9. Jungermannia to Lejeunites. - J. Cramer: Berlin. 310 pp.

GEISSLER, P. \& H. BISCHLER. 1990. Index hepaticarum vol. 12. Racemigemma to Zoopsis. - J. Cramer: Berlin. 337 pp.

GODFREY, J.D. 1977. Hepaticae and Anthocerotae of southwestern British Columbia. $-\mathrm{PhD}$ thesis, University of British Columbia: Vancouver. $433 \mathrm{pp}$.

GODFREY, J.D. \& G.A. GODFREY. 1978. Scapania hians in Shensi, China and British Columbia, Canada. - Bryologist 81 (3): 357-367. http://dx.doi.org/10.2307/3242238

GODFREY, J.D. \& W.B. SCHOFIELD. 1979. New and interesting hepatics from British Columbia, Canada, and northern Washington State, USA, 2. - Bryologist 82 (2): 162-170. http://dx.doi.org/10.2307/3242075

GOTTSCHE, C. M., J. B. G. LINDENBERG \& S. G. NEES VON ESENBECK. 1845. Synopsis hepaticarum, fasc. 2. - Meissner: Hamburg. 304 pp. http://dx.doi.org/10.5962/bhl.title.15221

GOTTSCHE, C. M., J. B. G. LINDENBERG \& S. G. NEES VON ESENBECK. 1846. Synopsis hepaticarum, fasc. 4. - Meissner: Hamburg. 624 pp. http://dx.doi.org/10.5962/bhl.title.15221

GOTTSCHE, C. M., J. B. G. LINDENBERG \& S. G. NEES VON ESENBECK. 1847. Synopsis hepaticarum, fasc. 5. - Meissner: Hamburg. 834 pp. http://dx.doi.org/10.5962/bhl.title.15221

GRADSTEIN, S.R. \& A.L. ILKIU-BORGES. 2015. A taxonomic revision of the genus Odontoschisma (Marchantiophyta: Cephaloziaceae). - Nova Hedwigia 100 (1/2): 15-100. http://dx.doi.org/10.1127/ nova_hedwigia/2014/0219

GRADSTEIN, S. R. \& J. VÁŇA. 1987. On the occurrence of Laurasian liverworts in the Tropics. - Memoirs of the New York Botanical Garden 45: 388-425.

GROLLE, R. 1960. Beitrag zur Kenntnis von Barbilophozia, inbesonderes B. floerkei und B. hatcheri. - Nova Hedwigia 2: 555-566.

GROLLE, R. 1964. Miscellanea hepaticologica 1-10.- Österreichische Botanische Zeitschrift 111 (2/3): 185-192. http://dx.doi.org/10.1007/ BF01373763

GROLLE, R. 1965. Harpanthus drummondii - ein Lebermoosendemit des östlichen Nordamerika. - Österreichische Botanische Zeitschrift 112 (3): 268-284. http://dx.doi.org/10.1007/BF01372951

GROLLE, R. 1967. Miscellanea hepaticologica 71-80.-Transactions of the British Bryological Society 5 (2): 271-282. http://dx.doi.org/ 10.1179/006813867804804179

GROLLE, R. 1976. Verzeichnis der Lebermoose Europas und benachbarter Gebiete. - Feddes Repertorium 87 (3/4): 171-279. http://dx.doi.org/ 10.1002/fedr.19760870303

GROLLE, R. \& D.G. LONG. 2000. An annotated check-list of the Hepaticae and Anthocerotae of Europe and Macaronesia. - Journal of Bryology 22 (2): 103-140. http://dx.doi.org/10.1179/jbr.2000.22.2.103

GUGNACKA-FIEDOR, W. \& B. NORYÚKIWICZ. 1982. The vegetation of Kaffiøyra, Oscar II Land, NW Spitsbergen. - Acta Universitatis Nicolai Copernici. Nauki matematyczno-przyrodnicze, Geographia) 51: 203-238.
HADAČ, E. 1946. The plant-communities of Sassen Quarter, Vestspitsbergen. - Studia Botanica Cechoslovaca 7: 127-164.

HADAČ, E. 1989. Notes on plant communities of Spitsbergen. - Folia Geobotanica et Phytotaxonomica 24 (2): 131-169.

HAGEN, I. 1908. The mosses and hepatics of Prince Charles Foreland, Spitsbergen. - Transactions and Proceedings of the Botanical Society of Edinburgh 23 (1/4): 326-330. http://dx.doi.org/10.1080/ 03746600809469176

HAGEN, A. 1952. Plants collected in Vestspitsbergen in the summer of 1933. - Norsk Polarinstitutt meddelelser 70: 1-14.

HARMSEN, L. \& G. SEIDENFADEN. 1932. Mosses of the Godthaab expedition, 1928. - Meddelelser om Grønland 82 (2): 1-42.

HÄSSEL, G.G. \& M.F. RUBIES. 2009. Catalogue of Marchantiophyta and Anthocerotophyta of southern South America. - Nova Hedwigia Beiheft 134: 1-672.

HEINEMEIJER, H.D. 1979. De vegetatie van het westelijk gedeelte van Rosenbergdalen, Edgeøya, Svalbard. Results Reindeer Environment Expedition Svalbard. - Univ. Amsterdam, Research groep Oecologie van Cormophyta en Vegetatiekunde, Intern Rapp. 67: 1-63.

HEINEMIJER, H.D. \& A.J. VAN DIJK. 2004. Rosenbergdalen, green valley in the barrenland of Edgeøya, Spitsbergen - in: Boschman, L. \& L. Haquebord, Eds. Permanence in diversity. Netherlands ecological research on Edgeøya Spitsbergen. Circumpolar Studies 1. Groningen: Barkhuis Publishing, pp. 46-69.

HEINRICHS, J., J. HENTSCHEL, R. WILSON, K. FELDBERG \& H. SCHNEIDER. 2007. Evolution of leafy liverworts (Jungermanniidae, Marchantiophyta): estimating divergence times from chloroplast DNA sequences using penalized likelihood with integrated fossil evidence. Taxon 56 (1): 31-44. http://dx.doi.org/10.2307/25065733

HEINRICHS, J., A. BOMBOSCH, K. FELDBERG, H.-P. KREIER, J. HENTSCHEL, J. ECKSTEIN, D.G. LONG, R.-L. ZHU, A. SCHÄFER-VERWIMP, A.R. SCHMIDT, B. SHAW, A.J. SHAW \& J. VÁŇA. 2012. A phylogeny of the northern temperate leafy liverwort genus Scapania (Scapaniaceae, Jungermanniales). - Molecular Phylogenetics and Evolution 62 (3): 973-985. http://dx.doi.org/10.1016/ j.ympev.2011.11.029

HENTSCHEL, J., R. WILSON, M. BURGHARDT, H.-J. ZÜNDORF, H. SCHNEIDER \& J. HEINRICHS. 2006. Reinstatement of Lophocoleaceae (Jungermanniopsida) based on chloroplast gene rbcL data: exploring the importance of female involucres for the systematics of Jungermanniales. - Plant Systematics and Evolution 258 (2): 211226. http://dx.doi.org/10.1007/s00606-006-0408-y

HENTSCHEL, J., J.A. PATON, H. SCHNEIDER \& J. HEINRICHS. 2007. Acceptance of Liochlaena Nees and Solenostoma Mitt., the systematic position of Eremonotus Pearson and notes on Jungermannia L. s. 1. (Jungermanniidae) based on chloroplast DNA sequence data. Plant Systematics and Evolution 268 (1/4): 147-157. http://dx.doi.org/ 10.1007/s00606-007-0549-7

HERZOG, T. 1926. Die Geographie der Moose. - Gustav Fischer: Jena. $439 \mathrm{pp}$.

HODGETTS, N.G. 2015. Checklist and country status of European bryophytes - towards a new Red List for Europe. - Irish Wildlife Manual 84: $1-125$.

HODGETTS, N.G. \& N. LOCKHART. 2020. Checklist and country status of European bryophytes - update 2020. - Irish Wildlife Manual 123: $1-214$.

HODGETTS, N.G., L. SÖDERSTRÖM, T.L. BLOCKEEL, S. CASPARI, M.S. IGNATOV, N.A. KONSTANTINOVA, N. LOCKHART, B. PAPP, C. SCHRÖCK, M. SIM-SIM, D. BELL, N.E. BELL, H.H. BLOM, M.A. BRUGGEMAN-NANNENGA, M. BRUGUÉS, J. ENROTH, K.I. FLATBERG, R. GARILLETI, L. HEDENÄS, D.T. HOLYOAK, V. HUGONNOT, I. KARIYAWASAM, H. KÖCKINGER, J. KUČERA, F. LARA \& R.D. PORLEY. 2020. An annotated checklist of bryophytes of Europe, Macaronesia and Cyprus. - Journal of Bryology 42 (1): 1116. http://dx.doi.org/10.1080/03736687.2019.1694329 
HOFFMAN, W. 1968. Geobotanische Untersuchungen in Südost-Spitzbergen 1960. - Franz Steiner Verlag: Wiesbaden. 83 pp.

HOOKER, W.J. 1828. Botanical appendix - in: Parry, W.E., Ed. Narrative of an attempt to reach the North Pole in boats fitted for the purpose, and attached to His Majesty's ship Hecla, in the year MDCCCXXVII, under the command of captain William Edward Parry. London: John Murray, pp. 207-220.

HORIKAWA, Y. 1950. Symbolae florae Bryophytae Orientali-Asiae et Micronesiae XI. - Hikobia 1: 30-36.

INOUE, H. 1988. Bryophyta Selecta Exsiccata Fasc. XIX (No. 901 - 950). - Natural Science and Museum: Tokyo. 6 pp.

JØRGENSEN, E. 1934. Norges levermossor.-Bergens Museums Skrifter (n.ser.) 16: 1-343.

KAALAAS, B. 1893a. Levermosernes udbredelse i Norge. - Nyt Magazin for Naturvidenskaberne 33 (1/2): 1-192.

KAALAAS, B. 1893b. Levermosernes udbredelse i Norge. - Nyt Magazin for Naturvidenskaberne 33 (3): 193-288.

KAALAAS, B. 1893c. Levermosernes udbredelse i Norge. - Nyt Magazin for Naturvidenskaberne 33 (4/5): 289-490.

KALINAUSKAITË, N. \& M. VON CRÄUTLEIN. 2010. Lophozia murmanica Kaal. - a new species found in Finland. - Bryobrotherella 13: 119-121.

KARCZMARZ, K. \& F. ŚWIĘS. 1988. Bryophyte flora of south Bellsund Bay coast (western Spitsbergen) - in: Repelewska-Pękalowa, J., K. Pękala \& M. Harasimiuk, Eds. Polar Session "Natural environment research Bellsund, Spitsbergen”. Lublin: Maria Curie-Sklodowska Univ., pp. 229-235.

KARCZMARZ, K. \& F. ŚWIĘS. 1989. Mosses (Bryophyta) of regions of Lognedalsflya, Dyrstadflya and northern part of Chamberlindalen on south-east shore of Bellsund (West Spitsbergen - in: RepelewskaPękalowa, J. \& K. Pękala, Eds. Polar session. Arctic environment research. Lublin: Maria Curie-Sklodowska Univ., pp. 89-96.

KARCZMARZ, K. \& F. ŚWIĘS. 1990a. Bryophytes collected in arctic tundra of the eastern slopes of Activekammen (Western Spitsbergen) in 1987-1988 - in: Repelewska-Pękalowa, J. \& K. Pękala, Eds. Polar session. "Periglacial phenomena of Western Spitsbergen". Lublin, Poland., pp. 175-183.

KARCZMARZ, K. \& F. ŚWIĘS. 1990b. Bryophyte collected in Arctic Tundra of Dyrstad Region (Western Spitsbergen) in 1988. - Annales Universitatis Mariae Curie-Skłodowska, C Biologia 45 (12): 127 139.

KARLSSON, T. 1993. Mossor. - Svensk Botanisk Tidskrift 87: 104-106.

KLIMOVA, K.G. \& V.A. BAKALIN. 2017. Two Scapania species (Scapaniaceae) newly recorded from Kamchatka. - Arctoa 26 (2): 125-131. http://dx.doi.org/10.15298/arctoa.26.12

KOBAYASHI, K., H. KASHIWADANI \& H. DEGUCHI. 1990. Vegetation of Bohemanflya in Spitsbergen - in: Tatsumi, T., Ed. The Japanese scientific expeditions to Svalbard 1983-1988. Tokyo, pp. 2970.

KONSTANTINOVA, N.A. 2000. Distribution patterns of the north holarctic hepatics. - Arctoa 9: 29-94. http://dx.doi.org/10.15298/arctoa.09.06

KONSTANTINOVA, N.A. 2003. On phytogeography of hepatics Spitzbergen archipelago - in: Matishov, G.G. \& G.A. Tarasov, Eds. Complex investigations of Spitzbergen nature, issue 3. Apatiti, pp. 163-165.

KONSTANTINOVA, N.A. \& N.E. KOROLEVA. 2003. On some dwarf forms of hepatics new for Spitzbergen archipelago-in: Matishov, G.G. $\&$ G.A. Tarasov, Eds. Complex investigations of Spitzbergen nature, issue 3. Apatiti, pp. 156-161.

KONSTANTINOVA, N.A. \& A.N. SAVCHENKO. 2006. Findings of some rare hepatics in Spitsbergen in western coast of Bockfjorden - in: Matishov, G.G. \& G.A. Tarasov, Eds. Complex investigations of Spitzbergen nature, issue 6. Apatiti, pp. 330-336.

KONSTANTINOVA, N.A. \& A.N. SAVCHENKO. 2007. New for archi- pelago and rare in Spitsbergen hepatics in vicinities of Barentsburg in: Matishov, G.G. \& G.A. Tarasov, Eds. Complex investigations of Spitzbergen nature, issue 7. Apatiti, pp. 265-278.

KONSTANTINOVA, N.A. \&A.N. SAVCHENKO. 2008a. Contributions to the hepatic flora of Svalbard. - Lindbergia 33 (1): 13-22.

KONSTANTINOVA, N.A. \& A.N. SAVCHENKO. 2008b. Hepatics in: Koroleva, N.E., N.A. Konstantinova, O.A. Belkina, D.A. Davydov, A.J. Lihachev, A.N. Savchenko \& I.N. Urbanavichene., Eds. Flora and vegetation of Grønfjord area (Spitsbergen archipelago). Apatity, pp. 54-80.

KONSTANTINOVA, N.A. \& A.N. SAVCHENKO. 2008c. Distribution of liverworts, rare for the Svalbard Archipelago, around Piramida (Pyramiden) Settlement. - in: Matishov, G.G. \& G.A. Tarasov, Eds. Nature of the shelf and archipelagos of the European Arctic. Iss. 8. Proceedings of the International Scientific Conference (Murmansk, November 9-11, 2008). Geos, Moscow, pp. 177-181.

KONSTANTINOVA, N.A. \& A.N. SAVCHENKO. 2011. On distribution of Scapania ligulifolia R.M.Schust. (Hepaticae) on Svalbard-in: Matishov, G.G., Ed. Global climate processes and their impact on the ecosystems of the Arctic and subarctic regions 11. Apatity, pp. 96-97.

KONSTANTINOVA, N.A. \& A.N. SAVCHENKO. 2012. Contribution to the hepatic flora of the Nordaustlandet (Svalbard). I. Hepatics of the north coast of Murchisonfjorden. - Polish Botanical Journal 57 (1): $181-195$.

KONSTANTINOVA, N.A. \& A.N. SAVCHENKO. 2015. Preliminary results of study Hepatic diversity of the Nordaustlandet (Svalbard). in: History of development of Spitsbergen and northern Eurasia,pp. 19-20.

KONSTANTINOVA, N.A. \& A.N. SAVCHENKO. 2018. Contribution to the hepatic flora of the Svalbard: Hepatics of the Barents Island. Folia Cryptogamica Estonica 55: 105-115. http://dx.doi.org/ 10.12697/fce.2018.55.11

KONSTANTINOVA, N.A. \& A.N. SAVCHENKO. 2020a. Contribution to the hepatic flora of Svalbard Ill: Hepatics of Prins Karls Forland (Forlandet nasjonalpark). - Nova Hedwigia Beiheft 150: 179-192. http://dx.doi.org/10.1127 /nova-suppl/2020/179

KONSTANTINOVA, N.A. \&A.N. SAVCHENKO. 2020b. On the spread of liverworts included in the Red Book of Europe on Svalbard - in: Makarevich, P.R., D.V. Moiseev, A.S. Bulavina \& N.N. Panteleeva, Eds. Complex investigations of Spitsbergen and offshore nature: Proceedings of the XV scientific conference (Murmansk). Apatity: FRC Kola Science Centre RAS, pp. 50-52.

KONSTANTINOVA, N.A. \& A.A. VILNET. 2016. A new species of the genus Jungermannia (Jungermanniales, Marchantiophyta) from the Caucasus with notes on taxa delimitation and taxonomy of Jungermannia s. str. - Phytotaxa 255 (3): 227-239. http://dx.doi.org/ 10.11646/phytotaxa.255.3.4

KONSTANTINOVA, N.A., N.E. KOROLEVA \& A.N. SAVCHENKO. 2009. Study of the vegetation of Spitzbergen - In: Kalinnikov, V.T. Ed. Nauka na Shpitsbergene. Istoriya rossijskikh issledovanij, St. Petersburg, Gamas, pp. 190-205.

KONSTANTINOVA, N.A., A.A. VILNET \& S. ŞTEFĂNUȚ. 2014. On the distribution and variability of Bucegia romanica Radian. - Arctoa 23: 137-144. http://dx.doi.org/10.15298/arctoa.23.11

KONSTANTINOVA, N.A., A.A. VILNET \& YU.S. MAMONTOV. 2020a. A new species of the genus Lophozia (Lophoziaceae) from Svalbard Archipelago. - Arctoa 29 (2): 124-134. http://dx.doi.org/ 10.15298/arctoa.29.10

KONSTANTINOVA, N.A., A.N. SAVCHENKO \& A.A. VILNET. 2020 b. Hepaticae svalbardensia exsiccatae, fasciculus I. (nos. 1-25). - Arctoa 29 (1): 101-104. http://dx.doi.org/10.15298/arctoa.29.08

KONSTANTINOVA, N. A., A. N. SAVCHENKO \& A. A. VILNET. 2020c. Contribution to the liverwort flora of Franz Josef Land Archipelago I. Liverworts (Marchantiophyta) of Ziegler Island. - Arctoa 29 (1): 63-71. doi: http://dx.doi.org/10.15298/arctoa.29.04 
KOROLEVA, N.E. \& E.A BOROVICHEV. 2014. Rare plant communities with abundance of Sauteria alpina (Nees) Nees in the arctic tundra of Svalbard. - Arctoa 23: 5-10. http://dx.doi.org/10.15298/arctoa.23.02

KOSOVICH-ANDERSON, Y.I. 2014. An annotated checklist of bryophytes of the east slope of the Wind River Range, Wyoming / preliminary results of bryophyte inventory. - U.S.D.A. Forest Service General Technical Report PNW. 129 pp.

KREIER, H.-P., K. FELDBERG, F. MAHR, A. BOMBOSCH, A.R. SCHMIDT, R.-L. ZHU, M. VON KONRAT, B. SHAW, A.J. SHAW \& J. HEINRICHS. 2010. Phylogeny of the leafy liverwort Ptilidium: Cryptic speciation and shared haplotypes between the Northern and Southern. - Molecular Phylogenetics and Evolution 57 (3): 1260-1267. http://dx.doi.org/10.1016/j.ympev.2010.10.002

KUC, M. 1998. Environs of the Polish Polar Station (Isbjörnhamna, north Hornsund, Spitsbergen) as surveyed in 1958 by vegetation mapping and related methods - in: Glowacki, P. \& J. Bednarek, Eds. Polish polar studies, 25th International Polar Symposium. Warsaw: Polish Academy of Sciences, pp. 141-157.

KUSNETSOVA, O.I., N.A. KONSTANTINOVA, A.A. VILNET, E.D LAPSHINA, O.M. AFONINA, A.I. MAKSIMOV, T.A. MAKSIMOVA\& M.S. IGNATOV. 2020. Bryophyte molecular barcoding records. 3. - Arctoa 29 (2): 216-218. http://dx.doi.org/10.15298/arctoa.29.15

LADYZHENSKAJA, K.I. \& A.L. ZHUKOVA. 1972. Orthocaulis elongatus (Lindb.) Evans - species rara primum in flora hepaticarum URSS inventa. - Novosti sistematiki nizshikh rastenii 9: 304-307.

LAMMES, T. 1977. Additions to the hepatic flora of Finland. - Annales Botanici Fennici 14 (1): 17-20.

LE GALLO, P.C. 1951. Florule bryologique des Îles Saint-Pierre et Miquelon. - Revue Bryologique et Lichénologique 20 (1/2): 43-93.

LINDBERG, S.O. 1866. Förteckning öfver mossor, insamlade under de svenska expeditionerna till Spitsbergen 1858 och 1861. - Öfversigt af Kongliga Vetenskaps-Akademiens Förhandlingar 23 (10): 535-561.

LINDBERG, S.O. 1877. Utredning af de under namn af Sauteria alpina sammanblandade former. - Botaniska Notiser 30: 73-78.

LINDBERG, S.O. 1882. Monographia praecursoria Peltolepidi, Sauteriae et Cleveae. - Acta Societatis pro Fauna et Flora Fennica 2 (3): $1-15$.

LINDBERG, S.O. \& H.W. ARNELL. 1889. Musci asiae borealis. Beschreibung der von den Schwedischen Expeditionen nach Sibirien in den Jahren 1875 und 1876 gesammelten Moose mit Berücksichtigung aller früheren bryologischen Angaben für das russische Nord-Asien. Erster Theil: Lebermoose. - Kongliga Svenska Vetenskaps-Akademiens Handlingar (n. ser.) 23 (5): 1-69.

LIVESAY, W. 1870. Notice of plants collected in Spitzbergen and Nova Zembla in the summer of 1869. - Transactions of the Botanical Society of Edinburgh 10: 333-341.

LONG, D.G. \& B.J. CRANDALL-STOTLER. 2020. Marchantia platycarpa (Marchantiopsida, Marchantiaceae), a new species from China. - Nova Hedwigia Beiheft 150: 109-116.

LONG, D.G., J.A. PATON \& G.P. ROTHERO. 1990. Marsupella arctica (Berggr.) Bryhn \& Kaal. in Scotland, new to the British Isles. - Journal of Bryology 16 (2): 163-171. http://dx.doi.org/10.1179/ jbr.1990.16.2.163

LONG, D.G., G.P ROTHERO \& J.A. PATON. 2003. Athalamia hyalina (Sommerf.) S.Hatt. in Scotland, new to the British Isles. - Journal of Bryology 25 (4): 253-257. http://dx.doi.org/10.1179/ 037366803225013119

LORENZ, A. 1915. List of Hepaticae to be expected in New England. Bryologist 18 (2): 24-26. http://dx.doi.org/10.2307/3237857

MAMONTOV, Y.S. \& A.A. VILNET. 2013. Anastrophyllum ellipticum Inoue (Jungermanniales, Marchantiophyta), a new species for Russian liverwort flora. - Arctoa 22: 151-158. http://dx.doi.org/10.15298/arctoa. 22.24

MAMONTOV, Y.S. \& A.A. VILNET. 2017. Cephaloziella konstantinovae (Cephaloziellaceae, Marchantiophyta), a new liverwort species from
Russia and Mongolia identifies by integrative taxonomy. - Polish Botanical Journal 62 (1): 1-19. http://dx.doi.org/10.1515/pbj-2017-0001

MAMONTOV, Y.S., N.A. KONSTANTINOVA, A.A. VILNET, A.D. POTEMKIN, E.V. SOFRONOVA \& N.S. GAMOVA. 2018. On resurrection of Marsupella parvitexta Steph. (Gymnomitriaceae, Marchantiophyta) as a semi cryptic species of the genus Gymnomitrion.-Nova Hedwigia 106 (1/2): 81-101. http://dx.doi.org/10.1127/ nova_hedwigia/2017/0466

MAMONTOV, Y.S., A.A. VILNET, N.A. KONSTANTINOVA \& V.A. BAKALIN. 2019. Two more species of Gymnomitriaceae (Marchantiophyta) in the North Pacific. - Botanica Pacifica 8 (1): 67-80. http:/ /dx.doi.org/10.17581/bp.2019.08113

MILLER, H. A., H. O. WHITTIER \& B. A. WHITTIER. 1983. Prodromus florae hepaticarum Polynesiae. Catalogue of Hepaticae and Anthocerotae. - Bryophytorum Bibliotheca 25: 1-423.

MINAMI, Y., S. OKITSU \& H. KANDA. 1997b. Relationship between plant community and topographic factor on the moraine at deglaciated Arctic Terrain in Ny-Ålesund, Svalbard. - Bulletin of faculty of agriculture, Tamagawa University 37: 21-30.

MOHR, C. 1901. Plant life of Alabama. - Contributions from the United States National Herbarium 6: 1-921.

MÜLLER, K. 1900. Vorläufige Bemerkungen zu einer Monographie der Scapania-Arten. - Botanisches Centralblatt 82 (13): 401-411.

MÜLLER, K. 1901. Vorarbeiten zu einer Monographie der Gattung Scapania. - Bulletin de l'Herbier Boissier (sér. 2) 1 (6): 593-614.

MÜLLER, K. 1905. Monographie der Lebermoosgattung Scapania Dum. - Nova Acta Academiae Caesareae Leopoldino-Carolinae Germanicae Naturae Curiosorum 83: 1-312.

MÜLLER, K. 1907a. Die Lebermoose (Dr. L. Rabenhorst's Kryptogamen-Flora von Deutschland, Oesterreich und der Schweiz, 2 Aufl., 6 Band), 1 Abth., 4. - Eduard Kummer: Leipzig.

MÜLLER, K. 1907b. Die Lebermoose (Dr. L. Rabenhorst's Kryptogamen-Flora von Deutschland, Oesterreich und der Schweiz, 2 Aufl., 6 Band), 1 Abth., 5. - Eduard Kummer: Leipzig.

MÜLLER, K. 1909a. Die Lebermoose (Dr. L. Rabenhorst's Kryptogamen-Flora von Deutschland, Oesterreich und der Schweiz, 2 Aufl., 6 Band), 1 Abth., 7. - Eduard Kummer: Leipzig.

MÜLLER, K. 1909b. Die Lebermoose (Dr. L. Rabenhorst's Kryptogamen-Flora von Deutschland, Oesterreich und der Schweiz, 2 Aufl., 6 Band), 1 Abth., 8. - Eduard Kummer: Leipzig.

MÜLLER, K. 1909c. Die Lebermoose (Dr. L. Rabenhorst's Kryptogamen-Flora von Deutschland, Oesterreich und der Schweiz, 2 Aufl., 6 Band), 1 Abth., 9. - Eduard Kummer: Leipzig.

MÜLLER, K. 1910a. Die Lebermoose (Dr. L. Rabenhorst's Kryptogamen-Flora von Deutschland, Oesterreich und der Schweiz, 2 Aufl., 6 Band), 1 Abth., 10. - Eduard Kummer: Leipzig.

MÜLLER, K. 1910b. Die Lebermoose (Dr. L. Rabenhorst's Kryptogamen-Flora von Deutschland, Oesterreich und der Schweiz, 2 Aufl., 6 Band), 1 Abth., 11. - Eduard Kummer: Leipzig.

MÜLLER, K. 1911. Die Lebermoose (Dr. L. Rabenhorst's KryptogamenFlora von Deutschland, Oesterreich und der Schweiz, 2 Aufl., 6 Band), 1 Abth., 14. - Eduard Kummer: Leipzig.

MÜLLER, K. 1913a. Die Lebermoose (Dr. L. Rabenhorst's Kryptogamen-Flora von Deutschland, Oesterreich und der Schweiz, 2 Aufl., 6 Band), 2 Abth., 17. - Eduard Kummer: Leipzig.

MÜLLER, K. 1913b. Die Lebermoose (Dr. L. Rabenhorst's Kryptogamen-Flora von Deutschland, Oesterreich und der Schweiz, 2 Aufl., 6 Band), 2 Abth., 18. - Eduard Kummer: Leipzig.

MÜLLER, K. 1914a. Die Lebermoose (Dr. L. Rabenhorst's Kryptogamen-Flora von Deutschland, Oesterreich und der Schweiz, 2 Aufl., 6 Band), 2 Abth., 19. - Eduard Kummer: Leipzig.

MÜLLER, K. 1914b. Die Lebermoose (Dr. L. Rabenhorst's Kryptogamen-Flora von Deutschland, Oesterreich und der Schweiz, 2 Aufl., 6 Band), 2 Abth., 20. - Eduard Kummer: Leipzig. 
MÜLLER, K. 1915a. Die Lebermoose (Dr. L. Rabenhorst's Kryptogamen-Flora von Deutschland, Oesterreich und der Schweiz, 2 Aufl., 6 Band), 2 Abth., 21. - Eduard Kummer: Leipzig.

MÜLLER, K. 1915b. Die Lebermoose (Dr. L. Rabenhorst's Kryptogamen-Flora von Deutschland, Oesterreich und der Schweiz, 2 Aufl., 6 Band), 2 Abth., 22. - Eduard Kummer: Leipzig.

MÜLLER, K. 1952. Die Lebermoose Europas (Dr. L. Rabenhorst's Kryptogamen-Flora von Deutschland, Österreich und der Schweiz, 3 Aufl., 6 Band), 1 Abth., 3 Lieferung. - Akademische Verlagsgesellschaft: Leipzig.

MÜLLER, K. 1954a. Die Lebermoose Europas (Dr. L. Rabenhorst's Kryptogamen-Flora von Deutschland, Österreich und der Schweiz, 3 Aufl., 6 Band), 1 Abth., 4 Lieferung. - Akademische Verlagsgesellschaft: Leipzig.

MÜLLER, K. 1954b. Die Lebermoose Europas (Dr. L. Rabenhorst's Kryptogamen-Flora von Deutschland, Österreich und der Schweiz, 3 Aufl., 6 Band), 1 Abth., 5 Lieferung. - Akademische Verlagsgesellschaft: Leipzig.

MÜLLER, K. 1956a. Die Lebermoose Europas (Dr. L. Rabenhorst's Kryptogamen-Flora von Deutschland, Österreich und der Schweiz, 3 Aufl., 6 Band), 2 Abth., 6 Lieferung. - Akademische Verlagsgesellschaft: Leipzig.

MÜLLER, K. 1956b. Die Lebermoose Europas (Dr. L. Rabenhorst's Kryptogamen-Flora von Deutschland, Österreich und der Schweiz, 3 Aufl., 6 Band), 2 Abth., 7 Lieferung. - Akademische Verlagsgesellschaft: Leipzig.

MÜLLER, K. 1956c. Die Lebermoose Europas (Dr. L. Rabenhorst's Kryptogamen-Flora von Deutschland, Österreich und der Schweiz, 3 Aufl., 6 Band), 2 Abth., 8 Lieferung. - Akademische Verlagsgesellschaft: Leipzig.

NEWSHAM, K.K. \& W.P. GOODALL-COPESTAKE. 2021. Liverworts frequently form mycothalli on Spitsbergen in the High Arctic - Polar Research, n.s. 40 (7727): 1-13.

NILSEN, L. \& A. ELVEBAKK. 2014. Vegetation of exposed calcareous ridges in central Spitsbergen, Svalbard, Norway. - Phytocoenologia 44 (1/2): 19-29. http://dx.doi.org/10.1127/0340-269X/2013/00430470

NORWEGIAN POLAR INSTITUTE. 2003. The place names of Svalbard. - Norsk polarinstitutt Rapportserie 122: 1-537.

NUTH, C., J. KOHLER, M. KÖNIG, A. VON DESCHWANDEN, J.O. HAGEN, A. KÄÄB, G. MOHOLDT \& R. PETTERSSON. 2013. Decadal changes from a multi-temporal glacier inventory of Svalbard. The Cryosphere 7: 1603-1621. DOI: https://doi.org/10.5194/tc-71603-2013

OCHYRA, R. \& J. VÁŇA. 1989. The hepatics of King George Island, South Shetland Islands, Antarctica, with particular reference to the Admirality Bay region. - Polish Polar Research 10 (2): 183-210.

OLSON, D.M., E. DINERSTEIN, E.D. WIKRAMANAYAKE, N.D. BURGESS, G.V.N. POWELL, E.C. UNDERWOOD, J.A. D'AMICO, I. ITOUA, H.E. STRAND, J.C. MORRISON, C.J. LOUCKS, T.F. ALLNUTT, T.H. RICKETTS, Y. KURA, J.F. LAMOREUX, W.W. WETTENGEL, P. HEDAO \& K.R. KASSEM. 2001. Terrestrial ecoregions of the world: a new map of life on earth - BioScience 51 (11): 933-938.

PARIHAR, N.S., B. LAL \& N. KATIYAR. 1994. Hepatics and Anthocerotes of India. A new annotated checklist. - Indian Universities Press: Allahabad.

PATON, J. A. 1999. The liverwort flora of the British Isles. - Harley Books: Colchester. $626 \mathrm{pp}$.

PATZAK, S.D.F., J. VÁŇA, M.A.M. RENNER \& J. HEINRICHS. $2016 \mathrm{a}$. Transfer of the leafy liverwort Xenochila from Plagiochilaceae (Lophocoleineae) to Jungermanniaceae. - Plant Systematics and Evolution 302 (7): 891-899. http://dx.doi.org/10.1007/s00606-016-1305-7

PATZAK, S.D.F., A. SCHÄFER-VERWIMP, J. VÁŇA, M.A.M. RENNER, D.F. PERALTA\& J. HEINRICHS. 2016b. Chonecoleaceae (Lo- phocoleineae) is a synonym of Cephaloziellaceae (Cephaloziineae) and Rivulariella (Jungermanniineae) belongs to Scapaniaceae s.l. (Cephaloziineae). - Phytotaxa 267 (2): 91-102. http://dx.doi.org/10.11646/ phytotaxa.267.2.1

PERSSON, H. 1942. Arctic bryophytes mainly collected by rev. J. Lagerkranz. - Svensk Botanisk Tidskrift 36: 444-450.

PERSSON, H. 1946. Some Alaskan and Yukon bryophytes. - Bryologist 49 (2): 41-58. http://dx.doi.org/10.2307/3239692

PERSSON, H. 1952. Critical or otherwise interesting bryophytes from Alaska-Yukon. - Bryologist 55 (1): 1-25. http://dx.doi.org/10.2307/ 3240270

PERSSON, H. 1962. Bryophytes from Alaska collected by E. Hultén and others. - Svensk Botanisk Tidskrift 56: 1-35.

PERSSON, H. \& O. GJAEREVOLL. 1961. New records of Alaskan bryophytes. - Det Kongelige Norske Videnskabers Selskabs Forhandlinger 1961-2: $1-26$.

PERSSON, H. \& L.A. VIERECK. 1983. Collections and discussions of some bryophytes from Alaska. - Lindbergia 9 (1): 5-20.

PHILIPPI, G. 1973. Moosflora und Moosvegetation des Freeman-SundGebietes (Südost-Spitzbergen). - Franz Steiner Verlag: Wiesbaden. 83 pp.

PHIPPS, C.J. 1774. A voyage towards the north pole undertaken by his Majesty's command. - J. Nourse: London. $253 \mathrm{pp}$.

PHIPS, C.I. 1777. Reise nach dem Nordpol. Auf Befehl Ihro Königl. Grossbrittannischen Majestät. Unternommen im Jahr 1773. - Bern. 304 pp. http://dx.doi.org/10.3931/e-rara-28783

POLUNIN, N. 1945. Plant life in Kongsfjord, West Spitsbergen. - Journal of Ecology 33 (1): 82-108. http://dx.doi.org/10.2307/2256560

POTEMKIN, A.D. 1994. Studies on Scapania, Hepaticae sectio Rufidulae and section Nemorosae. - Journal of the Hattori Botanical Laboratory 77: 273-285.

POTEMKIN, A.D. 1999. An analysis of the practical taxonomy of some critical northern species of Scapania (Scapaniaceae, Hepaticae). - Bryologist 102 (1): 32-38. http://dx.doi.org/10.2307/3244456

POTEMKIN, A.D. 2014. Contribution to the liverwort flora of the Russian Arctic: Champ, Heiss, Vize, Troynoy and Vaygach islands. - Novosti sistematiki nizshikh rastenii 48: 374-379.

PRESTØ, T., M. LÜTH \& K. HASSEL. 2014. Bryophytes of the Longyearbyen area. - NTNU Vitenskapsmuseet Naturhistorisk Notat 199410: $1-68$.

PREY, T., BOUDIER, P. \& J. WERNER. 2014. Cephaloziella uncinata (Cephaloziellaceae, Marchantiophyta) en Haute-Normandie, une hépatique arctique nouvelle. - Cryptogamie, Bryologie 35 (3): 313-320. http://dx.doi.org/10.7872/cryb.v35.iss3.2014.313

REJMENT-GROCHOWSKA, I. 1967. Contribution to the hepatic flora of the north coast of Hornsund (S. W. Svalbard). - Acta Societatis Botanicorum Poloniae 36 (3): 531-544.

RØNNING, O.I. 1996. The flora of Svalbard. Polarhåndbok No. 10.Norsk polarinstitutt. $184 \mathrm{pp}$.

RUBASINGHE, S.C.K. 2011. Phylogeny and taxonomy of the complex thalloid liverwort family Cleveaceae Cavers. - Royal Botanic Garden: Edinburgh. 264 pp.

SANIEWSKI, M., P. WIETRZYK-PEŁKA, T. ZALEWSKA, M.A. OLECH, M. HUBERT WĘGRZYN. 2020. Bryophytes and lichens as fallout originated radionuclide indicators in the Svalbard Archipelago (high Arctic). - Polar Science 25 (100536): 1-6. http://dx.doi.org/10.1016/ j.polar.2020.100536

SCHÄFER-VERWIMP, A. \& C. GIANCOTTI. 1993. New or interesting records of Brazilian bryophytes, IV. - Hikobia 11: 285-293.

SCHÄFER-VERWIMP, A., P. MAIR \& I. VERWIMP. 2019. Neue und bemerkenswerte Moosfunde für Südtirol (Provinz Bozen, Italien). Gredleriana, Veröffentlichungen des Naturmuseums Südtirol 19: 514. http://dx.doi.org/10.5281/zenodo.3565279 
SCHIFFNER, V. 1893. Hepaticae - in: Engler, A. \& K. Prantl, Eds. Die Natürlichen Pflanzenfamilien, Teil. I, Abt. 3. Leipzig: Engelmann, pp. 1-141. http://dx.doi.org/10.5962/bhl.title.4635

SCHIFFNER, V. 1898. Conspectus hepaticarum archipelagi indici. Staatsdruckerei: Batavia. $382 \mathrm{pp}$.

SCHIFFNER, V. 1912. Kritik der Europäischen Formen der Gattung Chiloscyphus auf phylogenetischer Grundlage. - Beihefte zum Botanischen Centralblatt. Abt. 2, Systematik, Pflanzengeographie, angewandte Botanik 29: 74-116.

SCHILL, D.B. \& D.G. LONG. 2003. A revision of Anastrophyllum (Spruce) Steph. (Jungermanniales, Lophoziaceae) in the Himalayan region and western China. - Journal of the Hattori Botanical Laboratory 94: 115-157.

SCHLJAKOV, R.N. 1976. Pečenočnye Mchi Severa SSSR. 1. - Nauka: Leningrad. $91 \mathrm{pp}$.

SCHLJAKOV, R.N. 1979. Pečenočnye Mchi Severa SSSR. 2. - Nauka: Leningrad. $191 \mathrm{pp}$.

SCHLJAKOV, R.N. 1980. Pečenočnye Mchi Severa SSSR. 3. - Nauka: Leningrad. $188 \mathrm{pp}$.

SCHLJAKOV, R.N. 1981. Pečenočnye Mchi Severa SSSR. 4. - Nauka: Leningrad. $221 \mathrm{pp}$.

SCHOFIELD, W.B. \& H. CRUM. 1972. Disjunctions in bryophytes. Annals of the Missouri Botanical Garden 59: 174-202.

SCHUMACKER, R., Z. SOLDÁN \& L. MISERERE. 1999. Haplomitrium hookeri (Sm.) Nees (Calobryales, Marchantiophyta), new for Italy in Valsoera (Gran Paradiso National Park,. - Bollettino del Museo Regionale di Scienze Naturali, Torino 16: 89-96.

SCHUSTER, R.M. 1950. Notes on nearctic Hepaticae. II. The Hepaticae in east coast of Hudson Bay. - Bulletin of the National Museum of Canada 122: 1-62.

SCHUSTER, R.M. 1958. Notes on nearctic Hepaticae. XIII: Tritomaria (Lophoziaceae) in Arctic. - Canadian Journal of Botany 36 (2): 269 288. http://dx.doi.org/10.1139/b58-023

SCHUSTER, R.M. 1961. Notes on nearctic Hepaticae. XVIII. New Lophoziaceae from the arctic archipelago of Canada. - Canadian Journal of Botany 39 (4): 965-992. http://dx.doi.org/10.1139/b61-081

SCHUSTER, R.M. 1966. The Hepaticae and Anthocerotae of North America. I. - Columbia University Press: New York. 802 pp.

SCHUSTER, R.M. 1967. Studies on Hepaticae. XV. Calobryales. - Nova Hedwigia 13 (1/2): 1-76.

SCHUSTER, R.M. 1969. The Hepaticae and Anthocerotae of North America. II. - Columbia University Press: New York. 1062 pp.

SCHUSTER, R.M. 1974. The Hepaticae and Anthocerotae of North America. III. - Columbia University Press: New York. 880 pp.

SCHUSTER, R.M. 1980. The Hepaticae and Anthocerotae of North America. IV. - Columbia University Press: New York. 1334 pp.

SCHUSTER, R.M. 1981. Late Pleistocene bryological relicts in Western Massachusetts. - Rhodora 83: 441-448.

SCHUSTER, R.M. 1983. Phytogeography of the Bryophyta - in: Schuster, R.M., Ed. New Manual of Bryology, vol. 1. Nichinan: Hattori Botanical Laboratory, pp. 463-626.

SCHUSTER, R.M. 1992. The Hepaticae and Anthocerotae of North America. VI. - Columbia University Press: New York. 937 pp.

SCHUSTER, R.M. \& K. DAMSHOLT. 1974. The Hepaticae of west Greenland from ca. $66^{\circ} \mathrm{N}$ to $72^{\circ} \mathrm{N}$. - Meddelelser om Grønland 199 (1): 1-373.

SCHUSTER, R.M. \& N.A. KONSTANTINOVA. 1996. Studies on the distribution of critical arctic/subarctic Hepaticae with special reference to taxa found in Russia. - Lindbergia 21 (1): 26-48.

SCHUSTER, R.M., W.C. STEERE \& J.W. THOMSON. 1959. The terrestrial cryptogams of northern Ellesmere Island. - Bulletin of the $\mathrm{Na}$ tional Museum of Canada 164: 1-132.

SHAW, B., B. CRANDALL-STOTLER, J. VÁŇA, R.E. STOTLER, M.
VON KONRAT, J.J. ENGEL, C.E. DAVIS, D.G. LONG, P. SOVA \& A.J. SHAW. 2015. Phylogenetic relationships and morphological evolution in a major clade of leafy liverworts (phylum Marchantiophyta, order Jungermanniales): suborder Jungermanniineae. - Systematic Botany 40 (1): 27-45. http://dx.doi.org/10.1600/036364415X686314

SMITH, A.J.E. 1990. The liverworts of Britain and Ireland. - Cambridge University Press: Cambridge. $362 \mathrm{pp}$.

SÖDERSTRÖM, L. 1995. Preliminary Distribution maps of bryophytes in Norden. Vol. 1. Hepaticae and Anthocerotae. - Mossornas Vänner: Göteborg, Sweden. 55 pp.

SÖDERSTRÖM, L., E. URMI \& J. VÁŇA. 2002a. Distribution of Hepaticae and Anthocerotae in Europe and Macaronesia. - Lindbergia 27 (1): 3-47.

SÖDERSTRÖM, L., K. HASSEL, H. WEIBULL, A. ÂBOLIŇA, H.H. BLOM, K. DAMSHOLT, R. FAGERSTÉN, K.I. FLATBERG, A.A. FRISVOLL, M. HAAPASAARI, T. HALLINGBÄCK, L. HEDENÄS, E. HEEGAARD, S. HUTTUNEN, N. INGERPUU, P. ISOVIITA, B. JÓHANNSSON, I. JUKONIENË, T. KOPONEN \& J. LEWINSKYHAAPASAARI. 2002b. Preliminary Distribution maps of bryophytes in Northwestern Europe. Vol. 1. Hepaticae and Anthocerotae (2nd ed.). - Mossornas Vänner: Göteborg, Sweden. 55 pp.

SÖDERSTRÖM, L., E. URMI \& J. VÁŇA. 2007. The distribution of Hepaticae and Anthocerotae in Europe and Macaronesia - Update 1427. - Cryptogamie, Bryologie 28: 299-350.

SÖDERSTRÖM, L., A. HAGBORG, M. VON KONRAT \& M.A.M. RENNER. 2008. Early Land Plants Today: Liverwort Checklist of Checklists. - Fieldiana Botany 47: 105-130. http://dx.doi.org/10.3158/ 0015-0746-47.1.105

SÖDERSTRÖM, L., J. VÁŇA, A. HAGBORG \& M. VON KONRAT. 2013. Notes on Early Land Plants Today. 35. Notes on Lophoziaceae (Marchantiophyta) - Phytotaxa 97 (2): 27-35.

SOFRONOVA, E.V. \& A.D. POTEMKIN. 2018. Four rare liverwort species: distribution, ecology, taxonomy. - Novosti sistematiki nizshikh rastenii 52 (2): 505-518. http://dx.doi.org/10.31111/nsnr/ 2018.52.2.505

SOMMERFELDT, S.C. 1833. Bidrag til Spitsbergens og Beeren-Eilands flora, efter herbarier, medbragte afM. Keilhaue. - Magazin for Naturvidenskaberne 11 (2): 234-252.

SOTIAUX, A., O. SOTIAUX \& A. VANDERPOORTEN. 2006. Discovery of the rare liverwort Haplomitrium hookeri in Belgium: relictualism or long-distance dispersal? - Cryptogamie, Bryologie 27: 367373.

SPRUCE, R. 1882. On Cephalozia (a genus of Hepaticae) its subgenera and some allied genera. - Slater: Malton. $99 \mathrm{pp}$. http://dx.doi.org/ 10.5962/bhl.title.46289

STEBEL, A., R. OCHYRA, N.A. KONSTANTINOVA, W. ZIAJA, K. OSTAFIN \& W. MACIEJOWSKI. 2018. A contribution to the knowledge of bryophytes in polar areas subjected to rapid deglaciation: A case study from southeastern. - Acta Societatis Botanicorum Poloniae 87 (4:3603): 1-26. http://dx.doi.org/10.5586/asbp.3603

STEERE, W.C. 1953. On the geographical distribution of arctic bryophytes. - Stanford University Publications. Biological Sciences 11: $30-47$.

STEERE, W.C. 1954. Bryophytes (in: The cryptogamic flora of the Arctic). - Botanical Review. Lancaster 20 (6/7): 425-450.

STEERE, W.C. \& H. INOUE. 1978. The Hepaticae of arctic Alaska. Journal of the Hattori Botanical Laboratory 44: 251-345.

STEPHANI, F. 1898. Species hepaticarum 1.-Bulletin de l'Herbier Boissier 6 (10): 757-799. http://dx.doi.org/10.5962/bhl.title.95494

STEPHANI, F. 1901a. Species hepaticarum 2. - Bulletin de l'Herbier Boissier (sér. 2) 1 (2): 140-177. http://dx.doi.org/10.5962/ bhl.title.95494

STEPHANI, F. 1901b. Species hepaticarum 2. - Bulletin de l'Herbier Boissier (sér. 2) 1 (5): 477-521. http://dx.doi.org/10.5962/ bhl.title.95494 
STEPHANI, F. 1902. Species hepaticarum 2. - Bulletin de l'Herbier Boissier (sér. 2) 2 (2): 157-179. http://dx.doi.org/10.5962/bhl.title.95494 STEPHANI, F. 1910. Species hepaticarum 4. - George \& Cie: Genčve \& Bale. 448 pp. http://dx.doi.org/10.5962/bhl.title.95494

STOTLER, R.E. \& B. CRANDALL-STOTLER. 2017. A synopsis of the liverwort flora of North America north of Mexico. - Annals of the Missouri Botanical Garden 102 (4): 574-709. http://dx.doi.org/10.3417/ 2016027

SUMMERHAYES, V.S. \& C.S. ELTON. 1923a. Contribution to the ecology of Spitsbergen and Bear Island. - Journal of Ecology 11 (2): 214-286.

SUMMERHAYES, V.S. \& C.S. ELTON. 1923b. Contribution to the ecology of Spitsbergen and Bear Island. III. Spitsbergen. - Journal of Ecology 11 (2): 233-282. http://dx.doi.org/10.2307/2255865

ŚWIĘS, F. \& K. KARCZMARZ. 1991a. Bryophytes collected in arctic tundra of Chamberlin region (Western Spitsbergen) in 1987 and 1988. - Annales Universitatis Mariae Curie-Skłodowska, C Biologia 46: 29-43.

ŚWIĘS, F. \& K. KARCZMARZ. 1991b. Bryophytes collected in arctic tundra of the Logne region (Western Spitsbergen) in 1988. - Wyprawy Geograficzne na Spitsbergen 1991: 145-162.

ŚWIĘS, F. \& K. KARCZMARZ. 1993a. Bryophytes collected in the arctic tundra of Calypsostranda region (western Spitsbergen) in 1987 and 1988. - Annales Universitatis Mariae Curie-Skłodowska, C Biologia 48: 49-66.

ŚWIĘS, F. \& K. KARCZMARZ. 1993b. Bryophytes collected in arctic tundra of the Lyellstranda region (Western Spitsbergen) in 1987 and 1988 - In: Repelewska-Pękalowa, J. \& K. Pękala, Eds. Polar session. Arctic environment research. Lublin, Poland. Wyprawy Geograficzne na Spitsbergen. Lublin: Maria Curie-Sklodowska Univ., pp. 249-271.

THINGSGAARD, K. \& K. DAMSHOLT. 2007. Bryological notes from Svalbard. - Lindbergia 31 (3): 126-130.

UNAN, A.D., A.D. POTEMKIN, S. URSAVAŞ, S. ÇALIŞKAN \& M. ÖREN. 2021. New records of two Scapania species (Scapaniaceae, Marchantiophyta) from north of Turkey. - Plant Biosystems 155 (4): 679-684. http://dx.doi.org/10.1080/11263504.2020.1779836

VÁŇA, J. 1973. Studien über die Jungermannioideae (Hepaticae). 2. Jungermannia subg. Jungermannia. - Folia Geobotanica et Phytotaxonomica 8 (3): 255-309. http://dx.doi.org/10.1007/BF02852828

VÁŇA, J. 1974. Studien über die Jungermannioideae (Hepaticae). 6. Jungermannia subg. Solenostoma: Europäische und nordamerikanische Arten. - Folia Geobotanica et Phytotaxonomica 9 (4): 369 423. http://dx.doi.org/10.1007/BF02852451

VÁŇA, J. 1975. Studien über die Jungermannioideae (Hepaticae). 7. Jungermannia subg. Plectocolea: Europäische und nordamerikanische Arten. - Folia Geobotanica et Phytotaxonomica 10 (1): 67-99. http:/ /dx.doi.org/10.1007/BF02855102

VILNET, A.A., I.A. MILYUTINA, A.V.TROITSKY \& N.A. KONSTANTINOVA. 2005. On phylogeny and systematics of genus Lophozia (Dumort.) Dumort. s.str. based on trnL-trnF sequences of chloroplast DNA and ITS 1-2 sequences of nuclear DNA. in: Afonina, O.M., A.D. Potemkin \& I.V. Chernyadyeva, Eds. Actual problems in bryology: Proceedings of the international meeting devoted to the 90-th Anniversary of A. L. Abramova (Saint Petersburg, November 22-25, 2005). St. Petersburg: Komarov Botanical Institute, pp. 37-43.
VILNET, A.A., N.A. KONSTANTINOVA \& A.V. TROITSKY. 2010. Molecular insight on phylogeny and systematics of the Lophoziaceae, Scapaniaceae, Gymnomitriaceae and Jungermanniaceae. - Arctoa 19: 31-50. http://dx.doi.org/10.15298/arctoa.19.02

VILNET, A.A., N.A. KONSTANTINOVA \& A.V. TROITSKY. 2011. Taxonomical rearrangements of Solenostomataceae (Marchantiophyta) with description of a new family Endogemmataceae based on trnLF cpDNA analysis. - Folia Cryptogamica Estonica 48: 125-133.

VILNET, A.A., N.A. KONSTANTINOVA \& A.V. TROITSKY. 2012a. Molecular phylogeny and systematics of the suborder Cephaloziineae with special attention to the family Cephaloziaceae s.l. (Jungermanniales, Marchantiophyta). - Arctoa 21: 113-132. http://dx.doi.org/ 10.15298/arctoa.21.11

VILNET, A.A., N.A. KONSTANTINOVA \& A.V. TROITSKY. 2012 b. Molecular phylogenetic data on reticulate evolution in the genus Barbilophozia Löske (Anastrophyllaceae, Marchantiophyta) and evidence of non-concerted evolution of rDNA in Barbilophozia rubescens allopolyploid. - Phytotaxa 49: 6-22.

VIRTANEN, R., P.A. LUNDBERG, J. MOEN \& L. OKSANEN. 1997. Topographic and altitudinal patterns in plant communities on European arctic islands. - Polar Biol. 17 (2): 95-113. https://doi.org/ 10.1007/ s003000050111

VITT, D.H., D.G. HORTON \& J. PICKARD. 1987. An annotated list and the phytogeography of the bryophytes of Keele Peak, Yukon - an isolated granitic mountain. - Memoirs of the New York Botanical Garden 45: 198-210.

VON KONRAT, M., L. SÖDERSTRÖM \& A. HAGBORG. 2010. The Early Land Plants Today project: A community-driven effort and a new partnership with Phytotaxa. - Phytotaxa 9 (1): 11-21. DOI: https:// doi.org/10.11646/phytotaxa.9.1.4

WATSON, W. 1922. Spitsbergen liverworts. - Journal of Botany, British and Foreign 60: 327-330.

WEGENER, C., M. HANSEN \& L.B. JACOBSEN. 1992. Vegetasjonsovervåking på Svalbard 1991. Effekter av reinbeite ved Kongsfjorden, Svalbard. - Norsk Polarinstitutt meddelelser 121: 1-53.

WIETRZYK, P., M. WĘGRZYN \& M. LISOWSKA. 2016. Vegetation diversity and selected abiotic factors influencing the primary succession process on the foreland of Gåsbreen, Svalbard. - Polish Polar Research 37 (4): 493-509. http://dx.doi.org/10.1515/popore-20160026

WORLEY, I.A. 1969. Haplomitrium hookeri from western North America.-Bryologist 72 (2): 225-227. http://dx.doi.org/10.2307/3241673

WORLEY, I.A. 1972. The bryo-geography of southeastern Alaska. - University of British Columbia: Vancouver. $715 \mathrm{pp}$.

WULFF, T. 1902. Botanische Beobachtungen aus Spitzbergen. - E. Malmström: Lund. $116 \mathrm{pp}$.

XIANG, Y.-L., L. SHU \& R.-L. ZHU. 2016. Marchantia longii (Marchantiaceae), a new species from northwestern Yunnan, China. - Bryologist 119 (3): 280-289. http://dx.doi.org/10.1639/0007-2745-119.3.280

ZHANG, W.P., L. SHU \& R.-L. ZHU. 2017. Scapania paraphyllia, a new synonym of Scapania koponenii (Marchantiophyta, Scapaniaceae) with special reference to paraphyllia, pseudoparaphyllia, and paraphyses. - Journal of Bryology 39 (3): 277-284. http://dx.doi.org/ 10.1080/03736687.2017.1281866

Received 12 September 2021

Accepted 15 November 2021 